\title{
Queer Youth and Everyday Spaces in Wellington, Aotearoa New Zealand: A Photovoice Project
}

\section{Matthew Barry Scott}

A thesis submitted to Victoria University of Wellington in partial fulfilment of the requirements for the degree of

\section{Master of Science in Geography}

School of Geography, Environment and Earth Sciences

Victoria University of Wellington

Te Whare Wānanga o te Ūpoko o te Ika a Māui 


\section{Abstract}

Queer people are often 'othered' in everyday spaces because of the assumptions, practices, and beliefs that reinforce heterosexuality and cis-gendered bodies as normal and natural. For queer youth, these experiences are further exacerbated by their age and agency. Yet there has been little explicit geographic scholarship focused on understanding how queer youth navigate heteronormativity and practice their subjectivities in different everyday spaces.

In this thesis, I draw on the work of queer theory and geographies of sexuality literature to consider how subjectivities are constructed, controlled, and experienced by some queer youth in everyday spaces of Wellington, Aotearoa-New Zealand. I work within a transformative epistemology, and use photovoice as my research method to present the reflective engagements of six queer youth aged between sixteen and twenty-two through their photographs and accompanying narratives. Using Foucauldian discourse analysis to examine the participants' visual and verbal texts yields contradicting and varying experiences of heteronormativity.

Processes of subjectivity negotiation, queering space, conforming strategies, and gender performance influence how queer youth are placed within their everyday spaces. Safe spaces, like nature, the stage, and queer youth groups provide queer youth with the ability to be self-expressive, escape from the pressures of everyday life, and be surrounded with other queer people. Such spaces can be enhanced through activism, queer representation, and with the presence of animals and friends. This research contributes academically to research within geographies of sexualities and works towards disseminating these findings through a collaborative zine to support efforts to counter some of the dominating effects of heteronormativity identified by the queer youth.

\section{Key words:}

queer, queer youth, heteronormativity, subjectivity, space, place, geographies of sexuality, queer geography, queer theory, photovoice, New Zealand 


\section{Acknowledgments}

I would firstly like to thank the participants that contributed their time and energy into the photovoice project. The opportunity to get to know such honest, lovely, and happy people has been the biggest highlight in this research journey. Without you, this thesis would not have been possible.

To my supervisor Associate Professor Sara Kindon, it has been both a pleasure and honour to work with you through this journey and through journeys past. Your words of encouragement, scholarly knowledge, and unwavering support of me has made this thesis what it is today. I feel extremely privileged to have had you as my supervisor and I look forward to our paths crossing in the future.

To my fellow Masters students - especially Janie Walker, Lisa Dowling, and Sky Halford - I thank you for the shared panic and stress, but also the joy and comradery of going through this together. I am also grateful to my friends for excusing my absence from their lives while putting this thesis together.

To my family, I thank you for your support and love during this past year. And to Josh, who has endured me at my best and at my worst - thank you. 


\section{Table of Contents}

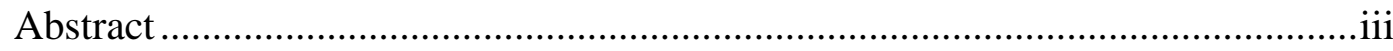

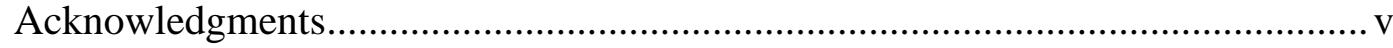

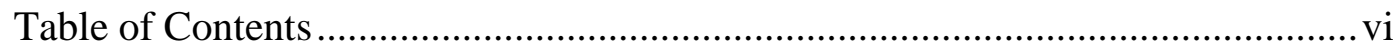

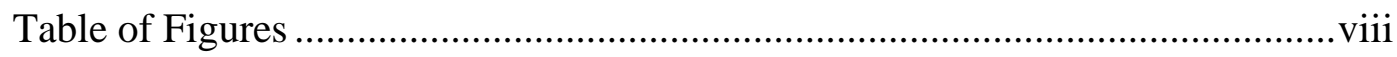

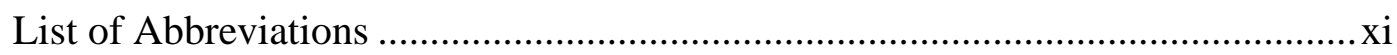

CHAPTER ONE: INTRODUCTION ................................................................... 1

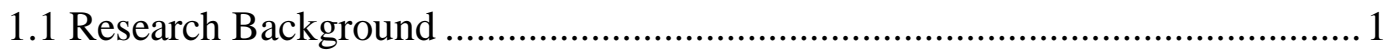

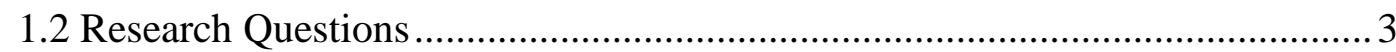

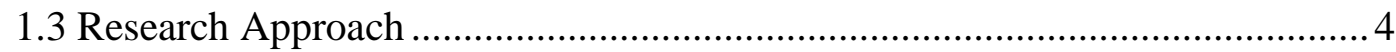

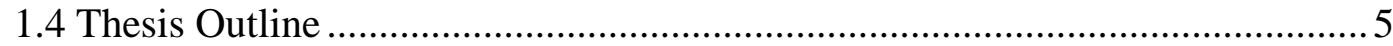

CHAPTER TWO: THEORETICAL FRAMEWORK ....................................... 7

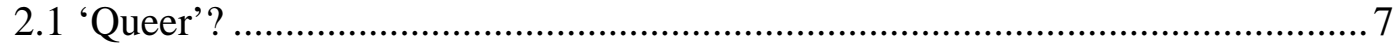

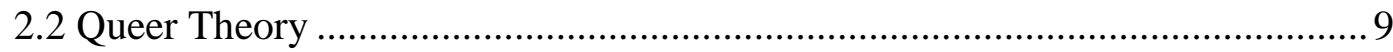

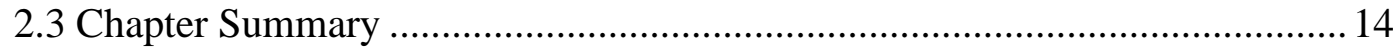

CHAPTER THREE: QUEER GEOGRAPHIES............................................ 15

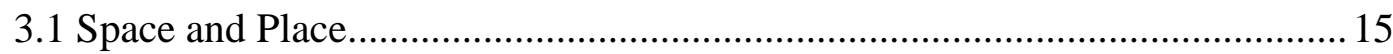

3.2 Gender, Sexuality, and Queerness in Geography ........................................20

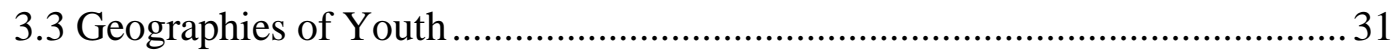

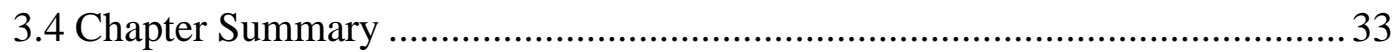

CHAPTER FOUR: METHODOLOGY ................................................................... 35

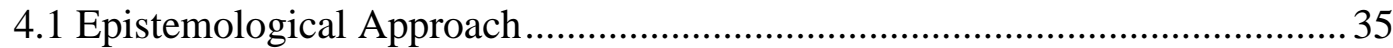

4.2 Queer, Multi-Sensory, and ‘Everyday’ Methodology ......................................35

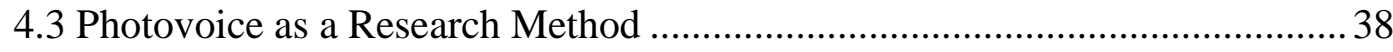

4.4 Ethics, Positionality, and Representation....................................................... 51

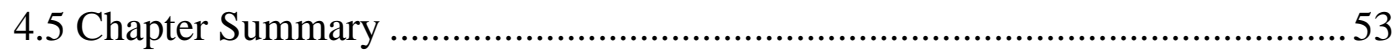




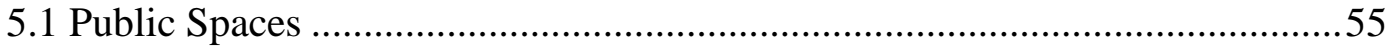

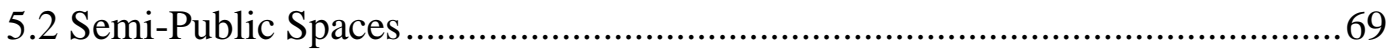

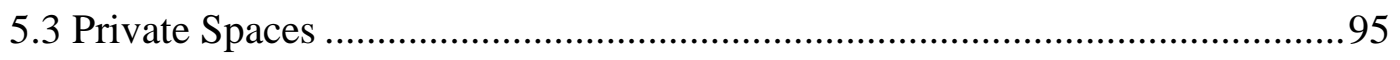

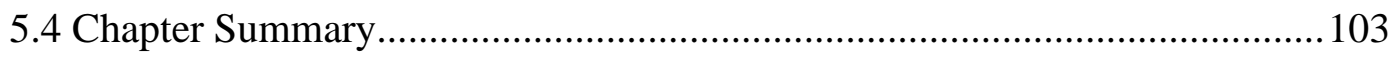

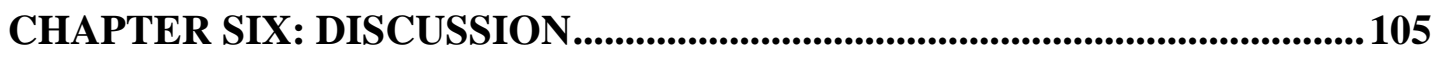

6.1 Heteronormativity in Everyday Spaces..................................................... 105

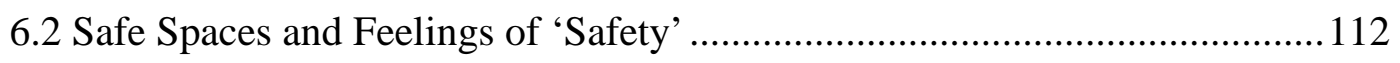

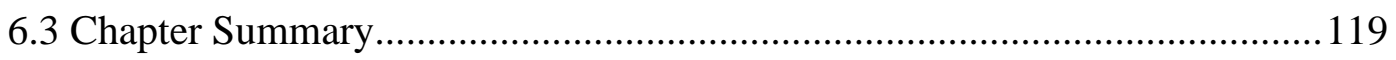

CHAPTER SEVEN: CONCLUSION AND FUTURE RESEARCH ..................121

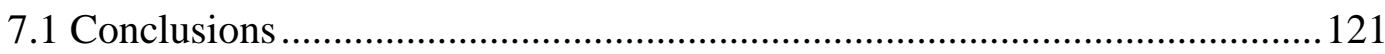

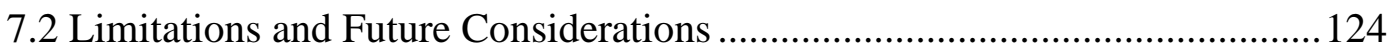

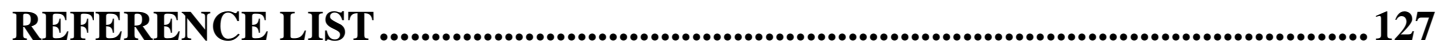

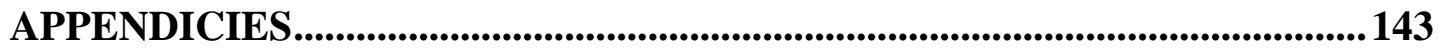

Appendix A - Photo Information Sheet........................................................... 143

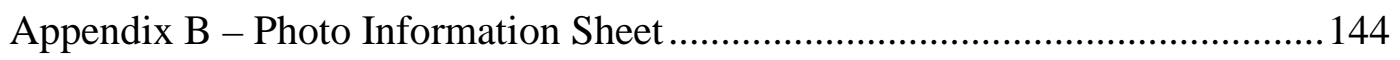

Appendix C - Health and Safety Information Sheet........................................145

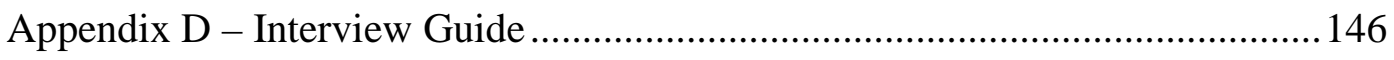

Appendix E - Participant Information Sheet .................................................148

Appendix F - Participant Consent Form....................................................... 151 


\section{Table of Figures}

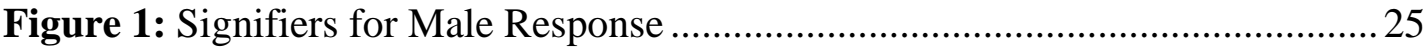

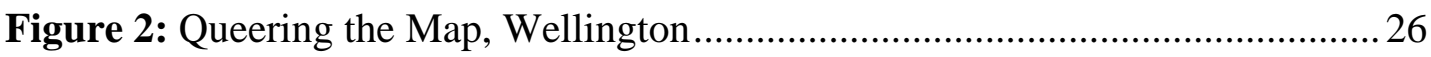

Figure 3: Focus Group Two at Toi Poneke Arts Centre ......................................... 43

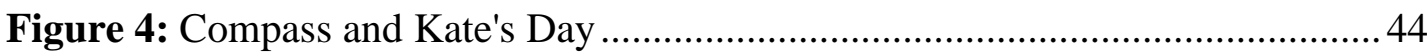

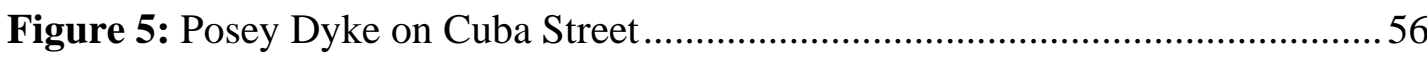

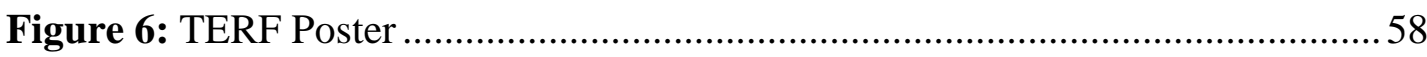

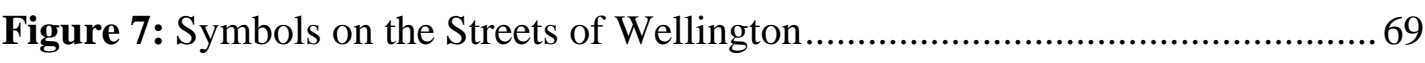

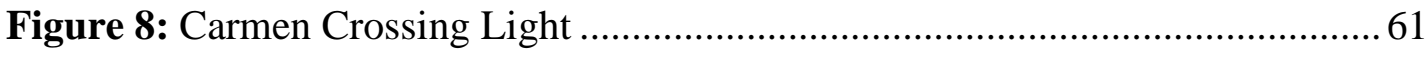

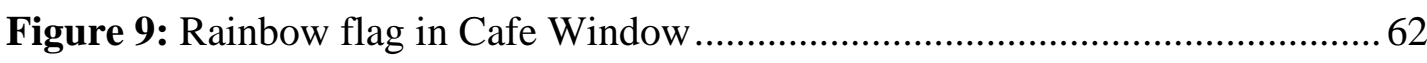

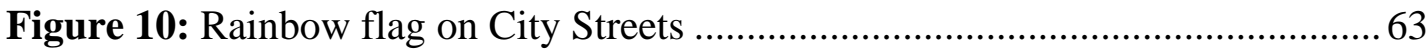

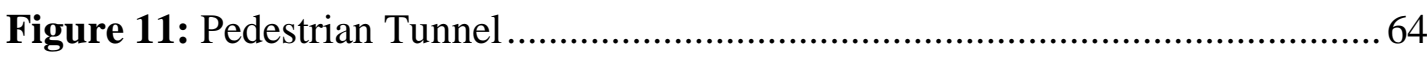

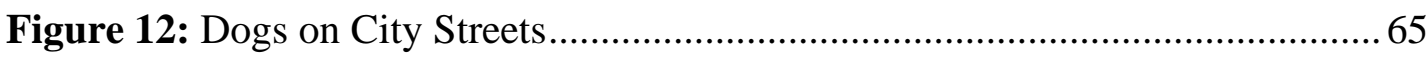

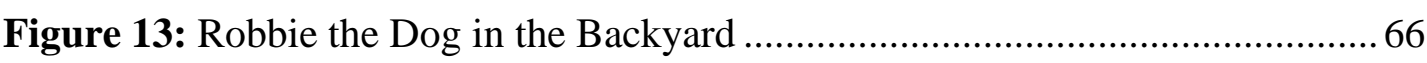

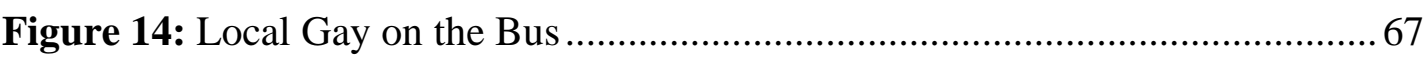

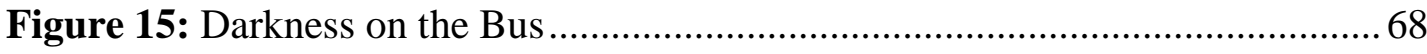

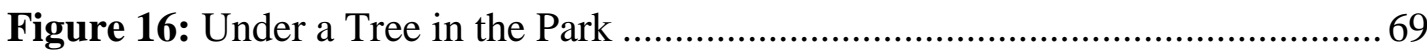

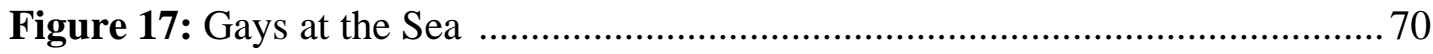

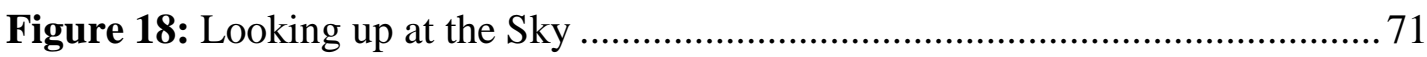

Figure 19: Taking the Time to Appreciate Nature................................................ 71

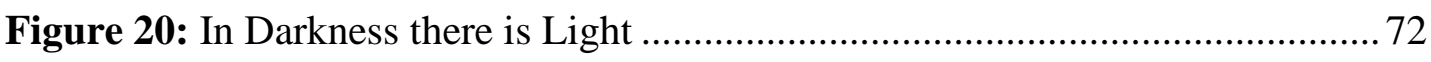

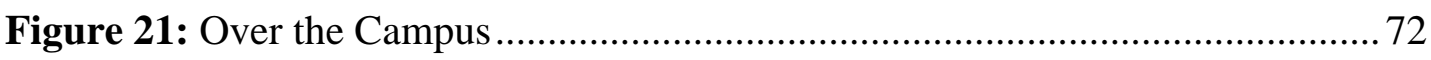

Figure 22: From Behind the Drag Curtain ............................................................ 74

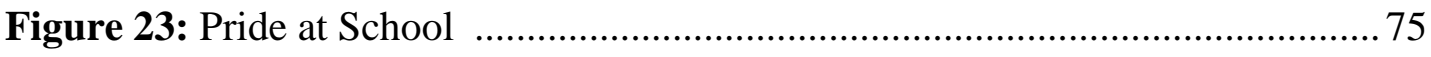

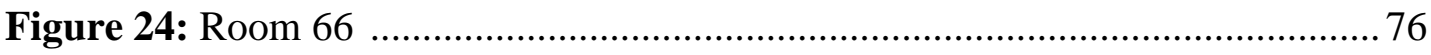

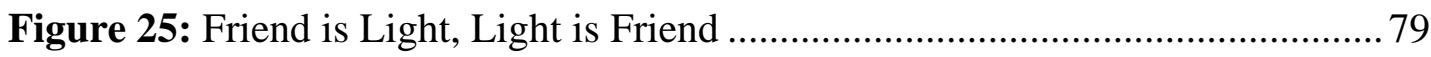

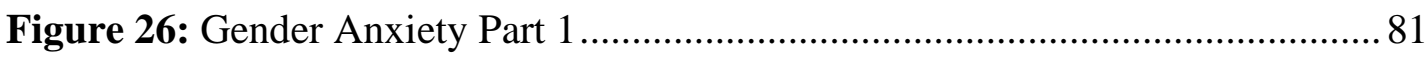

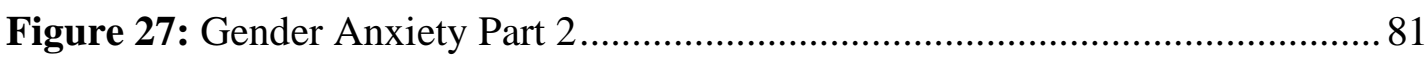

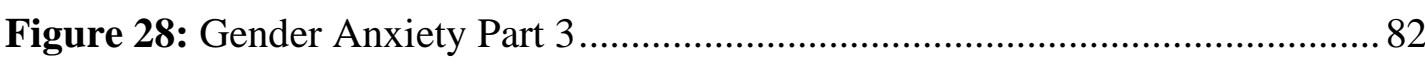

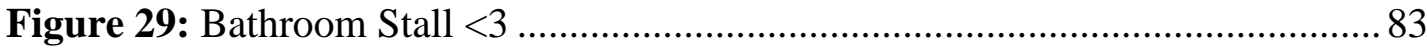

Figure 30: The Stage is Sacred, So are You ......................................................... 86

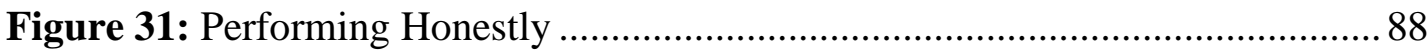




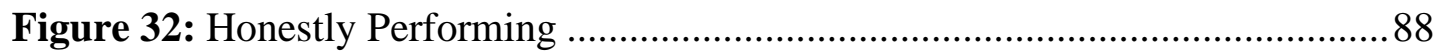

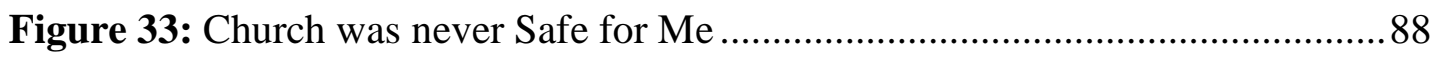

Figure 34: Church was never Safe for Me Part 2...................................................90

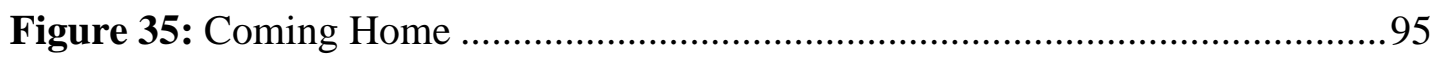

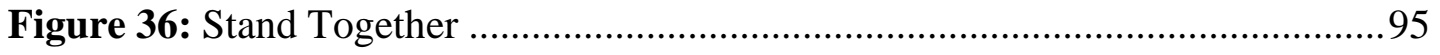

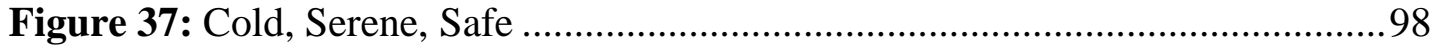

Figure 38: The Bed is Where I Hang out a lot with my Partner. .............................99

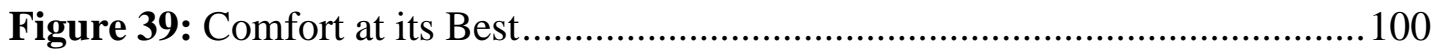

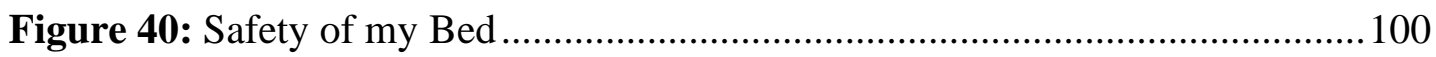

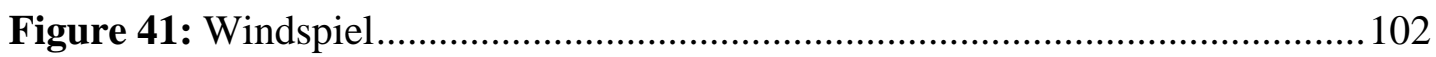

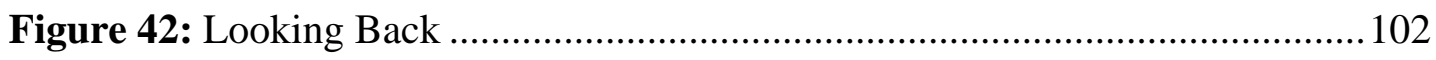

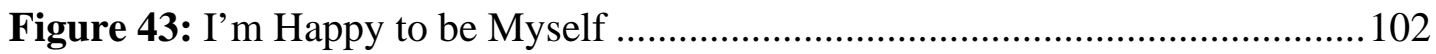




\section{List of Abbreviations}

Cis-Het Cis-Gender Heterosexual

FAB Female Assigned at Birth

LGBT Lesbian, Gay, Bi-sexual, and Transgender

PAR Participatory Action Research

QSA Queer Straight Alliances

TERF Trans Exclusionary Radical Feminist 


\section{CHAPTER ONE: INTRODUCTION}

\subsection{Research Background}

It was not until my partner and I were called faggots that I realised that a big part of my life was not 'normal'. It is a weird feeling to be singled out on a city street when choosing to show affection and connection to the person one loves through the simple act of holding hands. Ever since then however, when in public, my boyfriend and I limit our hand holding and other expressions of love so as to avoid further verbal or physical attack.

Incidents like these are very common for people who do not identify as cis-gender ${ }^{1}$ or heterosexual. One's chances at life as a queer person ${ }^{2}$ are heavily dependent on where one is born, the political climate, where one works, and the people with whom one surrounds oneself. For some of us queers, our sexuality and gender expression is still a matter of life or death: Being queer is punishable by death in eight United Nations member states, a person can be imprisoned for same-sex acts in 72, and 19 have 'morality' laws that outlaw public promotion of same-sex expression (Carroll \& Mendos, 2017).

For queer youth, there are also age-related disparities compared to their cis-gendered, heterosexual peers. Around the world, queer youth face issues with homelessness, discrimination perpetuated by family members, abuse in the schooling system, and restricted access to health resources. In the United States of America, 70 percent of queer youth have been bullied at school because of their sexual orientation, while 48 percent of queer youth who are out to their parents say that their families make them feel bad for their queer identity (Human Rights Campaign, 2018). In developing states, most do not have non-discrimination statutes regarding queer individuals' access to housing, government services, and medical assistance, which are inherited from often colonial era laws (Advocates for Youth, 2016).

\footnotetext{
${ }^{1}$ Cis-gender refers to a gender subjectivity, or performance in a gender role, that society deems to match the person's assigned sex at birth.

2 The term queer is used through-out this thesis to refer to sexual and gender identities that do not fit into the normalised binary categories of male/female and hetero-/homo-sexual. I discuss the term in more detail in Chapter 2.1.
} 
In Aotearoa-New Zealand, queer youth are more likely to be bullied, be physically harmed, and be afraid of violence at school. 59.4 percent have attempted self-harm, 40 percent of transgendered youth have been unable to access appropriate health care, 41.3 percent of queer youth express depressive symptoms, and worryingly, this percentage of queer youth is increasing (Lucassen, Clark, Moselen, \& Robinson, 2014).

But it is not all doom and gloom: As of 2017, 24 states recognise same-sex marriage, 26 allow joint child adoption, and 85 have different forms of civil protection towards sexuality and gender personhood which are imbedded in constitutions, employment, and hate crime laws (Carroll \& Mendos, 2017). In New Zealand, the Marriage (Definition of Marriage) Amendment Act 2013 was passed in the House of Representatives to allow same-sex couples to legally marry (previously same-sex couples were only allowed to be in civil partnerships) and historic homosexual convictions for sodomy and 'unnatural offences' were recently expunged. For queer youth, national organisations like InsideOUT and RainbowYOUTH are actively involved in improving targeted support in schools and the removal of caps to the number of gender reassignment surgeries performed each year (Coughlan, 2018).

That said, there is a long way to go before queer subjectivities ${ }^{3}$ and expressions of affection in public spaces are normalised. Geographies of sexuality and queer geography literature, which attends to the construction of binaries like male/female and heterosexual/homosexual and how these organise themselves in different spaces and places, are two academic disciplines working towards understanding how queer subjectivities and expressions can be normalised. Currently, the activities of people like myself and my partner are controlled by political and spatial discourses that normalise straight and cis-gendered bodies within our society. The normalisation and dominance of heterosexuality means that a lot of our existence is seen as 'out-of-place' and thus our realities reflect inequalities of power, access, and belonging.

These inequalities are not uncommon, and occur in our everyday spaces with which we interact. Homes, city streets, cafes, bars, bathrooms, and workplaces have all been coded to heterosexual and cis-gendered values. Overlooked or invisible in the everyday because of their seemingly natural and normalised dominance, these values

\footnotetext{
${ }^{3}$ I use to the term subjectivity rather than identity in this thesis. I detail its use in Chapter 2.2.2.
} 
impact everyone. But it is how heteronormativity in everyday spaces plays out and to what effect for queer, young bodies that is of central concern to me in this thesis.

At a personal level, this concern arises from my own lived experiences and my desire to learn more because of my relatively short time being out and active within the queer community. I am still young and learning about what it means to be 'out' in this country. At an intellectual level, this concern reflects wider debates in human geography about how space, gender, and sexuality are mutually constituted and investigates the integration of this in everyday life for queer youth. And at a political level, I want to use this thesis as a vehicle to honour, enhance, and promote participants' voices within a wider initiative to highlight powerful discursive structures and their effect on queer youth. As such, this thesis explores the experiences of other self-identified queer youth within the Wellington region of New Zealand as they interact with their everyday spaces and places to understand how this comes to affect their subjectivity.

\subsection{Research Questions}

With sparse research in geographies of sexuality and queer geography into the experiences of queer youth and their interactions with space and place, this research uses Judith Butler's theory on performativity, Michel Foucault's ideas around the inspecting gaze, and discussions by Gill Valentine on conforming strategies, to pay particular attention to how some Wellington queer youth perform, alter, supress, conform, confront, and engage with their subjectivities in everyday and familiar spaces. It also enquires into how queer youth create 'safe' space in light of heteronormative structures. Specifically I ask:

1. What are some of Wellington queer youths' experiences of their subjectivities in everyday spaces?

2. How does heteronormativity inform these experiences and the performance of subjectivities?

3. What aspects of everyday spaces make them 'safe' for Wellington queer youth, and how can these aspects be enhanced to counter heteronormativity?

In answering the first question, this thesis presents photographs and discussions with six queer youth from the Wellington region about their experiences with their everyday and commonly visited spaces. For my second research question, I investigate 
how heteronormativity specifically impacts on these queer youth's experiences and how some queer youths' subjectivities are performed. For my third research question, I explore what makes space 'safe' for self-identified queer youth, and discuss the ways in which heteronormativity can be challenged to enable spaces that provide inclusion and equity, while also improving the right for freedom of expression for queer youth. Through responding to these questions, I contribute to the growing geography of sexualities sub-discipline and raise awareness to the experiences of queer youth in their everyday space.

\subsection{Research Approach}

To answer these research questions and explore the experiences of queer youths' subjectivities in everyday spaces, a transformative approach to research is implemented. A transformative paradigm provides the impetus for my research aim to raise awareness and promote more tolerance of the queer community, help foster positive queer experiences of space and place, and highlight the influence of heteronormativity in society in general. In doing so, I hope to increase the range of ‘safe’ spaces, equitable access, and belonging for queer youth.

My engagement within queer theory and geographies of sexualities provides the theoretical framework in which to place my research. Key theorists and scholars are analysed to build an understanding of the prior research conducted on space, gender, and sexuality, and also guide the research to focus on discursive structures, binaries, and performative views on gender and sexuality. It also highlights the lack of research focused on the experiences of queer youth and their everyday space.

Participatory action research (PAR) principles and ethical considerations have guided the research from start to finish. Participants were actively engaged in the research process to enable insightful representation all while increasing the capability of the participants and the wider queer community to increase their own skills and knowledge through personal reflections (Kindon, Pain, \& Kesby, 2007).

These research paradigms provided the foundations for a qualitative methodology which implemented various methods including in-depth interviews, focus groups, photovoice, and collaborative zine design with a purposeful sample of Wellington queer youth. 'Youth' for this project was defined as between the ages of 12 and 24 
years, aligning with definition used by InsideOUT, my partner organisation, and Te Manatū Whakahiato Taiohi, Ministry of Youth Development.

Foucauldian discourse analysis provided a framework to analyse the data collected to highlight the discursive structures that enable and exclude access and feelings of safety in everyday spaces of the queer youth.

\subsection{Thesis Outline}

My thesis has begun with a brief introduction into the positioning of this research within the geographies of sexuality and queer theory literature by highlighting my research questions and transformative aim. It has also presented an introduction into my research approach.

In chapter two, I review queer theory and the work of theorists including Judith Butler and Michel Foucault to begin to frame my thesis and its inquiry. I also discuss the key concepts of heteronormativity, subjectivity, and identity.

Chapter three presents different discussions on the spatial politics of sexuality and gender. To begin, I introduce geographies of sexuality and queer geography disciplines and highlight prominent works that will be used to analyse my results. I engage with ideas of space and place, Western society's dichotomy of private and public spaces, queer space, conforming strategies, and conclude with a brief look at where queer youth are placed within these.

In chapter four, I discuss my research approach, introduce my participants, outline the methods I adopted, along with the Foucauldian discourse analysis strategies used to analyse my participants' photographs and transcripts. Here, I also reflect on my positionality and how this influenced my understanding and representation of the queer participants’ experiences.

In chapter five, I attend to my first research question and present the participants' photographs and their accompanying captions. This chapter and the photographs are divided into three sections - public spaces, semi-public spaces, and private spaces.

Chapter six is dedicated to the analysis and discussion of the participants' photographs in relation to the remaining research questions. 
Chapter seven concludes the research by providing an overview of the photovoice project. I revisit the research questions to distil the key findings from the previous chapters, and then go onto covering the limitations of this research, I finish with discussing future directions for geographic research relating to my research, its limitations, and its findings. 


\section{CHAPTER TWO: THEORETICAL FRAMEWORK}

In chapter two I present the history of the term queer and detail its 'identity', as well as its political and theoretical applications. I then go on to discuss queer theory and how this has informed the arguments presented in this thesis. I end by defining key ideas of heteronormativity and subjectivity which will inform subsequent analysis of participants' photographs and narratives.

\section{1 'Queer'?}

The definition of queer has been rearticulated and recirculated over time and has had varying characteristics and developments that are linked to defiance, struggle, celebration, and reclamation (Giffney, 2009). Even to this day, the act of defining the term leads to more questions than answers. The opacity in its definition encourages multiple meanings about queer as a political movement, an identity category, and a theoretical approach to academic inquiry (Giffney, 2009).

Historically, the term was predominately used as a slur to refer to gay men as 'different' or 'unusual'. More recently, the word has been powerfully reclaimed to signal a counter-offensive to homophobia and oppression relating to aspects of gender and sexuality (Giffney, 2009). Central to activism associated with: The Stonewall riots in 1969 New York; the AIDS crisis that was affecting queer communities (predominantly the gay community) across the globe; and the rise of anti-gay legislation brought in by governments like the Thatcher government in the United Kingdom (Johnston \& Longhurst, 2010), geographers David Bell and Gill Valentine (1995, p. 20) explain that:

... queer has become a notoriously indefinable watchword for certain so-called radical theoretical and activist stances, ... the first of which was arguably the act of reclaiming the very word 'queer' from its use as homophobic slang to be a label used by a variety of sexual dissidents.

Judith Butler (1993, p. 226) also says that the reclamation of the word queer "derives its force precisely through the repeated invocation by which it has become linked to accusation, pathologization, insult”. The overall reclamation of the term is not seen as an attempt to remove the negative conations and assimilate it back into wider society, 
but rather to challenge and contest the naturalness of heterosexuality within which the term 'queer' was produced to begin with (Browne, 2009). This is why we can now look at queer beyond its common function as a synonym and a short-hand umbrella term for sexual and gender dissidents (like identities within the lesbian, gay, bisexual, and transgender (LGBT) label).

As a theoretical approach, queer allows for a point of convergence of a multitude of different subjects (Jagose, 1996), creates a sense of what Cherry Smyth (1992) says is a potential for radical pluralism, and extends to a position that defines itself against whatever is constituted as 'normal' rather than just the heterosexual (Warner, 1993). Queer recognises identity as historically-contingent and socially-constructed, allows fluidity in identity recognising that sex, sexual, and gender identities are often never once-defined, and that persons cannot be placed discretely in one static 'identity' character (Giffney, 2009). In using the term queer, one champions a political persuasion to resist hegemonic discourses, exclusionary practices, codes of behavioural obedience, and identifications set by the dominant cultural force in any given society; particularly binary oppositions such as the heterosexual/homosexual, gender/sex, and man/woman (Johnston \& Longhurst, 2010).

There are criticisms however of the use of the term queer. Butler (2008) says "I worry when 'queer' becomes an identity. It was never an identity. It was always a critique of identity. I think if it ceases to be a critique of identity, it’s lost its critical edge” (p. 32, as quoted in Giffney, 2009, p. 4). Knopp (2007) also believes that the use of the term queer could impose homogeneity on what are arguably very different groups of people.

That said, if as Spargo (1999, p. 40) notes, “queer culture has reclaimed ‘queer’ as an adjective that contrasts with the relative respectability of 'gay' and 'lesbian', then queer theory could be seen as mobilising 'queer' as a verb that unsettles assumptions about sexed and sexual being and doing”. It is in this vein that I choose to use the term queer throughout this thesis - as both a quality (adjective), and as an attribute (verb) (Dilley, 1999). It is used to honour the term most commonly used by my research participants to describe their collective identity, while also paying attention to heterogeneity and intersectionality by using participants' own sexual and gender identity terms and by acknowledging their age, ethnicity, religion, and culture. I also use the term queer for the power it gives in its application within queer theory. 


\subsection{Queer Theory}

In using queer as a verb, queer defines "sexuality as a product of social relations and thereby suggests the history of sexuality to be 'the history of the subject whose meaning and content are in a continual process of change”” (Penn, 1995, p. 26). At the heart of queer theory is understanding how those meanings have come to be, alongside the power that they hold (Dilley, 1999). According to Seidman (1995, as quoted in Dilley, 1999), queer theory is:

... less a matter of explaining the repression or expression of a homosexual minority than an analysis of the hetero/homosexual figure as a power/knowledge regime that shapes the ordering of desires, behaviors [sic], and social institutions, and social relations - in a word, the constitution of the self and societ [sic] (p. 460).

Patrick Dilley (1999, p. 459) says that with queer theory, "[it] is not a question of 'who is queer', but 'how is queer'; now so much 'why are they queer', but 'why are we saying they are queer?” The answer to this question is possible when one interrogates and critiques how discursive structures are produced by a "compulsively heterosexual society” (Seidman, 1995, as quoted in Dilley, 1999, p.459).

When working within queer theory in qualitative social research, one takes the position that:

... requires an ontological shift comprehensively resistant in its exceptions to dominant normativity. A queering of standpoint in social research is a vigorous challenge to that which has constrained what may be known, who may be the knower, and how knowledge has come to be generated and circulated, [and] queers participate in positioning themselves through both authoring and authorizing experience (Honeychurch, 1996, as quoted in Dilley, 1999, p.458).

Queer theory is utilized by a number of different disciplines, but as Dilley (1999, p. 462) believes, all have three main tenants:

1. examination of lives and experiences of those considered non-heterosexual;

2. juxtaposition of those lives/experiences with lives/experiences considered 'normal'; 
3. examination of how/why those lives and experiences are considered outside of the norm.

My engagement with queer theory in this thesis follows its use by human and queer geographers as they have drawn on thinkers such as Michel Foucault and Judith Butler to attend to these three tenants.

Interdisciplinary queer scholarship often involves debates about Foucault's work into sexuality, discourse, and power. His work shifted the focus of the construction of the subject to an analysis of discourse, intuitions, and powers that discipline and reinforce the understandings of gendered, sexual, and sexed bodies (Browne \& Nash, 2010).

Foucault's view of discourse centres on the production and circulation of knowledge (Waitt, 2010). He was interested in "how particular knowledge systems convince people about what exists in the world (meanings) and determine what they say (attitudes) and do (practices)” (Waitt, 2010, p. 218). Discourse therefore reflects a constructivist view of knowledge creation and its implications for people, places, and things. It demands that questions be asked about the way social realities become naturalised (Waitt, 2010).

Foucault was also able to detail an example of how discourse played out in regulation of the sexualised body in his book Discipline and Punish (1979) where he discussed the effect of power on the body and how it is often a target for "penal repression" (Valentine, 2001, p. 27). Foucault argued that the discourse of ideal behaviour, appearance, and subjectivity are maintained not through policing, physical violence, or coercion, but through self-surveillance and self-correction generated from the disciplining power of an inspecting gaze (Valentine, 2001). He used the example of the Panopticon prison design by Jeremy Bentham to explain how the fact of being constantly watched (or the possibility of being watched) means that most individuals develop self-disciplining behaviours, which conform to dominant expectations. He said:

There is no need for arms, physical violence, material constraints. Just a gaze, an inspecting gaze, a gaze which each individual under its weight will end by interiorising to the point that he [sic] is his [sic] own observer, each individual thus exercising this surveillance over, and against himself [sic] (Foucault, 1979, p. 155). 
The control, disciplining, and punishment of bodies created what Foucault called 'docile bodies', which conform to social and historical specific discourse about what normal presentations of self and behaviour are in a particular space. The dominance of these specific sets of norms is not achieved solely through higher forms of power but multiple “... processes of different origin and scattered location, regulating the most intimate and minute elements of the construction of space, time, desire, embodiment” (Foucault, 1979, p. 133). Foucault saw power as not a totalising system, but rather as engrained in all levels of society, from the micro-level of the home, body and work, to the highest structural institutions of our society (McDowell, 1995).

Valentine (2001) explains that Foucault's work has been influential in the social sciences and in geography. His connection between discourse and bodies means we now understand that different discourse regimes produce different bodies. More importantly for this research, Foucault revealed that the individual body is manipulated and controlled by an "endless circuit of power and knowledge" (Valentine, 2001, p. 28) connecting the everyday to power at wider and more dominating scales and forces.

Butler's work (1990) is also some of the most influential in aspects relating to the construction of 'gender' within queer theory. Butler's view on gender is that it is the "repeated stylization of the body, a set of repeated acts within a highly rigid frame that congeal over time to produce the appearance of substance, of a natural sort of being” (1990, p. 33). These repeated acts mean that gender is not simply an aspect of what one is, but it is something that one does, and does recurrently in interaction with others (West \& Zimmerman, 1990). Butler suggests that “acts, gestures, enactments, generally constructed, are performative in the sense that the essence or identity that they otherwise purport to express the fabrications manufactured and sustained through corporeal signs and other discursive means” (Butler, 1990, p. 136). Gender is therefore not a natural thing; men are not naturally masculine and women are not naturally feminine. Rather, the repeated acts of masculine and feminine by gendered bodies make it appear that way. Further, gendered bodies are a constructed social relation produced by discourse. Although having a 'weighty materiality and biology', gendered bodies are always existent in political, economic, cultural, and social realms (Johnston \& Longhurst, 2010). 
To understand how the ideal gender and sexual subject is recreated through performative actions and in response to the inspecting gaze, Browne (2009) says the term heteronormativity was developed.

\subsubsection{Heteronormativity}

Heteronormativity is a term used in discussions of gender and sexuality to describe the ways that societal institutions and forms of behaviours reinforce and produce the belief that heterosexuality is the norm and anything different is not normal, or even abnormal (Bell, 2009). David Bell (2009, p. 115) sees heteronormativity as "a powerful but often unmarked set of assumptions, practices, and beliefs that constantly reinforce the normalness and naturalness of heterosexuality”.

Heterosexuality - in relation to hetero-normativity - can be seen on the sexual level consisting of two opposite bodies (with opposite genitals, chromosomes, and genders) participating in sexual intercourse, romance, and attraction. This view on sexuality is of the dominant one in Western societies and is often explained as normal because of links to biology and primal instincts where there is a 'natural' need to reproduce (Valentine, 1993). Valentine explains that normal sex is "monogamous, procreative heterosexuality” and involves vaginal penetration by a penis. But Bell (2009) explains that heterosexuality is not just a sexual practice. Rather the term evokes what is referred to as a 'heterosexual matrix' by Butler (1990) and incorporates aspects of "gender, sex, and desire, and binds these together through rituals, assumptions, and sociospatial practices” (Bell, 2009, p. 115). Bell also explains that:

The condensation of this matrix is the heterosexual couple - perhaps most notably the married couple. This is a romantic, sexual, (potentially) reproductive, monogamous, lifelong union of two oppositely-sexed subjects, ritualized through religious or civil ceremony and supported by countless institutions (government, religion, family, etc.) and practices (displaying affection, having children, commemorating anniversaries, etc.) (p. 114).

When the term heteronormativity is used, the hetero- is therefore not only engaging with the sexual orientation aspect of heterosexuality, but the normalisation and prescription of heterosexual identities, practices, and institutions and the subsequent exclusion and prohibition of others (Bell, 2009, see also earlier work by Warner, 1993). The driving force of heteronormativity is to enable normalised sexuality and 
constrain a person who lives outside of the boundaries of 'normal'. Heteronormativity is frequently present in our everyday lives and has been studied in numerous ways in geography (Hubbard, 2008).

As a dominant discourse, heteronormativity pervades all aspects of our life and is one of the forces in which our bodies are situated (Probyn, 2003). However, our identity is never constituted to any one factor - rather we are subjected to a myriad of different identities at one time. Our subjectivity is situated in the nexus of gender and sexuality as well race, class, ability etc. And at each set of these subjectivities we are set with being at the end of a binary: man/women, old/young, straight/gay. I discuss subjectivity and its use over the term 'identity' in this thesis now.

\subsubsection{Subjectivity v. Identity}

The terms subjectivity and identity often get used interchangeably but there are differences in the two terms when used in a queer theoretical context:

... one's identity can be thought of as that particular set of traits, beliefs, and allegiances that, in short- or long-term ways, gives one a consistent personality and mode of social being, while subjectivity implies always a degree of thought and self-consciousness about identity, at the same time allowing a myriad of limitations and often unknowable, unavoidable constraints on our ability to fully comprehend identity. Subjectivity as a critical concept invites us to consider the question of how and from where identity arises, to what extent it is understandable, and to what degree it is something over which we have any measure of influence or control (Hall, 2004, p. 3).

Subjectivity presents itself as tension between our ability to choose an identity and one that is prescribed for us. But, as Donald Hall (2004, p. 1) wrote:

We are widely led to believe that we have the freedom and ability to create and re-create our "selves" at will, if we have the will, but at the same time are presented with a suspiciously narrow range of options and avenues that will allow us to fit comfortably into society and our particular gendered, regional, ethnic, sexual subset of it. 
Our ability to determine what our identity is, is constrained by forces - like heteronormativity - that we often do not see. One is therefore forced, and subjected to, an identity from a prescribed list that one had no hand in writing (Hall, 2004).

The term subjectivity is therefore related to the ways that discourse and ideology and in the case of this thesis, discursive powers, are enacted on a 'subject'. The view of the subject can be many things at once:

First, the subject is a subject to itself, an "I", however difficult or even impossible it may be for others to understand this "I" from its own viewpoint, within its own experience. Simultaneously, the subject is a subject to, and of, others; in fact, it is often an "Other" to others, which also affects its sense of its own subjectivity ... Third, the subject is also a subject of knowledge, most familiarly perhaps of the discourse of social institutions that circumscribe its terms of being. Fourth, the subject is a body that is separate (except in the case of pregnant women) from other human bodies; and the body, and therefore the subject, is closely dependent upon its physical environment (Gagnier, 1991, p. 8).

Reflecting on these ideas, I choose to use the term subjectivity rather than identity. However, I made an informed decision to refrain from using the term subjectivity when working with the queer youth participants in the research process. This decision is based on the observation that participants commonly referred to their queerness as an 'identity' and it may have been too difficult to enforce the term subjectivity and its nuances on the participants in the photovoice project.

\subsection{Chapter Summary}

In this chapter, I have outlined the theoretical framework I have used to structure my approach to this research. I began with detailing the historical developments of the term queer and recognised its use as a quality and an adjective in this thesis. Next, I introduced how queer theory and its focus on understanding exceptions to dominant normativity will be an integral way of understanding and analysing my research. I have also shown the ways that queer theorists understand discourse, and the effect this has on the performance of gender and sexuality. Lastly, I have presented how gender and sexuality constitute subjects through heteronormative discourse and regulation. 


\section{CHAPTER THREE: QUEER GEOGRAPHIES}

Chapter three begins by presenting the key geographic terms of 'space' and 'place' and discusses the spatial politics of queer interactions within different spaces and places. I then move onto providing an overview of queer geography and geographies of sexuality from their earliest developments to current trajectories. Finally, the chapter looks at the development of youth and children's geographies and presents the argument for the need to start talking about queer youth geographies in more detail.

\subsection{Space and Place}

'Space' and 'place' are prominent ideas within the history of geographic thought, but there are numerous ways geographers conceptualise them. This section will highlight the diverse ways geographers have conceptualised space and place over different time periods and presents approaches from numerous key geographic thinkers that have influenced this thesis and its findings.

\subsubsection{The History of Spatial Thinking in Geography}

With developments to the discipline of geography there have been shifts in the way one conceptualises space. Phil Hubbard (2005, p. 41) explains that the use of the term 'space' provides human geographers with "the basis for a discipline that insists on grounding analyses of social and cultural life in appropriate geographic contexts”, but there are numerous ways geographers can approach understanding 'space'.

Geographic understanding of the late nineteenth and early twentieth centuries perceived space as merely a straightforward object that could be empirically measured, tested, and mapped (Hubbard, Kitchin, \& Valentine, 2004). It was thought that space was a blank container waiting to be filled by human activity, where its existence was not dependent on human interaction, but was rather a 'backdrop' to human lives (Hubbard et al., 2004). Geographers were then interested in the exploration and identification of the earth's surface in different ways with advances in mathematical and graphing techniques (Valentine, 2002).

In the 1970s, new approaches to understanding space arose. Geography saw the influence of behavioural and phenomenological theories (see Yi-Fu Tan), as well as structural and radical approaches such as Marxism (see David Harvey) and feminism 
(see Doreen Massey). Geographers became interested in individual experiences and agency as well as structural processes. Through structural approaches attention became focused on how the materiality of spaces served certain people and excluded others (Sibley, 1995). Such concerns then connected with various post-structuralist influences in the 1980s and 1990s, and geographers began exploring the ways in which exclusionary practices are contestable (Cresswell, 2009). Also at this time, social categories like age, race, gender, and sexuality were no longer assumed to be a fixed backdrop for social relations (Valentine, 2002). Space too came to be understood as playing an "active role in the construction and reproduction of social identities and, vice versa, social identities, meanings and relations are recognised as producing material and symbolic or metaphorical spaces” (Valentine, 2002, p. 146).

Relational thinking about space developed through the work of Doreen Massey who conceptualised that spaces are meeting points for the different spatial relations which produce and give meaning to them, are products of material and discursive practices, and are shaped by everyday social relations (Kitchin, 2009). Massey (1999, p. 283) said:

[Space] is the product of the intricacies and the complexities, the interlockings and the non-interlockings, of relations from the unimaginably cosmic to the intimately tiny. And precisely because it is the product of relations, relations which are active practices, material and embedded, practices which have to be carried out, space is always in process of becoming. It is always being made.

Massey stated that people are placed in different ways in relation to space. They are dependent on the relation to the flows and interconnections within it, where by "some people are more in charge of it than others; some initiate flows and movement, others do not; some are more on the receiving end of it than others; some are effectively imprisoned by it” (1994, p. 26). Social and spatial relations can therefore facilitate movement and authority, but also exclude, confine, and disempower making the relationality of space also about the relationality of power.

Elspeth Probyn's work (1992, 2003), along with Massey’s, has been enormously influential in how geographers approach space. It has also greatly influenced my aim in this thesis to "think about how space presses upon bodies differently; to realize that 
singularities of space that are produced as bodies press against space” (Probyn, 1992, p. 154; emphasis my own).

\subsubsection{What about Place?}

Tim Cresswell (2009, p. 169) sees place as “a meaningful site that combines location, locale, and sense of place”. Location refers to the 'where' or the geographic point of a place with specific coordinates; locale is the appearance or the material and tangible setting including buildings, streets, parks and; sense of place refers to the shared feelings and emotions a place evokes (Cresswell, 2009).

In any given place, says Cresswell (2009), are a combination of materiality, meaning, and practice. So, like geographers that conceived of space as material, all places have a form of materiality. Material structures like Wellington City’s Beehive building, the waterfront, or the cable-car often represent the place. On the more everyday level, towns, cities, streets, and neighbourhoods are defined by their material structures, like libraries, schools, clubs, and bars. In addition to this, all of these material things have objects and items, like people, busses, and police cars, moving through them.

Meanings can be either connected and built by an individual, or shared socially and historically. Cresswell (2009) uses the example of the World Trade Centre in New York to describe the meaning of a place. A common shared meaning of the Twin Towers was that they represented capitalism, American dominance, and phallic masculinity, which was the very reason they were attacked on September 11, 2001. It was not an attack on material structures per se, but because of the meaning of that place. On a more individual level, and linking back to humanistic understandings, we can have places that mean something to us - a place of our first kiss, family home, where a loved one is buried.

Finally, places are practised by the action of people being in these places. The imprint left by people's actions are in part what generate their meanings. Places are enacted by everyday activities like walking, going to work, driving a car. The reiteration of these activities is what gives place a sense of place.

Cresswell (2009) argues that materiality, meaning, and practice are all linked through experience. Places are prescribed with certain meanings, practices, and 'identities' and one can either experience these as inclusionary or exclusionary where it is possible to 
be 'in place' or 'out of place'. People, things, and practices labelled 'out of place' are thought to have transgressed socially-constructed boundaries that define what is appropriate and inappropriate (Cresswell, 2009), 'normal' or 'abnormal' for that place. Like my personal experience that opened the thesis, if transgression - such as that associated with the particular expression of sexuality - is pointed out through 'an inspecting gaze' it can produce self-disciplining behaviours which may limit the practice of some activities by some people. For this thesis, consideration of how sexuality as performative and as involving certain practices in relation to normative understandings of space and place is particularly pertinent.

Socially constructed boundaries of appropriate behaviour and normality vary however depending on whether a space or place is deemed to be public or private. I begin to analyse the reasons for the divide between 'public' and 'private' space.

\subsubsection{Public/Private Spaces}

Society's dichotomy of the private and public spaces are deeply rooted within spatial thought, common law, cultural/social understanding, academia, and popular discourse. The private is often associated with ideals of the family, the personal, the intimate, sexuality, and the 'natural'. While the public is seen to be the space for the state, the 'abstract', and cultural assertion (Duncan, 1996b). Duncan (1996b, p. 128) argues that this divide, which can appear to be both spatial and political, “... is frequently employed to construct, control, discipline, confine, exclude, and supress” forms of everyday life.

This divide is based on four different conceptualisations and understandings which are presented by Collins (2009) as 'axes of differentiation' to ask ourselves what we mean by private/public. These are presented as dichotomies:

1. What is political versus what is non-political;

2. What is personal versus what is societal;

3. What is hidden versus what is open, revealed, or accessible;

4. What is individual, or pertaining only to a distinct group of individuals, versus what is collective, or of concern to the whole community.

These dichotomies are important, because depending on whether a space is classified as private or public means that a different set of rules, regulations, and norms may be 
experienced by a person in them. However, Collins (2009) argues that it is possible to think of the public and the private as existing on a continuum rather than as discrete and bounded, which allows for the experience of degrees of privateness and publicness. For example, “a space in public ownership, such as a remote park or beach, might be perceived as relatively private when visited by very few people, but grow to be more public as it becomes more widely known, popular, and/or accessible” (p. 439).

What needs to be remembered here is that each space has been socially-constructed. The scenario above prioritises understandings along the third axis, but this axis may not be the most relevant when beginning to talk about aspects of the home and/or sexual activities for example.

Valentine (1993) sees our public and private lives, although distinguishable by space, as less separated than we commonly think, especially when we delve further into talking about aspects of gender and sexuality. She sees the way we represent ourselves privately is crucial to how we present our sexual subjectivities in public. Everyday, mundane interactions at work, home, school, and on the city streets do not occur between asexual beings. Rather, these interactions are between people with sexual subjectivities and these interactions mutually-construct the spaces themselves to be the product of these sexualised relationships. Sexuality, she argues, is not merely experienced behind closed doors, it is a public process. Because of this, and the ideas spoken about in this chapter already, we see that "[i]n all spheres of life we engage in this sexualized dialogue and so constantly present, negotiate and maintain a sexual identity for ourselves” (Valentine, 1993, p. 241).

Valentine’s arguments are clearly experienced in queer peoples' everyday lives, where the public/private divide is another discursive structure that limits, controls, and diminishes our interaction with space. Queer people are excluded from the construction and normalisation of private spaces, which has been symbolically conflated to be sites of heterosexual intimacy and kinship; and in public spaces, where social tolerance of queer behaviour and subjectivity can often limit our ability to express ourselves in public and resort to us being told to limit our queerness to the private space (Richardson, 1998) - but this is not always possible. 
Neat understandings of the public/private divide become even messier when considering queer private spaces like the home. The home is one of the most fundamental, but complex spaces experienced every day. It provides us with the most basic material needs but is also rich with symbolism and meanings. The home, especially in the 'West', is often constructed as a private space, but with the increase of geographic studies focusing on the queer home and domestic spaces (GormanMurray, 2007, 2012; Valentine, 1993), we see that they are also subjected to very public rules and regulations. Blunt \& Dowling (2006) (as quoted in Gorman-Murray, 2007, p. 230) note that:

Public discourse - in the media, in popular culture, in public policy - presents a dominant or ideal version of house-as-home, which typically portrays belonging and intimacy amongst members of a heterosexual nuclear family, living in a detached, owner-occupied dwelling, in a suburban location.

Home is strongly linked to the rites and rituals of the collective life of a family. This connection between home and family is a deeply-seated ideology which "valorizes some social relations and marginalizes others; defines some places as home and others as not, some identities homely and others not, and some experiences at home alienating, others fulfilling” (Blunt \& Dowling 2006, pg. 26). For queer people, limiting our sexuality or gender expression to the home is becoming increasingly difficult when something that has been seen as a distinctly private aspect of our lives - our sexuality and gender - is also further controlled in our private spaces.

These discussions on space, place, and the public/private divide are fundamental ideas that underpin the working of geographies of sexuality and queer theory today.

\subsection{Gender, Sexuality, and Queerness in Geography}

\subsubsection{Early Beginnings}

The integration of sexuality and gender within geography began in the late 1980s and 1990s when the thought of spaces and bodies being sexed and gendered and mutually constituted became conceivable (Waitt, 2010). The primary interest in the geographies of sexuality field was the analysis and mapping of social, commercial, and residential spaces of predominantly gay men and to some extent, lesbians (Gorman-Murray \& 
McKinnon, 2015). Gradually, the shift grew away from positivist views and epistemologies to more exploratory views of space, sexuality, gender.

David Bell and Gill Valentine’s Mapping Desires (1995) is often seen as the earliest and most prominent interaction between sexuality and space in geography, alongside which much of Valentine's individual work (Valentine, 1993, 1996, 2002) focused on how queer people, in particular lesbians, negotiated spaces nominally understood to be 'heterosexual' through the framing of sexuality as “... a process of power relations which mediates our everyday interactions" (Valentine, 1993, p. 246).

From then, the engagement with sexuality and gender in geography continued to grow and now includes key pieces of research that focus on spaces like the home (GormanMurray, 2007; Valentine, 1993), and the city (McDowell, 1995).

However, in the early 1990s, a critique of geographies of sexuality by queer geography saw it as too focused on identity politics. In this light, queer geography was defined and aimed to decentralise the binaries of male/female, heterosexual/homosexual, normal/abnormal, but also how these binaries structure and organise themselves in different spaces and on spaces itself (Nash, 2015). In doing this, queer geographies combines queer theory with spatial analysis to describe and understand the social construction of both sexuality and space (Gorman-Murray \& McKinnon, 2015).

A queer geographical analysis differentiates itself from other social sciences that apply queer theory by having a "particular concern with the material production and transformation of space, ... [and] relied heavily on more discursive analyses and metaphorical understandings of space” (Browne \& Nash, 2010, p. 133).

What geographies of sexuality and queer geographies has achieved together however, is “... a new visibility for the role of sexuality in how space is constituted, imagined, and experienced, and just as importantly, for the role of space in the development and constitution of sexual identities and behaviours” (Gorman-Murray \& McKinnon, 2015, p. 759).

\subsubsection{Gendered and Sexed Space}

Bell, Binnie, Cream, \& Valentine, (1994) first wrote that "space is gendered and sexed, while also gender, sex and sexuality are spaced” (p. 32). Geographers have now long believed in this idea of space, gender, and sexuality as mutually constituted. Our 
understanding now "of sexuality, sexed bodies, and sexual identities is socially crafted, and laden with the knowledge and politics that are particular to a time and place” (Johnston, 2015, p. 808).

These ideas of gendered and sexed space becomes adamantly clear in examples like the one by Probyn (1994, as quoted in Probyn, 2003, p. 80):

Consider the scene: your average type of pub somewhere ... the men are propped up on the bar, shoulder to shoulder, presenting a solid front of space gendered masculine; they are men’s men but certainly not gay. A single woman enters and she is checked over, chatted up or ignored. And if that space feels slutifying, it is because she is walking into a strata upon discursive strata that produce masculine space as the ground of differentiation and the grounds for their appropriation of women as Women.

Many other spaces are also gendered - kitchens and beauty salons are gendered female. Garages, and the example of the pub above are masculine spaces. Probyn (2003) then asks again, 'what if the woman walked in with their girlfriend?'. Not only will the woman feel their gender, they will feel their sexuality also.

The reasons we see these interactions between queer subjectivities and space is because of many intersecting power relations distributed in different spaces and places. These are understood and debated throughout all factions of queer geographies and are experienced on a multitude of different levels. They encompass the microspaces we inhabit but are also present on an overreaching and higher scale that make it hard for us to see.

Foucault demonstrated how discourses around the body have affected the control and management of individual bodies and whole populations (Valentine, 2001). Butler's theories of performativity also show how spaces, as well as bodies and subjectivities, are always becoming through their enacted performance (Browne, 2007). It has been argued that "space is made 'straight', both through heterosexual performances and also through the absence or policing of alternative expressions of sexuality” (Browne, 2007, p. 1000). Socially constructed heteronormativity also plays out in space, where norms of behaviour, sexuality, and gender control and exclude bodies and subjectivities from a space and place (seen in Probyn’s example). 
In queer people's everyday and continuous interaction with spaces that are gendered and sexed, where inclusion, access, and rights to a space can be diminished, it is important that queer people and queer youth have separate 'queer spaces' that provide them with 'safety' until binaries and discursive structures are deconstructed.

\subsubsection{Queer(ing) Space}

For decades now, queer people have been actively involved in the fight to stake claims in spatial powers. Through the queering of space, queer people are involved in destabilising the heteronormativity that is felt on a daily basis. This is important because the creation of queer spaces is fundamental for the evolution of the queer community (Rushbrook, 2002). But there is debate among queer researchers about what constitutes queer space, what is needed to create these spaces, and the ramification of these spaces once they are developed.

Sexuality and queer geography literature has emphasised the importance of queer spaces in societies where homosexual and gender-variant subjectivities are often devalued and stigmatised (Stella, 2015). Queer spaces - those created with the intention to be occupied by queer people - often involve:

... the construction of a parallel world, one filled with possibility and pleasure, while functioning simultaneously as an intervention in the world of the dominant culture, replacing its fixed principles and binary modes of thinking with the mutability of our everyday queer actions ... In its space of opportunity, we are free to construct our-selves in flexible, unspecified and unpredictable ways (Tuttleman, 2000, as quoted in Nash, 2011, p. 195)

Queer spaces occur in the margin of society (Doan, 2007). Foucault (1970) called such spaces 'heterotopias': zones of difference which hold the displaced and become sites of stability for the displaced; and Rushbrook, (2002) imagined these spaces to be bounded but permeable. They are spaces where individuals are attentive to the myriad of alternative possibilities for being, and the numerous formulations of bodies, genders, desires, and practices (Nash, 2011).

To turn heteronormative space into 'queer space' means to “implicitly recognise the heteronormative nature of most spaces and through overt action create a safe place for 
people who identity as queer" (Doan, 2007, p. 57). This is often referred to as 'queering space' and is about “... re-defining the traditionally-held norms, binaries, beliefs, values, institutions, and structures” (Freitag, 2013, p. 131).

Queer people have been queering public spaces (which have typically been seen as straight spaces) as sites of political protest in different ways to engage with, and contest public regulation and control. Bell (2009) says that since the gay rights movements prevalent in the 1960s there has been a heavy focus on claiming the right to occupy public space and resisting heteronormativity in those spaces. He explains that the most prominent resistance to public regulation of queer subjectivity in public space is the long standing tradition of pride parades and mardi gras, where pride stands against the shame of non-normative sexuality and gender. Other forms of activism like staged 'kiss-ins' and marches upon Parliaments bring attention to the inequalities present in everyday public spaces.

There are also informal queering strategies that help to undermine the heteronormative control public spaces inhibit. Some of these could be argued as the behaviours of gay men engaging in anonymous public sex ('cottaging') and seeking a sexual partner on the street or city park ('cruising') (Anderson, 2017). More subtle and overt forms of queering heterosexual spaces are the semiotics, codes, or signifiers shared between members of the queer community that counteract normalised behaviour in straight spaces. Previously, subtle codes like a certain dress sense or hair style, and the significance of a placed handkerchief, key ring, or an earring would help identify people as queer or highlight sexual preferences to other queers ${ }^{4}$ (see Figure 1), while chipping away at the control heteronormativity had on the spaces they were in.

Digital forms of queering space are seen in examples like 'Queering the Map ${ }^{5}$ ', (see Figure 2) a community generated mapping project that aims to document the spaces in which queer moments occur to produce an archive of queer experiences in space. People are able to geo-tag queer experiences which collate to produce a map of location based memories and help to reveal the way that queer people are intimately connected to each other and with space. Queer dating and hook-up apps like Grindr,

\footnotetext{
${ }^{4}$ An excellent example of these codes are documented in a book 'Gay Semiotics $\hat{\partial}$ ' and reproduced as an online index created by Hal Fischer (1977). This photo essay highlights archetypal gay images and the invention of a semiotic mode that gay men subtly use to share their sex and sexual preferences between each other. View the online essay and index at https://www.gaysemiotics.com/gay-semiotics ${ }^{5}$ View ‘Queering the Map’ at www.queeringthemap.com
} 
Scruff, and Her are also ways that queers 'remap heteronormative space' "through which [queer people] experience feelings of community and belonging, in the form of friendships, sexual liaisons or romantic relationships” and maintain connections across a digital form of space (Batiste, 2013, p. 114).

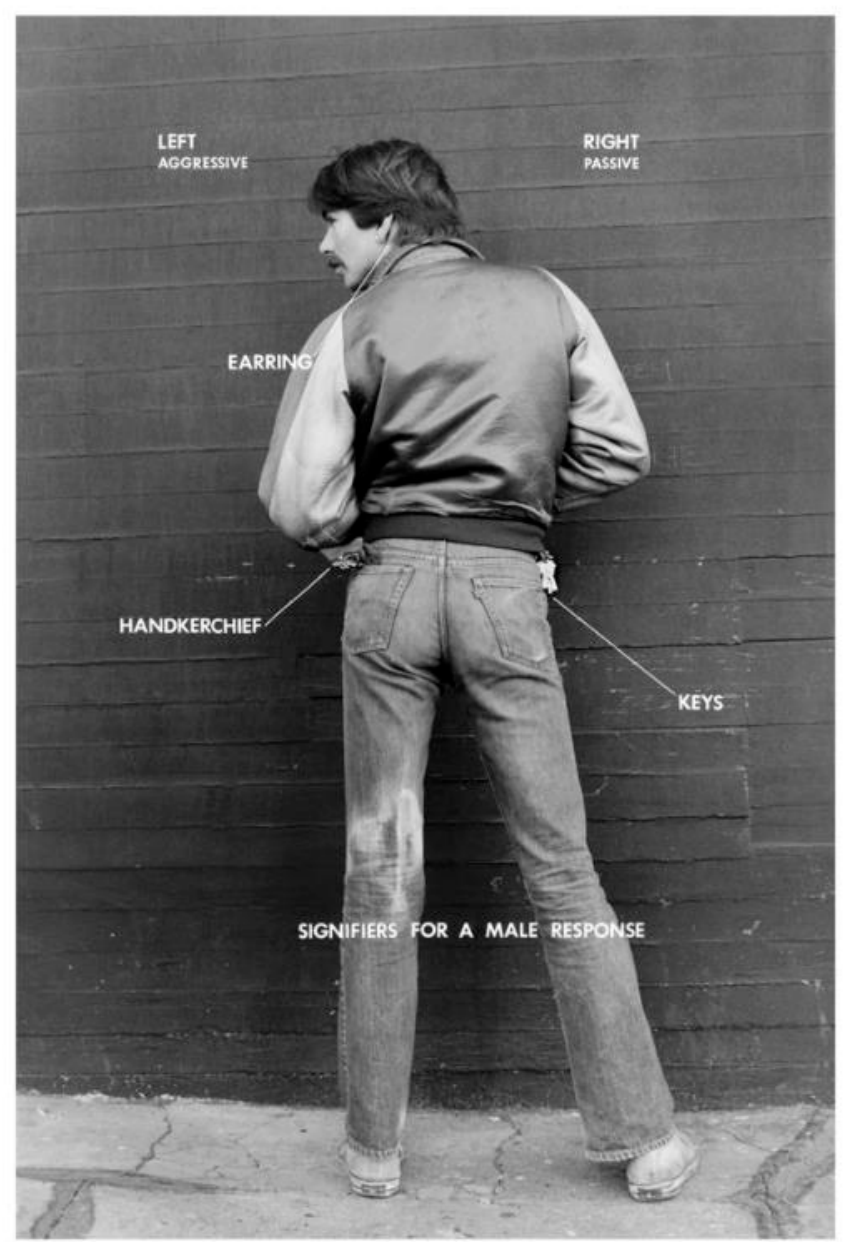

Figure 1 Signifiers for Male Response (Fischer, 1977)

To queer space is important because the inability for queer sexualities and genders to present themselves more openly within the public sphere removes opportunities for us to participate in the vitality of the public sphere and also diminishes our ability to claim our stake in wider 'spatial powers' (Duncan, 1996a). These spaces are also important as they contribute to the feeling of safety for queer people.

For queer people, the need to forge safe spaces is due to the fact that queer people are sometimes seen as dissidents within society (Rosenfeld \& Noterman, 2014), and with this status, many queer people experience significant threats to their safety. In an extensive study into the queer community in the United Kingdom, it was found that 
seventy percent of the over 100,000 queer respondents indicated that they avoided being open about their sexual orientation, while sixty-seven percent avoided being open about their gender subjectivity in fear of negative incidents (Government Equalities Office, 2018). Additionally, forty percent of respondents indicated that within the past twelve months they had experienced a negative incident with someone they did not know because they were queer, or thought to be queer. These threats resulted in some respondents indicating that they purposefully change their behaviour, clothing and appearance in public spaces so as to not be outwardly 'LGBT [Lesbian, Gay, Bisexual, Transgender]’.

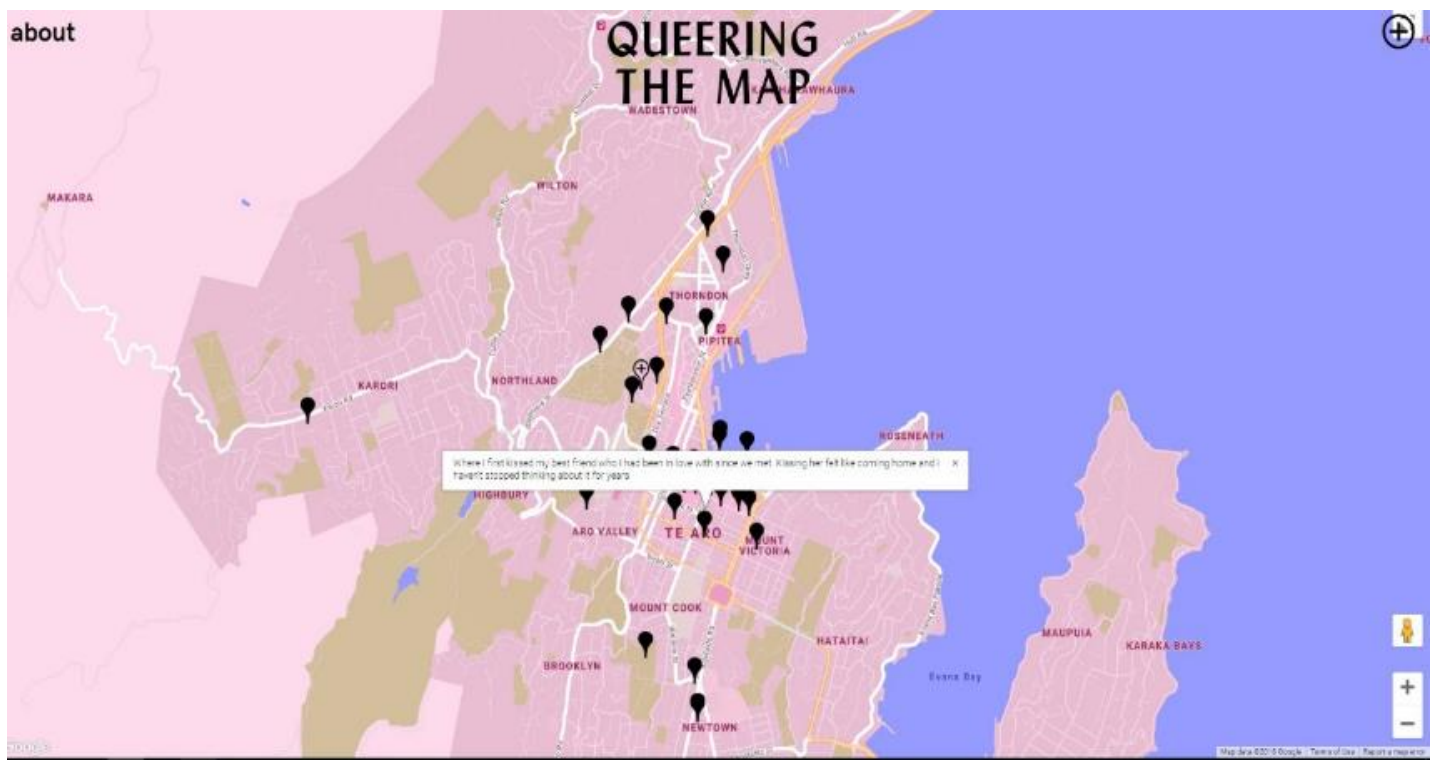

Figure 2 Queering the Map, Wellington

In contrast to these experiences, Hartal, David, \& Pascar (2014) see queer safety as a “metaphor for the ability to be honest, take risks, share opinions, or reveal one’s sexual identity” (as quoted in Hartal, 2017, p. 4). When looking at safety in the physical and spatial sense, Nash (2011) pictures a space that is free of sexism, anti-LGBT propaganda, violence, and phobia of the community. It is also a "protected place, facilitating a sense of security and recreating discourses of inclusion and diversity” (Hartal, 2017, p. 4). By reterritorialization or queering a space, it opens sites up to provide a safe space from which disempowered groups can gain control in the spatial politics of power (Duncan, 1996b). 
Hartal, (2017) outlined five major elements in the construction of safe spaces:

1. Fortification. The physical security and definitive boundaries of queer safe space makes it a site for potential asylum.

2. Anonymity. For queer people that are not publically out, or require distraction, safe spaces can be a space where no questions are asked and there is an inherent right to privacy related to the effects of fear and shame.

3. Inclusivity. The construction of safe space also needs to incorporate all queer people and where there is seen to be a right to dignity and the right to be treated as a human being.

4. Separation. In some queer spaces, the identity politics discourse of forging a collective subjectivity leads some spaces to be closed door and separated as a tool for the process of queer liberation. Examples of this include lesbian only groups and queer youth clubs.

5. Control. The need to control aspects of what happens both inside and outside these spaces is visible in most of the elements above.

There is criticism to the idea of queer space and safe space however. Debate on safe spaces often refers to the construction of queer safe space as being based on the idea that all queer people experience homophobia and heteronormativity in the same way (Fox \& Ore, 2010). Instead, it is important that the construction of safe space uses the idea of intersectionality to include other subjectivities' oppressions - like that of sexism, ageism, ableism, and racism - and their relation to sexuality. This is especially important as Fox \& Ore (2010) sees the queer safe space discourse privilege and centre around the experiences of the 'white, masculinist, middle-class' gaze (an application of Foucault's inspecting gaze within the queer community itself) which stigmatises other queer subjectivities.

Knopp (2007) also warns that although the creation of queer spaces may seem a step towards increasing visibility in space, they could be instead 'ghettoising' and bounding queers which may limit our challenge against heterosexuality, hegemony, and heteronormativity in everyday spaces. In a thought by Massumi (1992), he proclaims that “... there is an important difference between 'entrenching one's self in a closed space (hold the fort)' and 'arraying one's self in an open space (hold the street)'”' (as citied in Duncan, 1996b, p. 129). 
There are also numerous complexities as to whether creating queer safe spaces is possible. Fox \& Ore (2010) contest that there is never such a thing as a 'safe space', rather, spaces can become safe(r). The distinction between safe and safe(r) spaces is the belief that spaces can never truly be free from harm, threats, and discomfort, rather progression is measured by our ability to use different strategies to negotiate our way in an unsafe world and:

... calls attention to the tensions inherent in any discussion and action aimed to counteract multiple forms of terror and violence ... it calls to 'unfix' our definition of safety, and, instead engage safety as a process through which we establish dialogues that create and re-create spaces where queer people are more free from physical and psychic violence ... it calls us to consider the ways that safety has been too often equated with comfort around normative gender and race identities that reproduce a White male guy at the center [sic] of these spaces (Fox \& Ore, 2010, p. 643).

Fox and Ore use a metaphor of Brer Rabbit and Brer Fox to explain this, saying:

The briar patch, like the world, is a dangerous place - it is, after all, filled with brambles and that's just what keeps Brer Fox out. What makes it a refuge for Brer Rabbit is not something inherent in its nature but the fact that it's where he was raised. And having been raised there means that he knows how to move through the briar, how to use the thorns to his advantage, how to find his way through the thickets, how to, in short, negotiate its dangers successfully (Fox \& Ore, 2010).

It is possible to see these strategies employed by queer people every day. It becomes inherent in us to conform ourselves for the world to 'negotiate its dangers successfully’.

\subsubsection{Conforming and Passing Strategies}

"It is possible to be gay [only] in specific places and spaces."

(Bristow, 1989, p. 74)

Where queer bodies fall out of traditional heteronormative ideals, one becomes more sensitive, conscious, and controlled by the ways in which sexuality and gender are 
encoded and enforced in space (Nusser \& Anacker, 2013). Because of this, Nusser and Anacker say that queer people choose to perform, or edit themselves, to fit into straight spaces based on cues perceived within the spaces they move through.

Valentine's (1993) work was one of the earliest in geographies of sexuality looking into the way lesbians conceal and conform their sexual and gender subjectivities. She presented the argument that at different times, places, and scenarios, queer people must decide how, when, and if they should express their sexual subjectivity. Due to this, Valentine presented five strategies that lesbians implemented to conform and perform their queer subjectivities in different spaces and scenarios:

1. Create a geographic boundary;

2. Create different subjectivities in different spheres;

3. Limit sexual subjectivities to homes or informal gay spaces;

4. Express subjectivities at certain times;

5. Alter private spaces to conceal sexual subjectivities.

In Valentine's research, an acknowledged site of potential subjectivity conflict generated by heteronormativity was often the family home or neighbourhood, especially for lesbians who were raised heterosexual and who had not come out to family and neighbourhood friends. One of the strategies to reduce the possibility of family and friends from finding out they were queer was to create a geographic boundary between places where they were known as heterosexual and new places where they were able to create a lesbian subjectivity.

Another step further for women that were not 'out', was to create different subjectivities in different spheres so to avoid conflict between home, work, and community subjectivities. Some women described being openly lesbian at home and when partying in one city, while working in another city as straight. Another example is how the everyday conversations about private lives with colleagues, hairdressers, friends, family, doctors, and managers can be manipulated to remove any gender or pronoun identifiers for partners or by creating fictional heterosexual subjectivities.

But for women that lived, worked, and socialised all in one city, some limited their sexual subjectivity to their homes or informal gay spaces such as house parties so there was no risk of potential exposure from people they may know. Some also felt they needed to limit or conceal their subjectivity to their home because shorter hair and 
casual dresses (which is often perceived as lesbian 'style') did not fit into the norm of heteronormative spaces, like workplaces. To do this, lesbians used make-up and clothing to hide their homosexual subjectivity, project one of a heterosexual, and conform to the expectations of them (Valentine, 1993).

Valentine also drew on her previous work (1989) where she noted that public spaces can be occupied by different people at different times, so lesbians would have an idea when to express their subjectivity at certain times to avoid being outed. The final strategy she noted, is that women often altered their private spaces to conceal their sexual subjectivity, such as hiding photos and books from visiting guests.

These above strategies are categorised as being: “conscious, planned strategies” such as living away from old friends and family; "taken for granted defensive reactions" like not holding hands with a partner in public; and "everyday actions" which ingrain into our consciousness to become reoccurring and mundane strategies employed by all dissidents (Valentine, 1993, p. 246).

Carol Johnson (2002) believes that such strategies and the politics of 'passing' as heterosexual are important ways of asserting heterosexual privilege. Passing is in effect asking queer people to not do what heterosexual people $d o$, but what they $d o$ not do (not engage in same-sex intimacy and love). But in doing so can be inherently transgressive, as Sherrie Inness mentions drawing on Eve Kosofsky Sedgwick's arguments: “... the lesbian who passes as heterosexual calls into question the distinction between heterosexual and homosexual. Ultimately, she threatens to overthrow the whole homosexual order because heterosexuality can only exist in opposition to homosexuality” (Inness, 1997, as quoted in Johnson, 2002, p. 330).

Also, the constant negotiation and managing of multiple subjectivities was stressful for the lesbians interviewed by Valentine (1993). Some women felt “... 'out-of-place’ when their lesbian identity conflicted with the dominant patriarchal heterosexuality of places such as restaurants and alienated from environments such as the workplace by the need to maintain a heterosexual identity” (p. 244). 


\subsection{Geographies of Youth}

My previous engagement within queer geography and geographies of sexuality has so far focused on how space can be understood through the experience of gendered and sexed bodies. What I have yet to delve into is how age can become a factor in influencing these experiences. Here, I present the brief overview of the geographies of youth discipline and the research done within the field that contributed to the framework of this thesis.

The study of queer youth lies between two sub-disciplines of human geography geographies of sexuality and children/youth geographies - and emerged at the same as the 'cultural turn' that saw the rise of sexuality and gender integrated into geography's focus. The focus of such studies centres the youth as its subject and seeks to understand, and make transformative changes to, what could be argued as a disempowered group within society.

Like their adult counterparts, queer youth are located within the discursive structures that normalise sexuality and gender subjectivities, but they must attend to age related disparities also. In relation to how these play out spatially, queer youth's everyday experiences of space is largely dependent on the norms and regulations generated from adults and adultist practices (Schroeder, 2012). These regulations can be formal (through laws) and informal through everyday practices (curfews and rules created by parents and guardians). In both cases, geographers have studied these age based boundaries to accessing different spaces for youth and queer youth. A lot of these challenges revolve around how youth and queer youth fit into the different spaces within the public/private dichotomy.

In private spaces, like the home, queer youth are situated within the norms and regulations instigated by their parents, family, and household which sees, “... teenagers have limited ability to manipulate private property. They can’t own it, can’t modify it, can't rent it. They can only choose, occupy and use the property of others” (Childress, 2005, p. 196). Their limited control and ability to make spaces their own sees many youth occupy public spaces, such as malls, streets, and parks (Vanderbeck \& Johnson, 2000) to allow them to be situated away from the parental gaze (Rodó-deZárate, 2015). However, youth are also met with discerning views within the public space. 
Evans (2008) notes that socially constructed values of public spaces sees, “... young people's presence outside the home as problematic, and so young people [are] absent from consideration in the design and planning of public space” (p. 1659). Youth presence in public spaces is often perceived as loitering and polluting to the atmosphere of the space (Valentine, Skelton, \& Chambers, 2005).

For this reason, and the reasons also felt by queer adults, there is a real need for queer youth to have access to safe spaces. Such spaces can offer individuals the opportunity to escape away from forms of discrimination, violence, and heteronormative discourse to provide the ability for positive self-determination and character building away from an adultist gaze (Valentine and Skelton, 2003).

Quite often it is perceived that the bedroom provides such opportunities for youth. However, their construction ultimately effects the level of privacy and ownership a young person has over the space, and as such, a bedroom “... becomes a locus for privacy and disclosure, dating and romance, peer pressure, and parents' or guardians' authority” (Schroeder, 2015, p. 788). For queer youth, the bedroom, can be a place of acceptance and protection but also a danger. Schroeder (2015), drawing on Michael Brown's (2000) Epistemology of the Closet, says that:

Like the closet, the home and even the spaces within it such as the bedroom, can be a place that is accepting, even protective from the outside world, but it can also be a place that is unstable - even dangerous. For queer youth, the bedroom becomes an important space they can choose for their own privacy, or are banished to, due to other circumstances. And, for queer youth, the bedroom offers some similarities to the closet as both the bedroom and closet can conceal protectively or trap oppressively. It can be a prison or a sanctuary - and often both simultaneously (p. 795).

The school is also an everyday space where queer youth engage with heteronormative enactments both in their relationships with other students and teachers, but also expressed in the institutional practices and policies (Toomey, McGuire, \& Russell, 2012).

Reflecting on the work already conducted with youth and queer youth in geographies of sexuality and children/youth geographies, this research aims to contribute not only to these sub-disciplines to further build an understanding of the everyday spaces 
discussed here, but also space and places that have been neglected in geographic research so far. It also aims to uncover where queer youth feel safe and the elements of spaces that contribute to that feeling.

\subsection{Chapter Summary}

I began chapter three by presenting the diverse ways in which geographers have conceptualised space and place and how the terms will be used in my research. By detailing the development of queer theory, sexuality, and gender within geography, I outlined where my research currently sits within the sub-discipline of geographies of sexualities. Previous research on gendered and sexed spaces, and queer(ing) space, has provided an understanding of how other geographers have viewed queer experiences of space and places. It guides my approach to studying the gap that was identified the lack of queer youth in geographic research and even fewer from a collaborative and transformative approach. 


\section{CHAPTER FOUR: METHODOLOGY}

In this chapter I discuss the epistemological and methodological approach I took to conducting research on the everyday experiences of a purposeful sample of queer youth in Wellington city. I begin by presenting my epistemology and justify its relevance for this research topic. I then outline the photovoice methodology including participant recruitment, the role of photography, the use of focus groups, and the production of a zine. I also detail why more-than-visual, sensory, and bodily experiences are important in this study. Next I discuss my use of Foucauldian discourse analysis through which I analysed the photographs and narratives of participants. Finally, I discuss ethical considerations, and my positionality, in this research.

\subsection{Epistemological Approach}

This research is guided by a transformative paradigm and in doing so has placed central importance on the lived experiences of communities on society's margins, analyses power relationships, and links results of the research to action (Mertens, 2008). This approach is important because it recognises the explicit political dimensions of exploring sexuality and space and is driven by "a political change agenda to confront social oppression at whatever level it occurs” (Creswell, 2014, p. 9). It places central importance on understanding and recognising the lives and experiences of queer youth who have traditionally been marginalised and constrained by oppressive strategies. It also enables me to work alongside participants to advance an agenda for collaborative and collective change (Creswell, 2014).

\subsection{Queer, Multi-Sensory, and 'Everyday’ Methodology}

Within a transformative approach, the research adopts a queer qualitative methodology which aims to highlight the lived experiences that fall outside of current normative binaries; to understand possible other ways of being; and to make visible the discriminatory and exclusionary regimes of heteronormativity (Nash, 2015).

A queer qualitative methodology embraces sex and gendered subjects as fluid, blurred, and contingent on discourse, power, and control, while working with this understanding in embodied and experimental ways (Browne \& Nash, 2010). For many 
researchers, these have involved a focus on interviewing and story-telling privileging verbal or written narratives. However, increasingly visual methodologies have been recognised for their ability to enhance research enquiry (Reavey \& Johnson, 2017). Firstly, visual methodologies allows researchers to gain access to information that may have been hard to reach through other routes:

Written descriptions, and interview and survey methods, can go some way towards both recording and presenting such research, but film and photography not only enrich such studies, they can provide insight beyond that which is possible with mere words alone (Banks, 2007, p. 31).

Secondly, Frith \& Harcourt (2005, p. 189) believe that such methodologies, when engaged in a research project produced with PAR principles, can "change the voice of the research" by allowing participants to be heard and present opportunities for personal and social transformation. Thirdly, the presentation of research findings in a visual format can permit a wider engagement with outside audiences.

In addition to privileging the visual, geographers are beginning to participate in multisensory and more-than-visual methodologies to better understand how bodies are placed within spaces. To include other sensory experiences of space acknowledges that “... our phenomenological experience is not of disjointed sensory sensations but is instead of a coherent multisensory world, where sounds, smells, tastes, lights, and touches amalgamate' and our sensory modalities 'combine, substitute, or integrate” (Newell \& Shams, 2007, p. 1415).

In this light, it is important for this research to incorporate and reflect on Sarah Pink's (2011, p. 603) research principles to help guide the research project to embrace the multi-sensory elements in visual imagery projects:

1. Visual images are produced and consumed in multisensory environments; they stand for the multisensory configurations from which they emerge;

2. Visual images are not simply visual; they are experienced through multiple and intertwined sensory channels;

3. Vision itself is a culturally constructed category, as are sound, smell, taste, and touch;

4. Vision involves more than just looking at images, and visual practices need to be situated as part of multisensory perception. 
In addition to privileging the multi-sensory experiences of space and their portrayal in visual imagery, it is critical for this research to value and recognise the everyday, mundane interactions with space and place that occur under different discursive regimes.

The study of the everyday is significant within many different disciplines. It is a critique of approaches that favour the macro-level theory and understandings of space where a person is seen to be often a passive surface that internalises norms and values (Courpasson, 2017). Courpasson, (2017, p. 844) says that:

The study of everyday life started from the premise that understanding people's real lives involves analysing their experiences in their 'natural' context, that is to say, where they accomplish things and deal with the hard matter(s) of actual existence: the everyday social world explains how societies are constituted.

When studying the micro, everyday things and doings one can reveal the larger macro structures that obscure the spatial and political texture of everyday life (Courpasson, 2017) - and this is important. Courpasson (2017) believe that people are not passive subjects to discourses, rather we have the possibility to subvert and amend them (think queering space) but only at the everyday level. This is because despite the constraining forces in space, there are interplays of other forces that can help us overturn these through the reconceptualising of the everyday ordinary to the extraordinary.

Lefebvre (1991) uses the example of the café and highlights what makes it extraordinary and riddled with subjectivity, power, and discourse. A café is:

Generally an extra-familial and extra-professional meeting place, where people come together on the basis of personal affinities (in principle and at least apparently), because they have the same street or the same neighbourhood in common rather than the same profession or class (although there do exist cafes where the clients are predominantly of the same class or profession). It is a place where the regulars can find a certain luxury, if only on the surface; where they can speak freely (about politics, women, etc.), and where if what is said may be superficial, the freedom to say it is fiercely defended; where they play (p. 63). 
The view of the everyday has been linked to repetition and veiled by obsession and fear (Courpasson, 2017). But Lefebvre argues that we need not study the everyday as if it were mundane because mundaneness is not always meaningless. He poses the question “why wouldn't the concept of everydayness reveal the extraordinary in the ordinary?' (p. 9).

These approaches I have detailed were implemented and applied in this research to an already visual method, photovoice, to account for the relationship between multisensory lived experiences of queer youth in everyday space.

\subsection{Photovoice as a Research Method}

\subsubsection{Introduction to Photovoice}

Photovoice was developed in the 1990s (then called photo novella) by Wang and Burris as a research method "by which people can identify, represent, and enhance their community through a specific photographic technique” (Wang \& Burris, 1997, p. 369) and was first used in a 1992 project in rural China which looked into improving women's health and to empower women to mobilize social change. Since then, photovoice has grown to become a popular PAR tool that focuses on working with not just women, but also with other marginalised peoples and communities (Nykiforuk, Vallianatos, \& Nieuwendyk, 2011).

Photovoice's aim, informed by its Freirian underpinnings, is to enable participants to use photographic images to represent and reflect their community's strengths and concerns from their own point of view; promote a critical dialogue and understanding about community issues through group discussions and sharing of photographs; and reach policy makers and society at large by communicating issues with both concern and pride (Wang \& Burris, 1994, 1997).

It also allows participants to be active in understanding these realities, and through the sharing of mutual experiences, become agents for social change (Strack, Magill, \& McDonagh, 2004), and make participants aware of their own responsibility to maintain or make changes in their reality (Carlson, Engebretson, \& Chamberlain, 2006). The intended outcomes for participants is threefold: To become empowered, provide the ability to assess community needs, and to take action in the community (Strack et al., 2004). 
In previous photovoice projects where youth or queer youth have been involved, the feedback from the participants suggested that the whole process was in some way empowering (Strack et al., 2004), and a novel mode of self-expression (Holtby, Klein, Cook, \& Travers, 2015; Strack et al., 2004). Youniss \& Yates (1997) believe that photovoice provides the opportunity for youth to build and confirm their own abilities, to provide input, share experiences to the wider community, and to develop a 'social morality' while becoming positive agents for social change.

To introduce and embrace multi-sensory dimensions of experience into the photovoice project, participants were asked to take photographs when they felt, experienced, or had a particular connection to a space or place. In doing so, it brought out more detailed, reflexive, and embodied responses from participants. In getting participants to reflect on the photograph and the experience felt at the time allowed participants to develop connections with time, imagination, sound, touch, and memories that may have been difficult to create with purely directing participants to focus on the visual aspects of their experiences with space.

The implementation of a photovoice method in qualitative research varies across different studies and is dependent on time, budget, participant numbers, and the overall end goal of the research. In the case of this research, the time needed to complete the photovoice project from start to finish took three and a half months and also required the participants to meet as a group three times over three separate weeks, take photographs for three weeks, and meet for a one-on-one interview for two hours. However, regardless of these, Wang (2006) outlines a nine-step strategy that a photovoice project typically involves:

1. Select and recruit a target audience of policy makers or community leaders.

2. Recruit a group of photovoice participants.

3. Introduce the photovoice methodology to participants, and facilitate a group discussion about cameras, power, and ethics.

4. Obtain informed consent.

5. Pose initial theme/s for taking pictures.

6. Distribute cameras to participants and review how to use the camera.

7. Provide time for participants to take pictures.

8. Meet to discuss photographs and identify themes. 
9. Plan with participants a format to share photographs and stories with policy makers or community leaders.

I adopted this process and discuss each element below.

\subsubsection{Target Audience and Partner Organisation Recruitment}

To change perceptions and norms relating to aspects of gender and sexuality in spaces, it is important that this research reaches the local community to raise awareness of queer youths' experiences of space. It is hoped that with this new awareness, more people become allies and supporters of queer subjectivity and de-stabilise heteronormativity and discursive structures.

At an institutional level, it was also important to present the research to leaders and organisations in the everyday spaces commonly visited by the queer youth, such as schools and local councils, where institutional changes that promote discursive strictures could be highlighted.

To reach these target audience groups, I partnered with the InsideOUT organisation. InsideOUT helps to set up and provide support to school groups (called queer straight alliances or QSA) in Wellington. Here, queer students can meet on a weekly basis to socialise, discuss issues, organise projects within their school, and receive support on topics such as positive mental health and queer sexual education. It is also has a strong presence within the local community and provides support to local council, central government, and businesses on the issues facing the queer community.

In this partnership, I worked independently with my own research questions and aims but was helped by the organisation to recruit participants, direct my line of enquiry in the focus groups, and disseminate the final result of the project. In providing the endresults of this research to InsideOUT, it is hoped that this would increase potential impact and the likelihood of reaching the key stakeholders identified to facilitate positive change.

Although InsideOUT provided input into my research questions, I approached them for support with an already set research agenda. Where more time is permitting, I would have liked to have brought the leaders into the earlier stages of the research and got them to help with the construction of the research questions. 


\subsubsection{Participant recruitment}

Participants were recruited with the help of InsideOUT's Wellington QSA network. I was welcomed into two of these meetings in June 2018 at two local high schools - one co-ed secondary school, and one single-sex 'female' intermediate and secondary school - to explain my research and ask for potential interest by the students.

From the queer school groups that I attended, only two participants signed up to the project because a lot of the students who attended these meetings were under the age of 16 and so were outside of my target group ${ }^{6}$. Because of this, I decided to expand my recruitment to also focus on queer youth outside of QSA meetings and include the experiences of 'older' youth (19 to 24 years) too. I posted on the InsideOUT school leaders' Facebook group which helped reach out to other high school students and older queer youth that had once been a part of, or volunteered for, a school group. The Facebook post proved to be the best way to recruit participants with five people signing up to the project over a two week period. At the conclusion of the recruitment process, seven people were interested in taking part in the photovoice project. Half way through the project, one participant withdrew from the study due to personal reasons, leaving six queer youth.

\subsubsection{Introducing Participants}

All participants were someway involved with the InsideOUT organisation and identified as queer. All participants had access to a camera, or a phone that had a camera built-in. They were aged between 16 to 22 years and had different cultural, religious, and family backgrounds; diverse involvement in queer activism; and all were out to friends and family in one way or another. All but one participant were gender non-binary or preferred genderless pronouns. ${ }^{7}$ Two participants identified with a sexual subjectivity of pansexual while the others either preferred not to say, or did not identify with another sexual subjectivity apart from queer. All participants wanted their real names used throughout the research process. I introduce you to them here:

\footnotetext{
${ }^{6}$ Due to concerns raised by the Victoria University ethics committee and InsideOUT, I limited my recruit participants over the age of 16 years old. The reasoning and implications of this will be discussed further in section 4.4.1.

${ }^{7}$ Participants that preferred the use of gender-less pronouns are referred to as they/them, instead of he/him or she/her in this thesis.
} 


\section{Compass, 17, They/Them, Queer}

Compass is a student leader at their high school's QSA where they have started to attend after previously being home-schooled. Compass is actively involved with InsideOUT and enjoys photography and nature. Compass, whose family is originally from Germany, has lived here most of their life.

\section{Lillian, 16, She/Her, Female, Queer}

Lillian is an active leader in her high school's QSA group and enjoys talking about the queer community at the school, organising events, meetings, and presentations for other members of her school. She has recently finished organising the school's pride week which was seen as being a great success.

\section{Shay, 20, They/Them, Queer}

Shay is from a Māori whānau and goes to university. They are extremely passionate about art, graphic design, and performing arts and are motivated to take up opportunities and experiences to learn new things. Shay is a hardworking and driven student and relaxes by playing with animals and practising their art.

\section{Charlie, 22, They/Them, Non-binary, Pan Sexual, Queer}

Charlie is a recent university graduate and lives out of the city centre with their partner, Nich. Charlie enjoys spending time with their partner, walking their dog Robbie, and being near the ocean. From a Chinese family, Charlie speaks their native language and enjoys sharing their culture with Nich.

\section{Nich, 22, They/Them, Pan Sexual, Queer}

Nich also enjoys similar things to their partner Charlie. Still at university, Nich likes the small moments in life and spending time at home with Charlie. Also being Asian, Nich is finding living with Charlie an opportunity to learn more about their culture and to develop their understanding of Chinese language.

\section{Kate, 20, They/Them, Non-binary, Queer}

Kate is an active member of the InsideOUT team, working as a volunteer and devoting plenty of their time to improving the lives of queer youth in Wellington. Always on 
the go, Kate likes performing arts, art, and poetry and has a queer positive blog and Instagram pages.

After recruitment, participants began the project by attending the first of two workshops.

\subsubsection{Pre-photo workshops}

Following the photovoice strategy by Wang (2006) and reviewing previous experiences in working with youth and photovoice presented in other studies, I decided to organise two pre-photo taking workshops with the participants. The aim of these workshops were to generate relationships between participants and to work through the necessary information participants needed to undertake the photovoice process.

The workshops were held at Toi Pōneke Arts Centre in Wellington city. The space was large and inviting which facilitated us being able to sit on the floor in a circle and share kai (food) and a kōrero (discussion) about the project, ourselves, and our experiences (Figure 3). The space was chosen due to its purpose of fostering art within the community, its proximity to participants, and its ease of access.

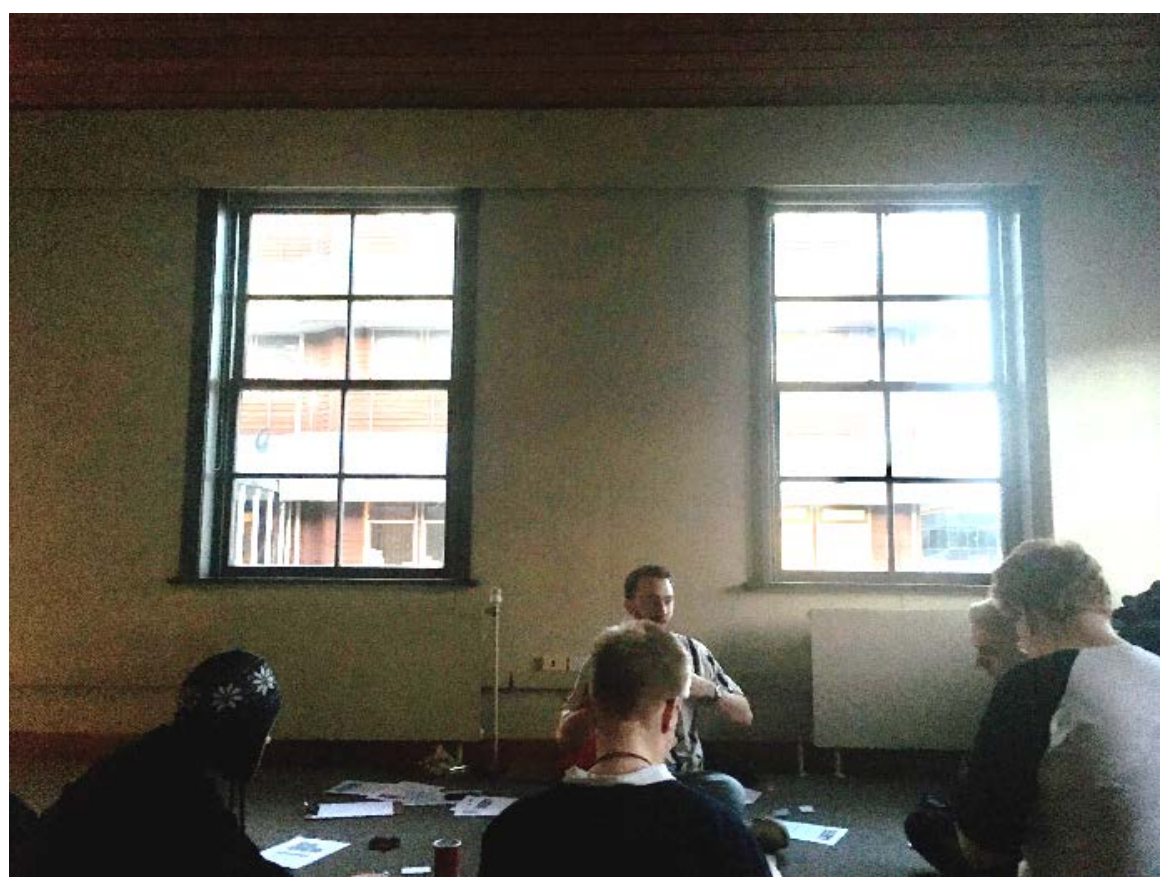

Figure 3 Focus Group Two at Toi Poneke Arts Centre (Lillian) 
The first workshop began with an energizer game and with us all creating badges with preferred names, ages, and gender pronouns. We then completed two activities aimed to get the participants thinking about feelings, experiences, and emotions in different spaces and linking those to ideas of aspects of straight and cisgender privilege. To do this, participants were asked to draw out a timeline of their day so far and to link an emotion or feeling to each space they had been in or passed through. Although these feelings did not have to be linked to their queer subjectivity, the activity helped participants begin to think about how they feel in different everyday spaces. Two examples are shown in Figure 4.
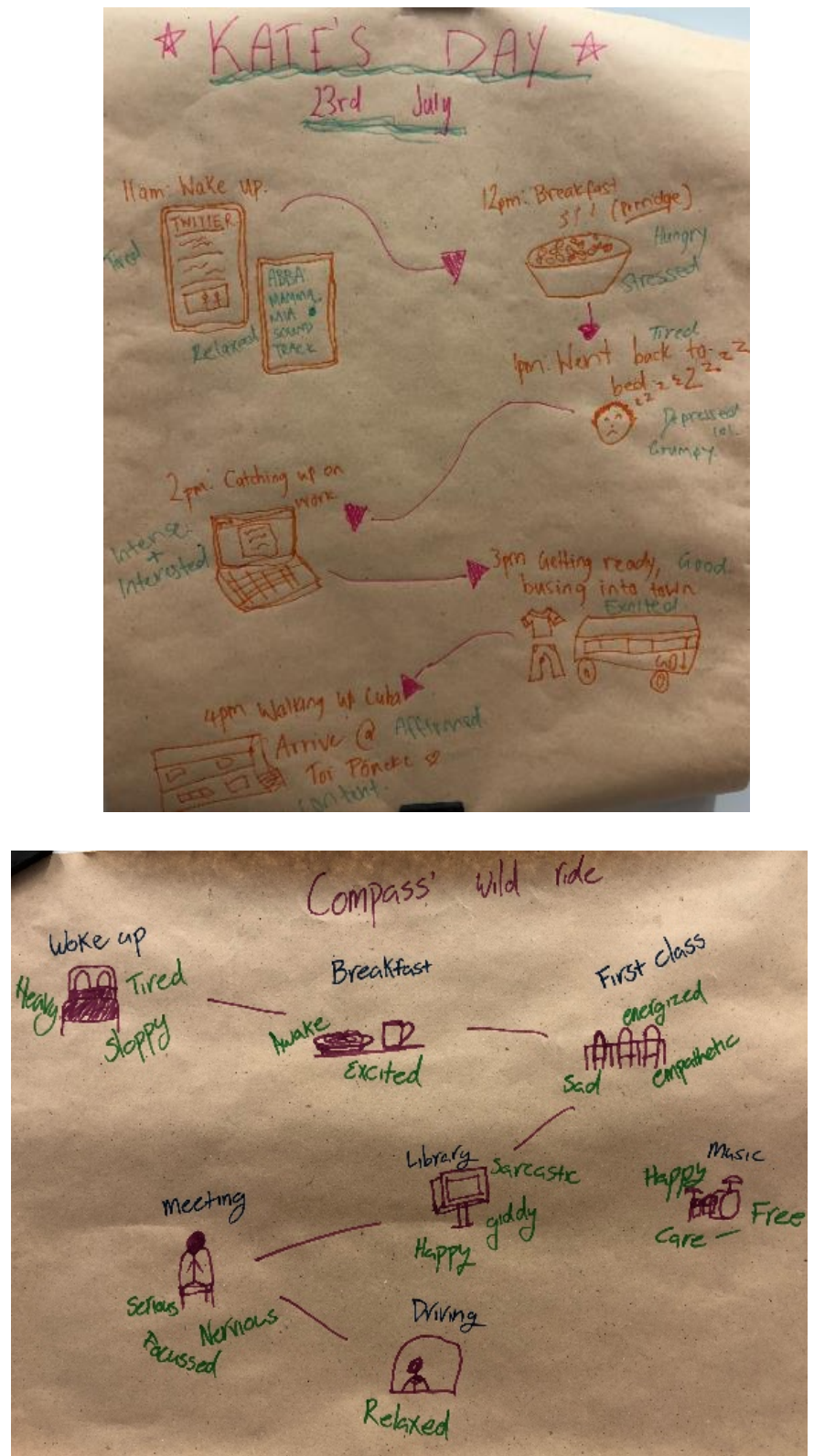

Figure 4 Compass and Kate's Day Focus Group Activity 
The next activity was to get participants to start thinking about aspects of straight and cis-gendered privilege. Participants filled out a worksheet independently which asked them to say whether or not they experience aspects of heterosexual and cisgender privilege. The worksheet (see Appendix A) was based on a similar activity titled 'Unpacking the Invisible Knapsack II' adapted from a resource by Peggy McIntosh (1988). It aimed to generate conversation and thoughts by the participants on how their day-to-day activities, interactions with spaces, and privilege were often different to that of a cis-gendered heterosexual (cis-het). Most of the participants responded that they in fact do not experience the same privilege as a cis-het.

Participants left the first workshop with an information sheet (see Appendix B) which gave guiding ideas on what aspects to think about when they were in the photo-taking period and I welcomed questions at the following workshop a week later once they had time to read over the information.

The second workshop involved further relationship building with participants and delved into brainstorming different techniques and approaches for taking appealing, meaningful, and unique photos. These ideas were further supplemented and adapted from a photovoice resource of different composition techniques to convey meanings in photographs (see Rutgers, 2016). These included discussions on angles, lighting, and the 'line-of-thirds' technique. This first activity was well received and most participants were all very knowledgeable on photographic techniques from previous art and photography backgrounds. A talk on health and safety followed that involved reading over an information sheet (see Appendix C) which was comprised of general reminders about aspects of personal safety, ethical issues associated with photographing other people, and the power of representation. The workshop concluded with participants being able to ask questions about the project as a whole.

\subsubsection{Taking the Photographs}

After the workshops, participants were given just over three weeks to take their photographs of different spaces. The time frame was chosen to allow plenty of time for participants to take different photographs of varying spaces, including ones visited over the weekends. They were tasked to take photographs of spaces that portrayed their everyday experiences as a queer youth. I told the participants to 'think queer', 
use their emotions as a cue, to think about their safe and unsafe spaces, and to have fun.

Although participants were able to take as many photographs as they chose to, previous photovoice research recommended to limit the number of photographs participants were to send back to the researcher. In this instance, I provided a limit of 25 photographs which forced participants to think about the meaning behind photographs and choose the ones that they thought best represented what they wanted to portray. It also allowed myself and the participants to better group photographs into themes in the interview stages.

\subsubsection{Follow-up Interviews}

Once the participants had concluded taking photographs, I scheduled an hour and a half semi-structured interview time with each participant at the Wellington Central Library in a private meeting room. Each interview was audio recorded with a voice recorder with the participant’s permission.

Semi-structured interviewing employs the use of an interview guide (see Appendix D) where talking points, themes, and formulated questions were constructed but were not deployed rigidly in the discussion (Dunn, 2005). Questions and themes of discussion were shifted by the flow of the conversation. New themes and questions developed as participants were asked to answer the following questions for each of their photographs:

1. What does this picture show?

2. Why did you take this picture?

3. What were you thinking/feeling at the time you took this picture?

The questions followed a similar, but not the exact, format used in Wang and Burris' SHOWeD model (1994). Instead, I allowed the participant to explain, in their own words, the reasons for taking the photographs. I used probing questions to encourage participants to further think and describe certain themes. It was important that in these interviews that the participants had the opportunity to explain and share with me what the photograph represented. It also allowed me to uncover what they were feeling and experiencing at the time of the photograph - something the photograph and my interpretation alone would not have told me. 
After questions were finished, participants then grouped their photographs into the themes they were wanting to portray. These were noted down and then discussed. Lastly, participants were required to choose two or three photographs from each theme to take forward into the focus group. Only two participants had to complete this stage as the other youth had taken only a few photographs per theme. Captions were then added to these photographs by the participants in their own time after the interview.

\subsubsection{Focus Group}

After the individual one-on-one interviews were conducted, everyone met back at Toi Poneke Arts Centre for a ninety minute focus group session. Audio recordings and notes taken by a volunteer note-taker were captured for future analysis.

The focus group was an important tool to use within a research setting that aimed to understand the multiplicity of meanings and experiences of similar people and supplemented the individual interviews done with participants. It provided the opportunity for people to explore each other's view while formatting and reconsidering their own ideas. It also produced shared and common understandings and experiences between participants that would not have been able to be collected solely from the interview process, while also allowing participants to vehemently disagree with each other (Cameron, 2005).

\subsubsection{Transcription and Member Checking}

After the interviews and focus groups had concluded, I transcribed the audio recordings verbatim. I decided against using transcription software or services so I could get re-familiar with the interview and focus group discussions while also giving me the opportunity to add in notes about witnessed social cues that were unable to be picked up by the reorder (Dunn, 2005).

Although not part of Wang and Burris' photovoice steps, participants were also given the opportunity to review, edit, and read over their transcriptions for 'participant checking'.

\subsubsection{Analysis and Interpretation}

Linking in with my epistemology and theoretical framework, my approach to analysing the transcripts and interpreting the photographs aimed to accurately portray 
the experiences of the queer youth, and explore how these experiences were felt through the working of certain discourses (Potter \& Wetherell, 1994).

As explained in chapter two, Michel Foucault was interested in the way discourse shaped understandings and expressions of subjectivity in the body. His approach to discourse analysis sought to "uncover the social mechanisms that maintain structures and rules of validity over statements about particular people, animals, plants, things, events and places” (Waitt, 2010, p. 218). Thus the knowledge presented by participants was not uncritically understood as the Truth, but rather as a product of the mutual constitution of their subjectivities and particular spaces within discourse.

Before beginning discourse analysis, it was imperative that I acknowledged my preconceived notions on the topic and placed them aside to find the structures that account for the 'naturalness' of categories and subjects. Foucault (1972, p. 25) wrote that preconceptions:

... must be held in suspense. They must not be rejected definitively, of course, but the tranquillity with which they are accepted must be disturbed; we must show that they do not come about by themselves, but are always the result of a construction the rules of which must be known and the justifications of which must be scrutinized.

It was also important to acknowledge my positionality (see Chapter 4.4.2) and to practice reflexivity throughout the research process and analysis.

I began the analysis by organising the interview and focus group transcripts with descriptive codes. These descriptive codes were generated paying particular attention to Waitt (2010)'s four category labels:

1. Context (when, where, who).

2. Practices (the events, interconnections, and actions).

3. Attitudes (statements).

4. Experiences (feelings about attitudes).

These labels helped to initially group quotes and themes into codes, which were ever changing and growing as the process went on. 
I then explored these quotes, codes, and themes to understand how knowledge was being sustained as truth through particular discursive structures. In Western rational thought, these structures often take the form of dichotomies - public/private, man/woman, gay/straight, safe/unsafe, and masculine/feminine, which tend to fix ideas about how the world is or should be experienced by particular groups of people. Hence, when doing discourse analysis it was important that I was aware of the discursive structures that lead some knowledge to become understood as "valid, legitimate, trustworthy, and authoritative” (Waitt, 2010, p. 233) by my participants, and how other knowledge was marginalised or silenced. Rose (2001) noted that the invisibilities and silences in data can be just as powerful as what is visible or spoken. Foucault highlights how privileged and dominant discourses operate to silence different understandings of the world and it was important to consider how different subjectivities and spaces were positioned, and which were accorded more authority within participants’ interviews and discussions.

Discourse analysis also explores inconsistencies within different sources. While discursive structures may appear fixed and natural, they are also fragile and open for contestation and reconstruction. Therefore I also paid particular attention for possible contradictions and opportunities for resistance or reconstruction in my participants' narratives.

Discourse analysis can also be used on drawings and imagery. Within geography, Rose (1996, p. 280) says that "visual representations of places, spaces and landscapes are never taken as straightforward mirrors of reality, ... instead, the meanings of an image are understood as constructed through a range of complex and thoroughly social processes and sites of signification”. She insists that when analysing visual imagery, it is best to use the image as a function that produces a particular representation of the world, to inquire into its significance, and elucidate the ways in which social powers have structured the meaning portrayed in the image (Rose, 1996). This required a balance between analysing participants' photographs independently, with reference to the participants' experiences, and in combination with other participants’ photographs.

To attend to the multi-sensory and more-than-visual analysis of the queer youths' experience represented in the photographs, I followed Pink's (2011) principles of enacting more-than-visual research discussed earlier and viewed photographs as: 
... the outcomes of multisensory contexts, encounters, and engagements. The act of taking a photograph involves the convergence of a range of different social, material, discursive, and moral elements in a multisensory environment, rather than being a solely visual process (Pink, 2011, p. 602).

These photographs were analysed alongside the participants' interviews and focus group discussions to understand this convergence of various elements at the very time they took the photograph. In doing so I was able to elicit how discursive structures and their effect on queer youth were experienced on the body (through sight, sound, smell, touch etc.). Participants were also given the opportunity to edit their photographs to portray these multi-sensory experiences to the audience in a visual format.

\subsubsection{Dissemination and Reflection}

The final step of a photovoice project which contributed to the transformative and participatory aspects of this research, is to disseminate the knowledge and experiences discovered in the project.

After the conclusion of the photovoice project, the participants decided they would like to present their photographs and experiences in a zine format. Zines are usually presented in a small booklet format with images, texts, and drawings. Zines are seen as "grassroots, underground movement and have increasingly become a vehicle for the voices, ideas and feelings of more vulnerable populations and those who experience discrimination from the dominant culture” (Desyllas \& Sinclair, 2014, p. 299). Used as a tool to bring to light social injustices in an artistic and creative medium, zines also enable critical thinking and opportunities to challenge the mainstream culture on the part of the maker and on the readers (Desyllas \& Sinclair, 2014).

A zine is currently in the process of being created with input and design ideas shared collaboratively. Once completed, this will be distributed to InsideOUT for use in their campaign towards improving queer youths' everyday lives. It is also hoped that this will be distributed to local and national libraries to be made available in their zine collections. Participants will also have access to copies for distribution to friends, family, and their schools. 
In producing this zine and making it available to the wider Wellington community, it is hoped that this will generate interest and willingness by people to change the normative values in spaces where heteronormativity is profoundly visible.

After the completion of the photovoice project, I asked participants to reflect on the photovoice process and the feelings and experiences they had been through. All participants agreed that the process had made them think of their everyday space and subjectivity differently and were interested in producing the zine to share these new understandings of spaces outside of the group.

\subsection{Ethics, Positionality, and Representation}

It is important that I acknowledge my role and positionality in the research and be reflective on how these could have influenced the results of the project. Additionally, it was also imperative that I draw on transformative and participatory orientations in my methodology to bring forward change and increased awareness to queer youths' experiences of everyday space in my role as the researcher.

\subsubsection{Ethics}

An application for my research was submitted to the Victoria University of Wellington Human Ethics Committee and approved before the research was conducted. Participants were given an information sheet (see Appendix E) and consent form (Appendix F) to read over and sign before beginning the research process. An information session during one of the pre-photo workshops provided information of what was required of each participant, the ethical considerations of taking photographs of spaces in public, the ramifications this research could have on the queer and wider community, and health and safety considerations.

Participants gave consent for their photographs, transcripts, and preferred names, sexual, and gender subjectivities to be used in this thesis. The queer youth have full ownership of the photographs in this thesis and are acknowledged when their photographs are used.

From researching this topic, I found it is not uncommon for institutional ethical committees to see researching with youth as being a 'research risk'. Some researchers and ethics boards believe that queer youth “... are vulnerable, risky subjects who are disadvantaged by their social position and in need of protection within research 
processes” (Taylor \& Dwyer, 2015, p. 244). Thinking from a queer perspective, this repetitive way of thinking about queer youth soon makes this narrative appear to be normative and Truthful. The fear of working with queer minors is to further deny and disadvantage the 'at risk' young queer voices. This argument was included in the ethics application as a closing argument to my application for this research.

However, these narratives still remained visible in my research and ethics application. Youth aged between 12 and 16 years of age were not recruited for this research since the ethics committee consider them to be minors and would require a parent or guardian's signature to participate. This would become an issue during recruitment because if minors did want to take part in the project, they may be required to 'come out' to participate, potentially putting them in a position of distress. I have made some reasonable attempts to combat the inability to work alongside under 16 year olds by asking questions during the research process about participants' previous experiences as ‘younger' youth.

\subsubsection{Positionality}

As an out gay and queer man, I was positioned in one sense as in 'insider' to my research and my participants. This provided me with knowledge and first-hand experience into what the queer youth were discussing and enabled me to understand and relate with some common experiences of the participants. However, I was also positioned as an 'outsider' to some of the participants as I am a cis-gendered man, from a middle-class, well-educated, Pākehā New Zealander background. In instances such as discussion on gender non-conforming and Māori experiences, it was important that I listened, learned, and was reflective of other people's positioning with the queer subjectivity that I was unfamiliar with.

My positionality and role as researcher also meant that I needed to be careful about my assumptions when working with participants’ images and narratives to ensure my interpretations were as trustworthy and robust as possible (Dowling, 2005).

\subsubsection{Representation}

Before interpreting the results, participants had the opportunity to member check their interview and focus group transcripts to allow participants to redact, alter, or reaffirm their position on a question or topic. Each participant had the opportunity to provide a caption to their photographs that would be displayed in this thesis and in future 
publications. In doing so, it allowed the participants voice, experience, and understanding to be portrayed next to their photograph and guide my interpretation and summary of their experiences.

My own interpretations and analysis of the participants' experiences and photographs is also present within the discussion section of this thesis. These interpretations do not aim to homogenise or make generalisations of the participants' ideas and experiences, rather they focus on presenting embodied and everyday experience of spaces and attend to the higher level discursive structures and geographic understandings of space that created these experiences.

\subsection{Chapter Summary}

In chapter four, I detailed my methodological approach to this research and provided a step-by-step account of the processes involved in collecting, analysing, and disseminating this qualitative, transformative research with the queer youth participants. I provide justification for the need to focus on the everyday and multisensory experience of space and subjectivity to attend to the larger discursive structures and create embodied research data and results. The processes of photovoice and Foucauldian discourse analysis were outlined to show how participants' photographs and experiences were generated and analysed. Lastly, I reflected on ethical considerations, my positionality, and ideas around representation to be upfront about how these could have influenced the outcomes of this research. 


\section{CHAPTER FIVE: PHOTOGRAPHS}

In this chapter, I present the photographs taken by the queer youth participants to answer my first research question into understanding Wellington queer youths' experiences of their subjectivities in everyday spaces. Alongside the photographs are the captions created by the participants and the individual discussions of experiences of the spaces portrayed in the images. With the contribution of the participants, the photographs have been thematically and spatially grouped and presented here in three sub-sections: public, semi-public, and private spaces.

\subsection{Public Spaces}

The first section of this chapter is dedicated to presenting photographs of spaces that are located within the 'public' sphere. As outlined by the participants, these spaces are seen to enforce strict heteronormative norms and pressures on their subjectivity. However, as later described, there were opportunities to subvert these pressures when spaces were occupied with certain beings and objects.

\subsubsection{City Streets}

In engaging with their everyday spaces, the participants' most photographed space in the photovoice project was taken while walking through, or interacting with, city streets. In this space, certain elements of the street such as the people, symbols, friends, and animals contributed to a variety of experiences. Further, the queer youths' experiences were also greatly dependent on attributes such as dress sense, gender subjectivity, and mental health.

Like my own personal experience that began this thesis, some of the queer youth said they had been targeted for homophobic and transphobic violence when they have been holding a partner or friend's hand that 'appeared' as the same gender as them. As a result, they would often receive negative verbal responses from people on the street. Charlie told me in the focus group that they had mixed feelings about being on Wellington City's streets, saying:

The only time I've had something homophobic happen to me I was walking home with my friend and we were holding hands and I think it was a homeless man or someone who just started calling us disgusting ‘yuck, yuck' kind of a 
thing, and it just left an unsettling feeling in my stomach. Even before I knew 'what's wrong with this.' But it's good in Wellington now, has come a long way. Nich and I comfortably walk around Wellington together holding hands.

\section{(Charlie, 22, They/Them, Non-binary, Pansexual, Queer)}

Shay also believed that Wellington was a safe place to hold hands, but said that they had an "advantage" over some queer youth of not outwardly presenting as a specific gender. This made it harder for people on the street to tell if they were holding hands with someone of the same gender.

Compass thought there were still some negative feelings towards queer relationships and personhood from people on city streets, saying that holding hands with a partner out in public still makes them feel "uncomfortable".

Taking a self-portrait on Cuba Street (Figure 5), known to be the 'queer street of Wellington', unfortunately led to these uncomfortable experiences happening in the project. As Compass explains:

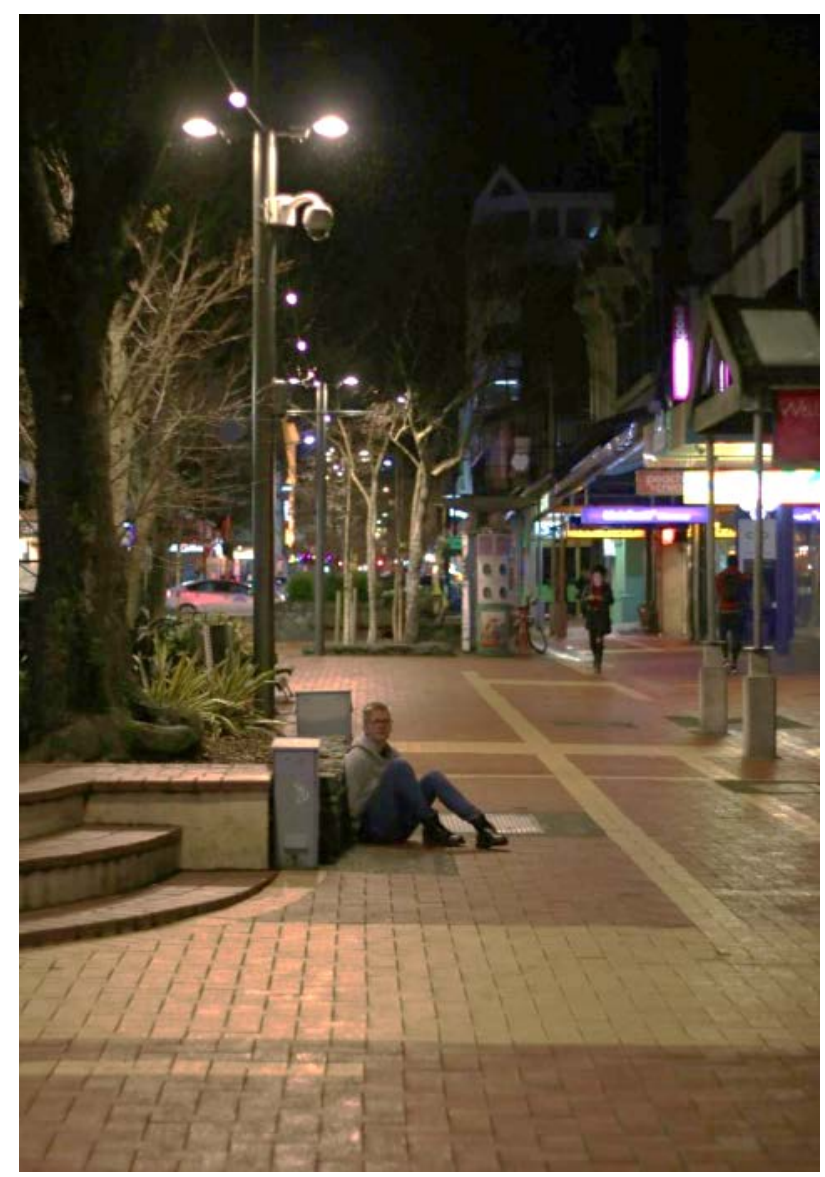

Figure 5 Posey Dyke (Compass) 
... whenever I walk down Cuba Street I always feel like everybody is looking at me and always watching me and always judging me because I feel like, here in Wellington, we have those groups and they are like edgy, but you make eye contact with them and you are like, I know that they are just ripping me apart inside their heads, and actually while I was taking that picture, these two people walked by and they were like 'posey dyke' and I was like, I proved my own point.

\section{(Compass, 17, They/Them, Queer)}

What disappointed Compass the most was their inability to feel comfortable and included within what has been defined by others as a 'queer space'. Compass believed this feeling of exclusion was the case because the street space was occupied by cliques of queer people that often disapproved of Compass and their particular subjectivity and appearance.

Compass explained this further and said in the focus group:

... I don't really associate [Cuba Street] with the kind of queer spaces that I am normally in. The thing is, I do a lot of work with InsideOUT and that, where the spaces are always super open, and everybody is there and everybody cares, and everybody knows about mental health, and everybody is like 'Dress how you want, be who you want, you look great, just do you', and I feel like Cuba Street is that like that other side, where it kind of feels like that toxic side of our queer community, where like it's kind of like everybody is trying to be edgy for face value, but nobody like really gives a shit and yeah

... in our queer community there are still assumptions even if they are different assumptions [to heterosexuals]. If you are non-binary then you must look a certain way or you must dress in a certain way and that ... It seems like it should be a really inclusive queer space, but also like it doesn't encourage you to be your own individual or like, it just feels really claustrophobic.

\section{(Compass, 17, They/Them, Queer)}

One further photograph and experience detailing an example of the intra-community challenges experienced by queer youth on city streets is Kate's photograph shown in Figure 6. This photograph represented an instance where imagery, symbols, and iconography seen on a city street from within the queer community was not necessarily 
an inclusive one. Kate's image of the particular poster was later found to be from a group of Trans-Exclusionary Radical Feminists (TERF) ${ }^{8}$ in Wellington. Initially Kate took the photograph believing it was a positive and reaffirming message about their queer subjectivity on the city street, but was later shocked to find out the subliminal messaging of what was trying to be portrayed via the poster.

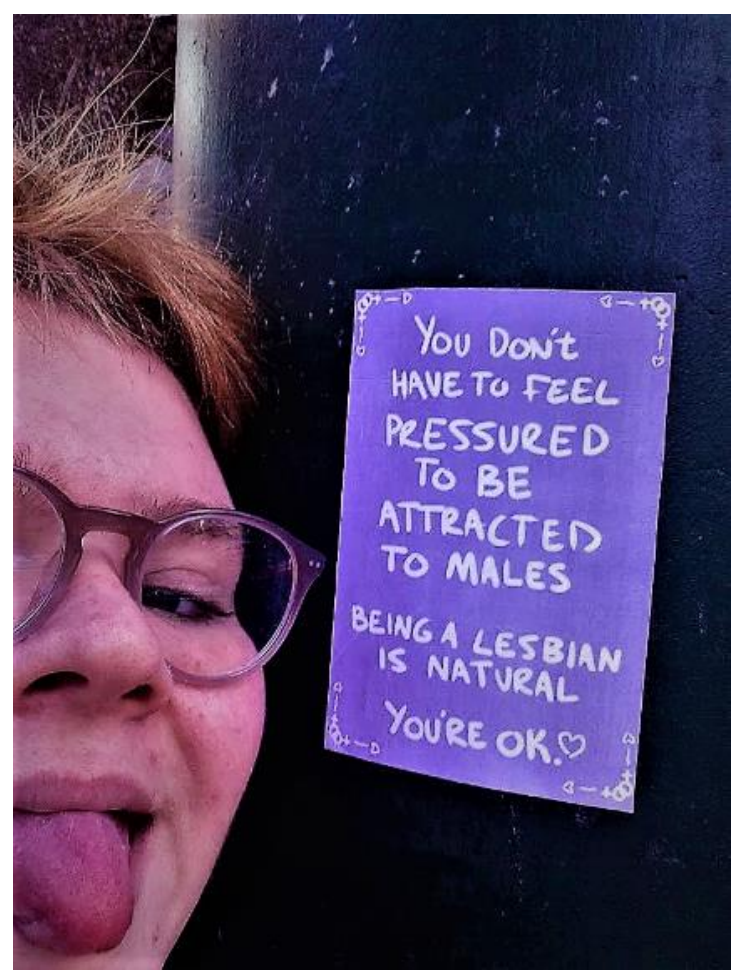

Figure 6 TERF Poster (Kate)

Kate reflected back on a previous experience with the TERF community after taking the photograph and remembered when the group recently protested at Parliament against inclusive gender and sexuality options on the upcoming census. They explained how this group's beliefs can have effect on non-binary and trans people:

... they have been saying like: 'Women don’t have penises' or 'Women have vaginas'. All this kind of stuff which is also, as I realised, is very difficult for non-binary people because I keep seeing all this stuff that says 'Women have vaginas' and I'm like cool, yeah, that's shitty of you to say, wait, you're talking

\footnotetext{
${ }^{8}$ Trans-Exclusionary Radical Feminists (TERF) is used to describe a branch of feminism that opposes the inclusion of trans women into debates relating to cis-gendered women and often dispute that trans women are women.
} 
about me and like that also is a realisation that I am being grouped in with these people and these beliefs, which is like a weird thing I am still processing 'cause I think we kind of assume the 'other'. We don’t think about our own personal connection to things.

\section{(Kate, 20, They/Them, Non-binary, Queer)}

Kate and Compass's photographs and experiences showing negative imagery and comments on the street space highlighted that even within the queer community there can be negative, bigoted, and exclusionary views of other queer subjectivities.

However, there were other instances when queer imagery, symbolism, and iconography contribute to a positivity feeling of subjectivity and openness on the street. In relation to Figure 7, Kate explained the joy of finding the Keith Haring sticker shown and said:

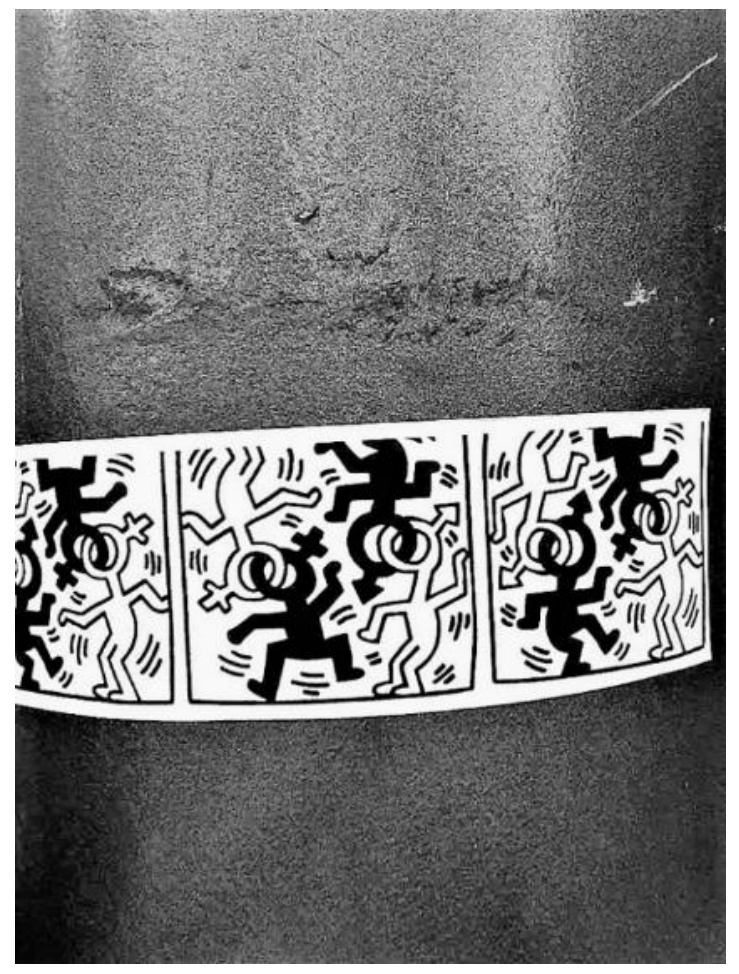

Figure 7 Symbols in the streets of Wellington (Kate)

... it is really really rare to see something like explicitly affirming queer identities in public spaces on the street ... I rarely feel affirmed in that kind of way in public 
... it made me so happy because I am such a Keith Haring fan and he just did such cool art and was just, I don’t know, he was really significant and we see this iconography like everywhere and just to kind of have it on the street in Wellington, and someone had like decided to put it up. I am like 'That is really cool!' and it's, I guess, that kind of queering of normal spaces, you know, symbols and images and representation.

\section{(Kate, 20, They/Them, Non-binary, Queer)}

They went on to say that what made it special was the fact that the sticker and imagery was not isolated, nor was it present within a queer space, but it was in and amongst every other sticker and poster on the pole on the street. Kate proudly stated:

Obviously there is a thing with queerness about trying to be hegemonic and fit in and like obviously [this] in itself is a problem, but with this [sticker] it doesn't feel like that, it just feels it is just there. Separating it would be weird, so it was cool to see it amongst other posters and see lots of them around town.

(Kate, 20, They/Them, Non-binary, Queer - Kate’s emphasis)

In the very same 'queer space’ that Compass experienced being singled out for being different, Compass also took a photograph of a traffic light design representing a Carmen Rupe silhouette ${ }^{9}$ - one of the most celebrated queer pioneers and entertainers in New Zealand (Figure 8). She was memorialised in these pedestrian crossing lights for the $30^{\text {th }}$ anniversary of the Homosexual Law Reform Act 1986 (Townsend, 2018).

For Compass, seeing this crossing lights compensated somewhat for the negative feelings of the street since having this queer recognition in very public spaces is important, even if they are in a space that they do not personally enjoy. The ability to recognise and relate to visible imagery and symbolism of queer personhood in this space gave Compass an overwhelming sense of pride. The photograph itself was taken to centre the bright light of Carmen in the photograph and to focus our attention away from everything else on the street; mimicking the same experiences by Compass.

\footnotetext{
${ }^{9}$ There is not enough words to detail the fabulous life of Carmen Rupe in this thesis, including that of her activist work, her attempt at Wellington mayor candidacy, and management of sex work establishments. See Lynette Townsend's piece in the Te Ara online New Zealand Encyclopaedia for a detailed biography.
} 


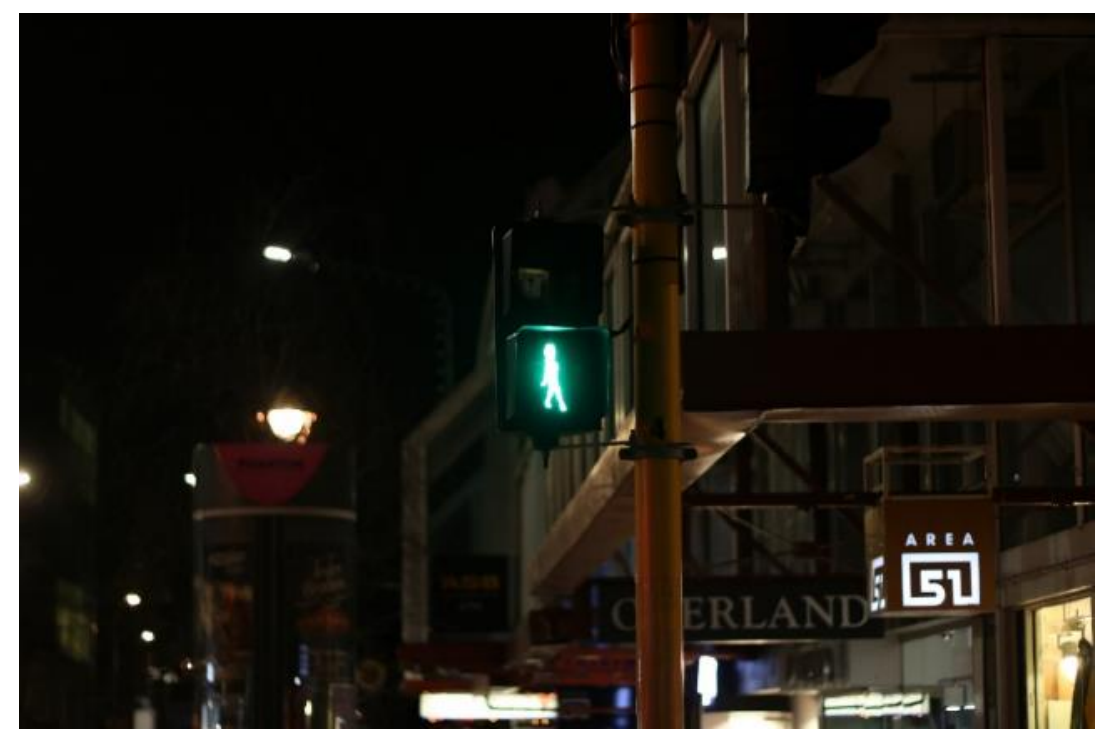

Figure 8 Carmen (Compass)

Lillian's photograph (Figure 9) further reflected the importance of signage and visual representation for feelings of safety and belonging on the city street. Lillian said that by seeing the rainbow flag on a café window she knew that she had allies in that shop, and that if she entered into that space she could be herself. Although not a common feature of many spaces Lillian engages with, she said that she appreciates small actions of recognition that help queer people tell if a space is inclusive of their queer subjectivity:

I hadn’t noticed this sticker until I had walked past it, so of course I wanted to take a photo, it's just like a little reminder that somebody, I am like 'ah okay, I have allies in that shop that's a cool thing to know' ... and it's like 'aww thanks for that public support'.

\section{(Lillian, 16, She/Her, Female, Queer)}

The same rainbow flag also became a symbol of further pride, but also discomfort, when Lillian decided to wear it around her body as she walked down the city street (Figure 10). She said:

... it was a weird experience because it felt really nice wearing [the rainbow flag] and going on public transport and what have you, but at the same time I was constantly checking to see if people were looking at me or had acknowledged me or were reacting to the flag in any way. So it felt good, it 
was a symbol of pride, but I don't think I have ever been more hyper aware of myself going through a public space.

\section{(Lillian, 16, She/Her, Female, Queer)}

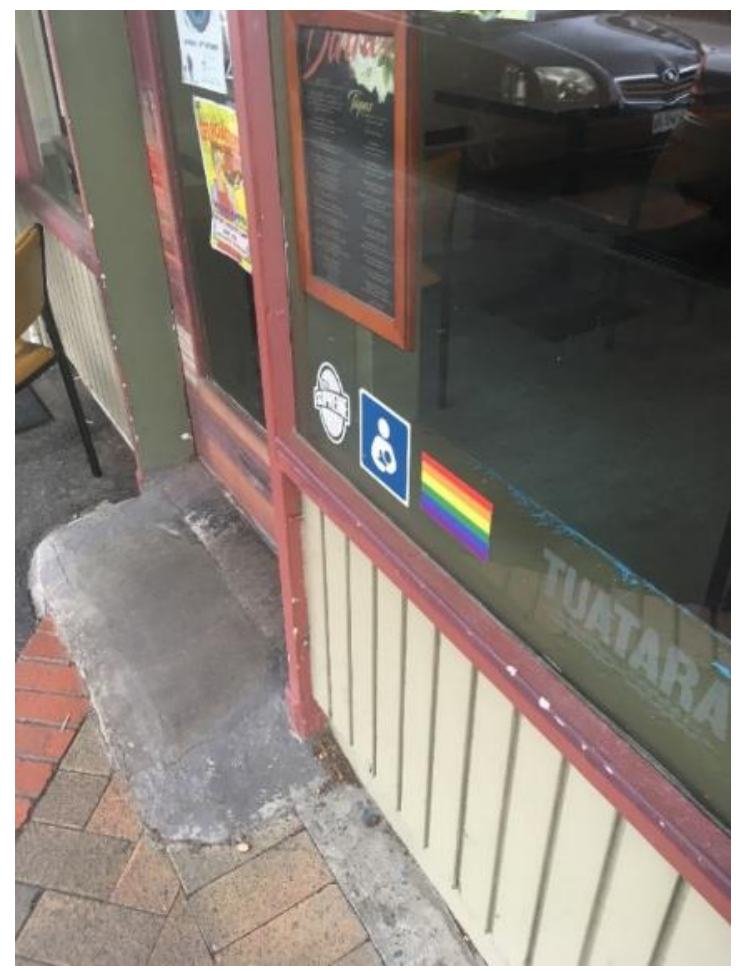

Figure 9 Rainbow flag in Cafe Window (Lillian)

She explained to me that this was not normally the reaction she would get when walking down the street. Lillian said that she could often be perceived as being queer by the way she dresses, but found the attention she received because of it was as a positive thing. She added:

I definitely like I have a fashion sense that most people would associate with the queer community or you know, or would attract women, that whole young sort of teenager fashion sense, I know that I have that and that's something I embrace because best case scenario it attracts those other people to me and it's like a good way to find the gay-dar, it's pretty much real and it's something that is useful, honestly.

\section{(Lillian, 16, She/Her, Female, Queer)}




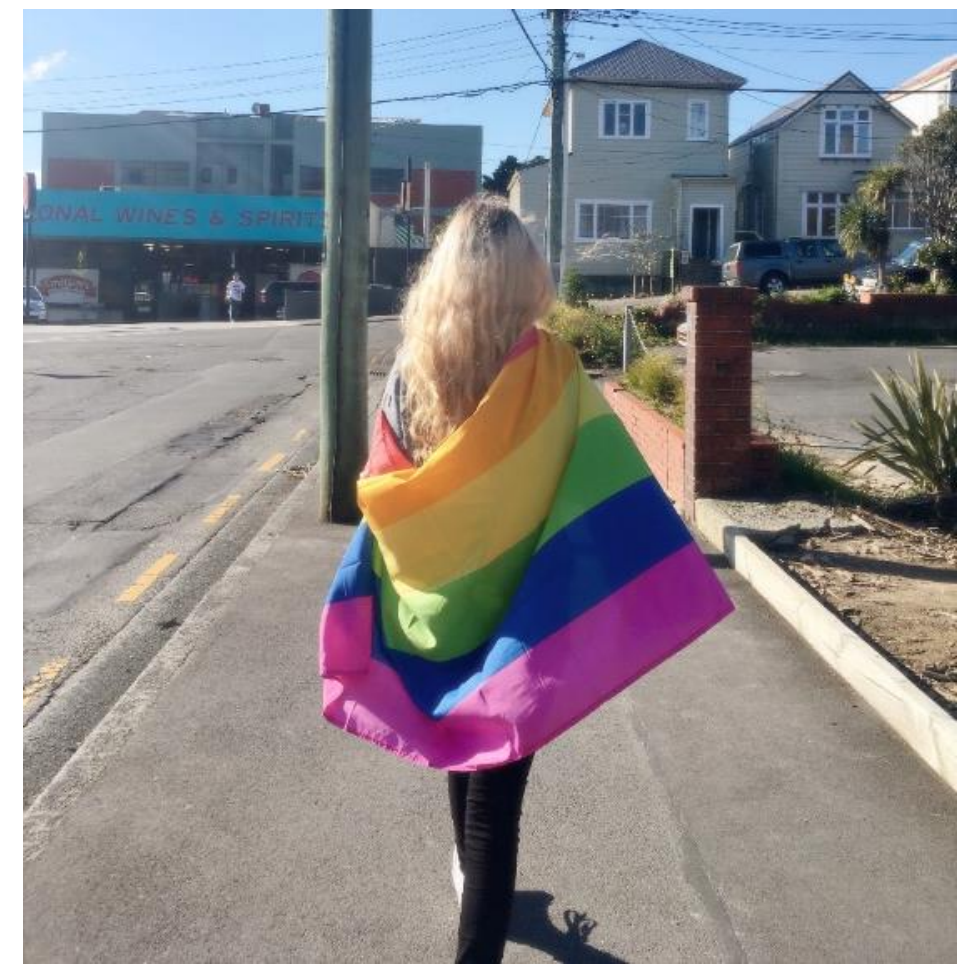

Figure 10 Rainbow flag on City Streets (Lillian)

One important aspect that Lillian, Shay, Kate, and Compass all spoke about was how city streets' (and other spaces') physical elements is not always what makes it inclusive, open, or safe. Often it was dependent on the people or beings that were in the space. Kate said:

... for me mostly it is about people, this [interview meeting room] for example feels like a queer space cause we are together and I think it doesn't have to be necessarily about visual markers or symbols or words, it can also be about feelings.

(Kate, 20, They/Them, Non-binary, Queer)

Similarly, Lillian said being with someone that shared a common subjectivity made every space they walked through safe and comforting.

Although experiencing challenges with their subjectivity on city streets, one of Compass's safest spaces was shared with their friends in a pedestrian tunnel on the street (Figure 11). Speaking to the experience, Compass said:

... it was a place that was super special to me, and me and my friends use to go to that tunnel because we were like, you know, when you just had a long day and you're a rebellious teenager so you'd leave the house at one in the 
morning and then you would go to a tunnel and it was just like, some really good times. I think, that was definitely during a period of my life where I was kind of like, 'I don't know what's up' and you know, kind of trying to do that and my friends were a really big part of that.

... that is kind of like a feeling I like to go back to. That is a feeling my friends give me quite a lot, especially since my coming out and before then they gave me a lot of comfort in that knowing that even though I might not feel initially comfortable, they supported me through that and they would, you know, respect me and all aspects of who I was.

\section{(Compass, 17, They/Them, Queer)}

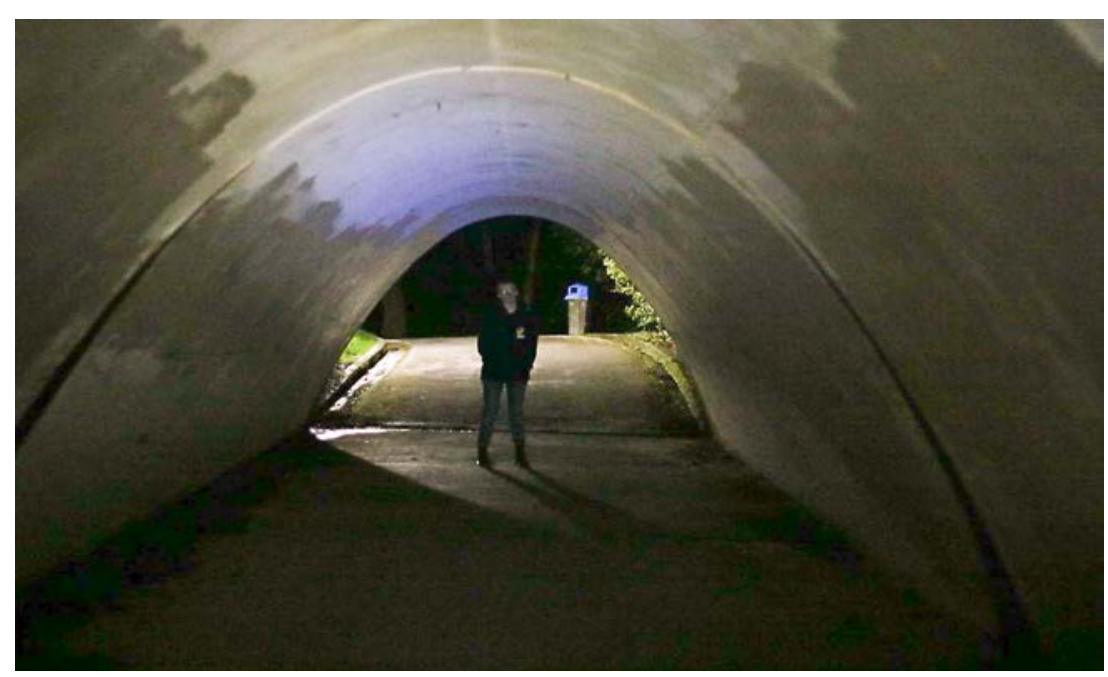

Figure 11 Having friends that are supportive are the most precious thing (Compass)

Much like friends, animals provided the same feeling of comfort, safety, and stability to Shay, Lillian, and Nich and were either photographed or discussed in their interviews and the focus group.

For these participants, animals acted as a bridge between safe and unsafe spaces, in particular on the city street. The reason that queer youth often made connections with animals is because they are themselves not pressured by heteronormative discourse and are able to give comfort and safety wherever they may be. Lillian spoke at length about how animals are a positive being in their life and said: 
... [the cat] doesn't know what homophobia is. I mean I am pretty sure I had the same reasoning as other people in the project [to take photographs of pets], which was like animals will understand. Animals can feel your sympathy, feel your pain, and can often sympathise with it in a way that doesn't involve like a human-society-kind-of-thing. They just know that you're a person and you're upset and they want to make you feel better. They don't know why you are upset and they just kind of want to be there. It's simple I guess. It about forgetting about things for a while with an animal.

\section{(Lillian, 16, She/Her, Female, Queer)}

Shay expressed an emotional connection to animals through their photographs (Figure 12) saying that the past few months had been especially impactful on their health, and when they interact with dogs, either on the street (which they often found constricting) or at home, it brightens their day.
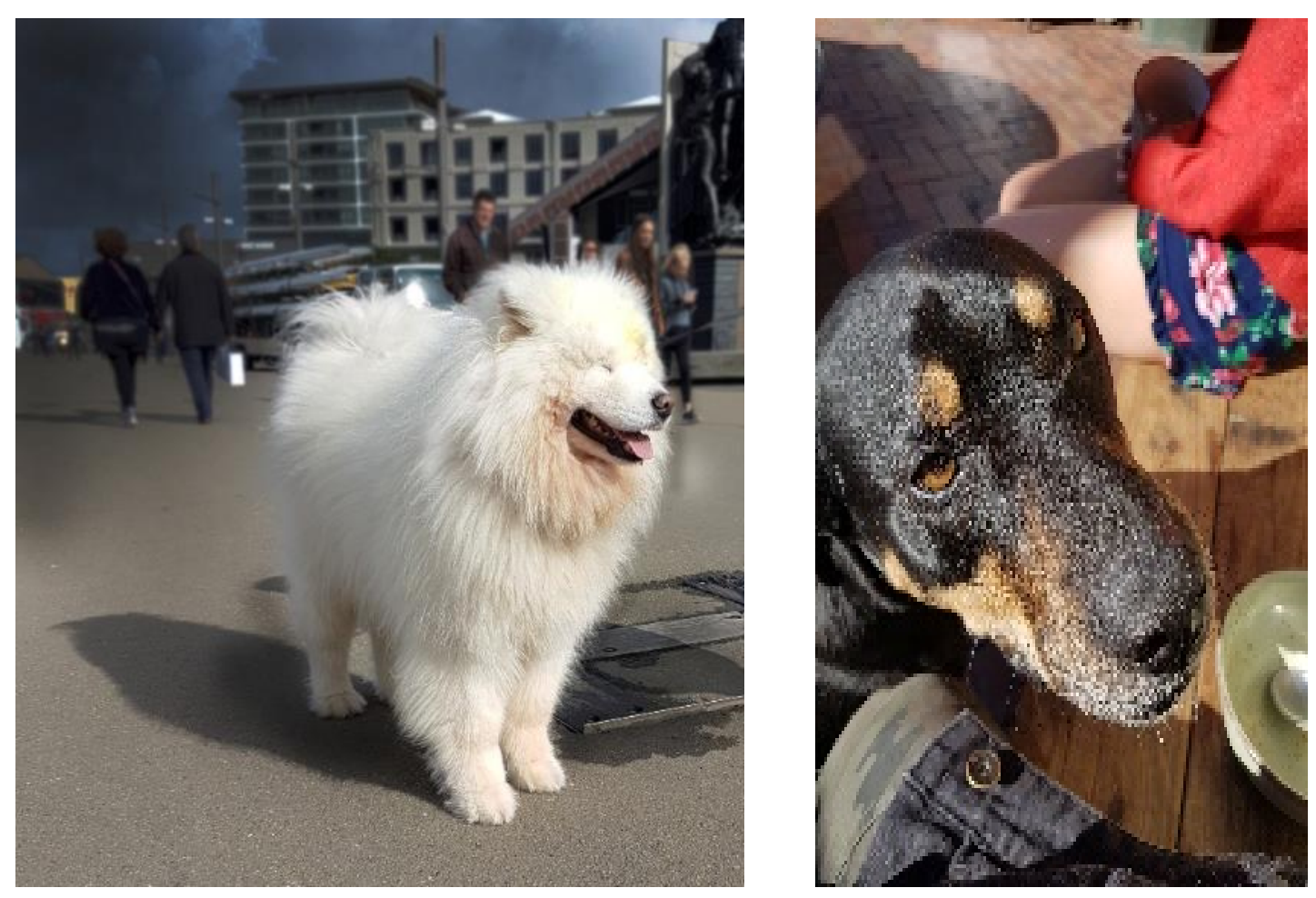

Figure 12 A dog does not care what you identify as (Shay)

Shay said:

... animals either love me or hate me, but it's not for the same reasons as humans, it's more 'stranger, curiosity, food' and it's refreshing ... 
... the fact that all the pressures of everything in life don't matter suddenly. It's more about being in the moment, and feeling something instead of worrying about everything else. Dogs don’t give a damn.

\section{(Shay, 20, They/Them, Queer)}

Shay believed that through animals and other objects, they can transcend reality and escape away to a space with less pressure:

I prefer being able to live in a different place and sometimes it's through something like an animal, sometimes it's through something like a book, sometimes it's something like a landscape or a view.

\section{(Shay, 20, They/Them, Queer)}

Talking to me about their photograph of their dog Robbie (Figure 13), Nich said that their dog was calm, peaceful, and “loves everyone”. Like Lillian and Shay's comments, Nich felt Robbie saw everyone as a friend regardless of what they identify as. Nich also appreciated how walking Robbie helped them to engage with the outside world, made them feel calm and comfortable walking on the city street, and got them out of the house and into one of their favourite places in the city - the waterfront.

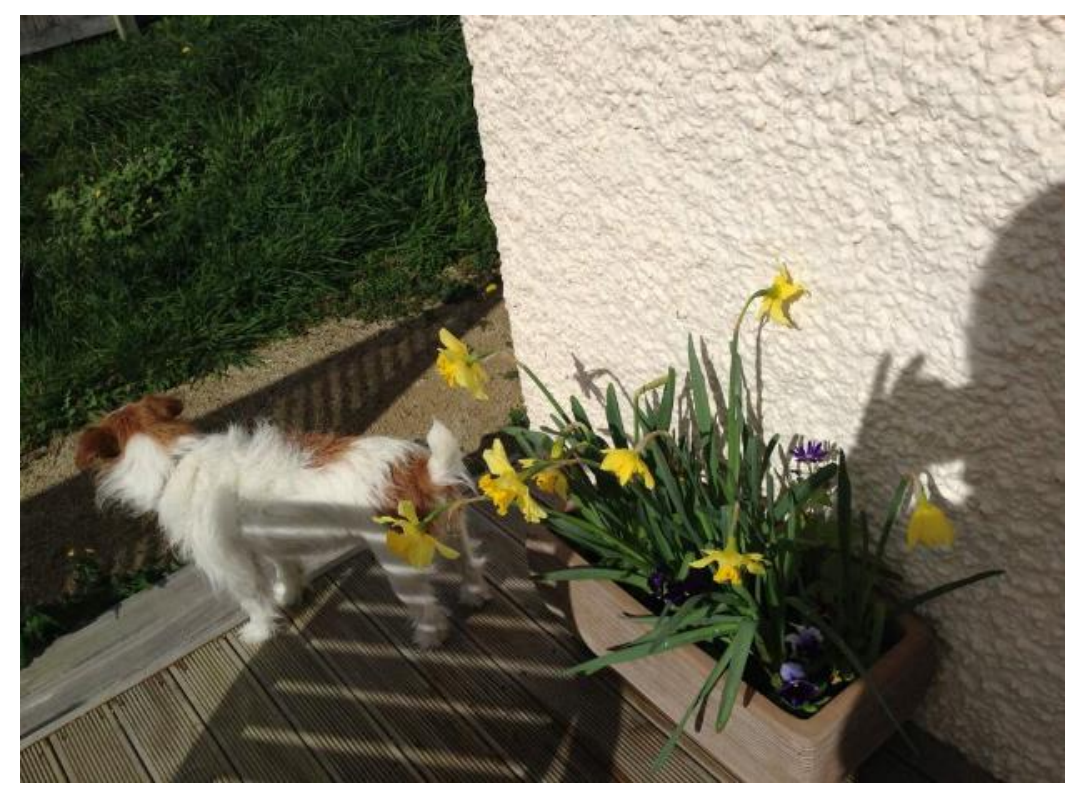

Figure 13 For my dog 'my dog Robbie I can always be comfortable with, he is never judgemental! He's so happy with being alive and hanging out!' (Nich) 


\subsubsection{Public Transport}

In similar regards to city streets, photographs and group discussions on the experience of public transport was brought up as a space that often evoked negative and conflicting experiences.

Charlie took a series of photographs (of which one is displayed as Figure 14), from inside a bus on their way into Wellington. I asked Charlie of their experience of the bus and why the photograph was shot looking outside, and they said:

I do tend to take window seats, as you can tell from where the pictures are taken. It's just a personal thing where I prefer to kind of be in a corner, so in this case I choose a window seat because it's closest, you know. I also just like looking out and seeing things go by.

(Charlie, 22, They/Them, Non-binary, Pansexual, Queer)

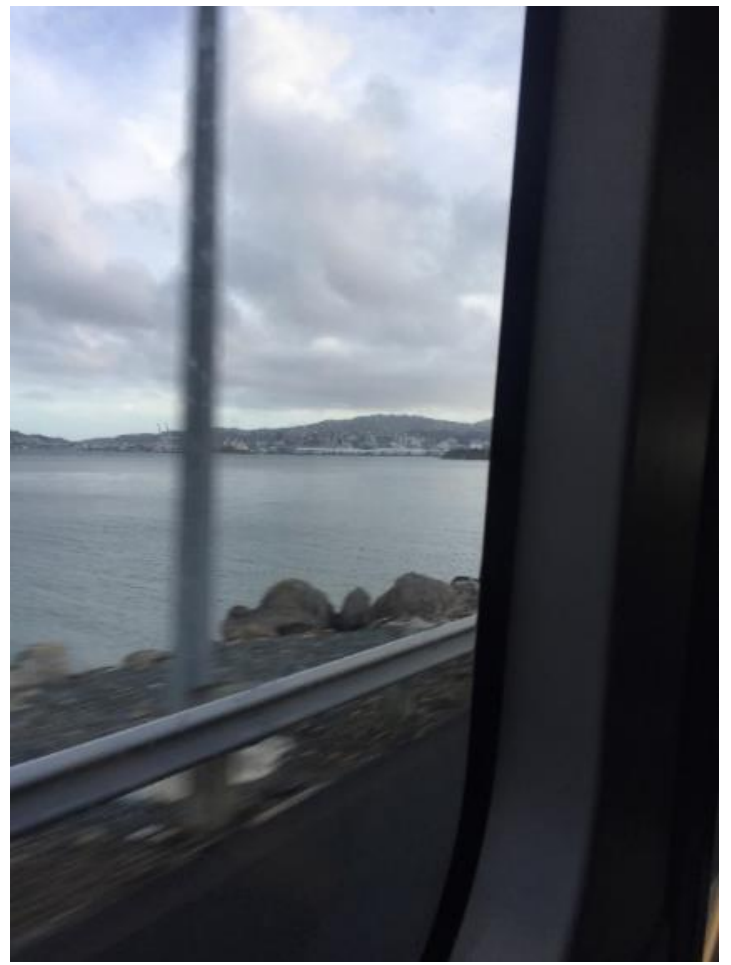

Figure 14 Local gay on the bus (Charlie)

Charlie said that the bus can often feel claustrophobic especially when it begins to fill with "mainly cis-heterosexuals" and that by sitting near the window they can ignore the small talk and the 'hi ma'ams' and focus on the nature and beauty outside of the bus. 
Similar to Charlie, Shay believed that the inside of the bus symbolised the mundane 'nine-to-five' living that is dominant in society. They saw the people on the bus as grumpy and frustrated, with no personality and conforming to "the normal”. Shay said that they do not want to be that way, saying that the darkness that is seen in their photograph (Figure 15) is:

... a mixture of things, it's in a sense conformity and pressure of the world. I am well aware that there's so many pressures especially that these people here represent when they are grumpy and everything, and it's like that's what you are going to become, but when I look at this I don't need to become that, I could become something bigger, brighter than that.

\section{(Shay, 20, They/Them, Queer)}

Shay said that the inside of the bus felt claustrophobic and constricted and was a restrictive space. For them the space did not give the ability for individual expression. There were clear rules and regulations about how to behave on the bus - how to look, act, when to speak, engage with the person next to you.

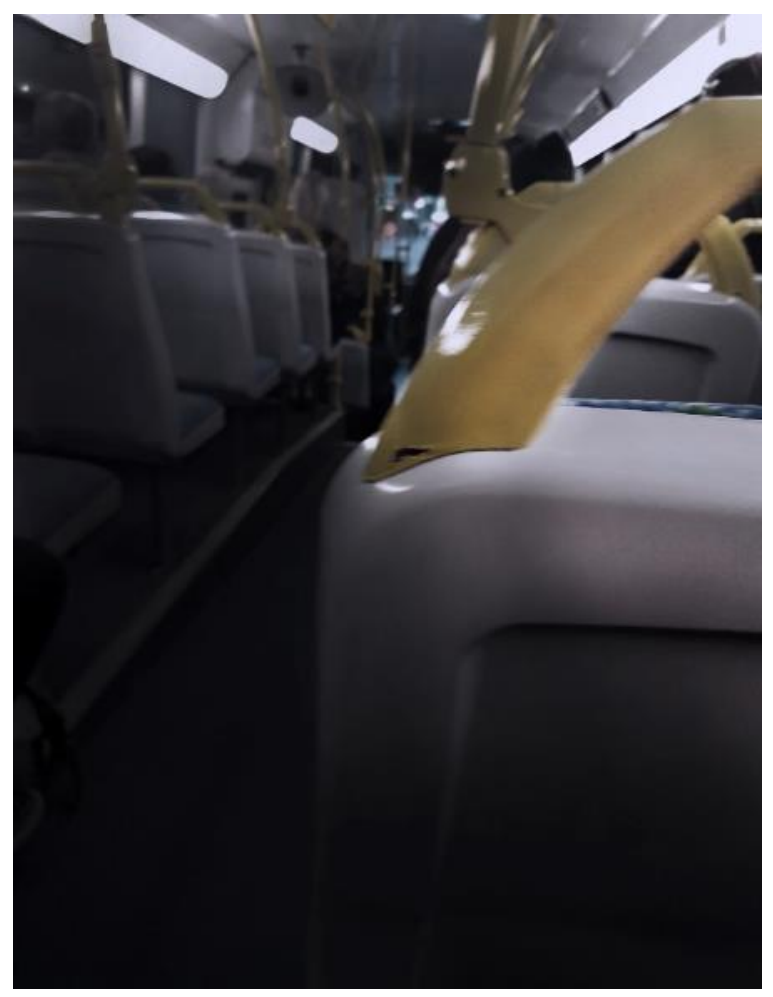

Figure 15 On the bus (Shay) 


\subsection{Semi-Public Spaces}

The second section of this chapter presents participants' photographs of semi-public spaces. Within these spaces, the normality and regulation of heteronormativity begins to blur with participants' ability to challenge gendered and sexed space. However, theses spaces still have strong expectations on appropriate behaviour, dress, and subjectivity and contribute to instances of negative experiences of space for queer youth.

\subsubsection{Nature}

Natural spaces for participants encompassed green spaces, oceans, beaches, trees, and landscapes. In such spaces, queer youth interacted with nature either on an everyday basis or saw it as a space to escape away from the stresses of everyday life.

For Compass, the latter is true. For them, they saw the local park near where they live as a space that provided an opportunity to remove themselves from the pressures of the world and to reconnect and recharge (Figure 16). They said of their feeling in the moment, that they needed to "sit back and find somewhere that is quiet, so I pulled up there and just sat there and I was like, I feel good, I feel better”.

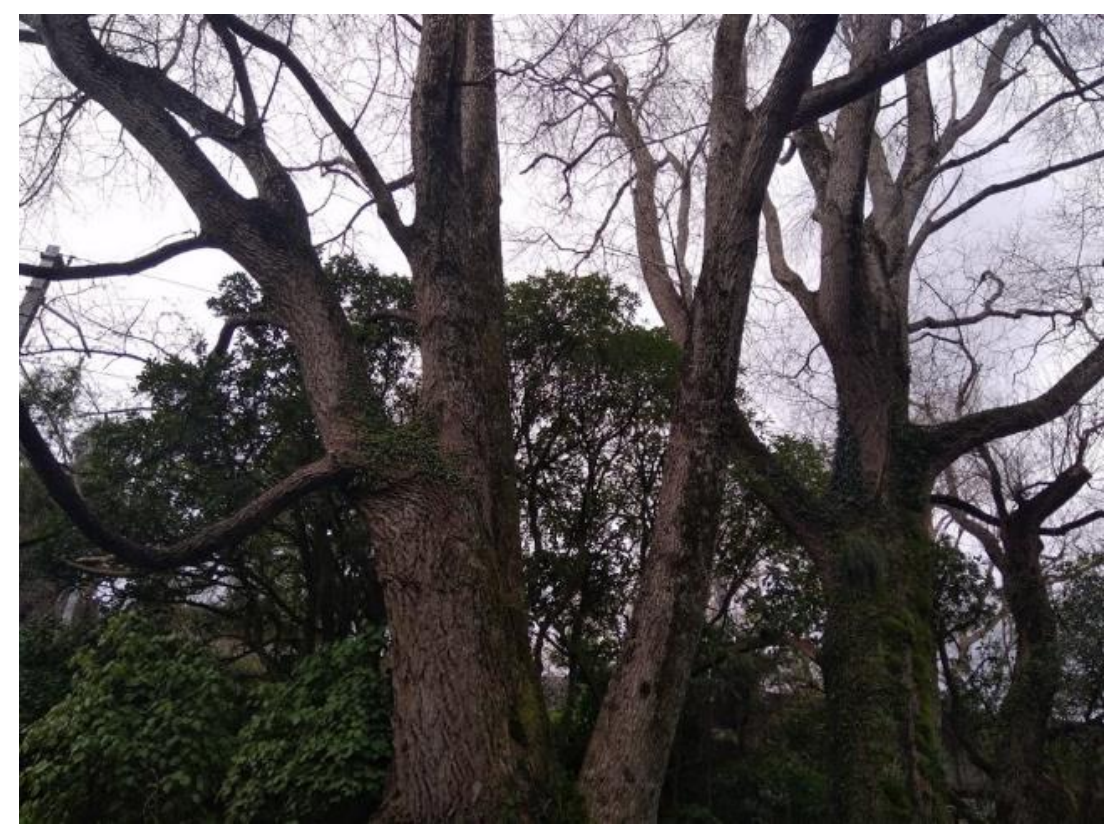

Figure 16 It's important to get away from the homophobes (Compass) 
Similarly for Charlie, they saw the beach as peaceful and relaxing, associating it with family, Christmas time, and somewhere to go when there is nice weather (Figure 17). When discussing the photograph, Charlie said:

Everyone is doing their own thing. It's away from the city where there is less people, maybe there is less scrutiny. You can feel like you're by yourself here and one with nature.

(Charlie, 22, They/Them, Non-binary, Pansexual, Queer)

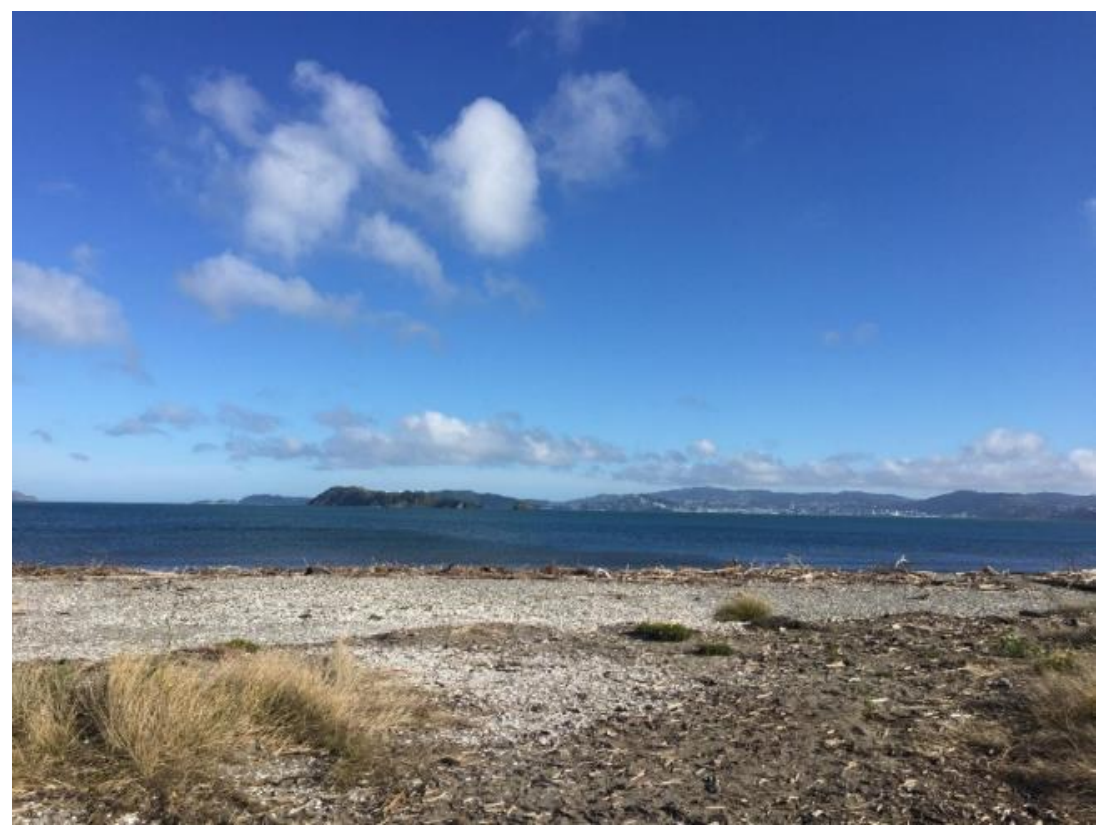

Figure 17 Gays at the sea (Charlie)

On the other hand, nature was also a routine part of many queer youths' everyday spaces. Nich conveyed the importance of observing the beauty of nature in daily life like passing the time when waiting for the bus or "being in the moment" while going out for walks in their two photographs in Figure 18 and Figure 19:

I just love observing little bits of nature even though it's just on your daily life kind of stuff. Even if I am in town and I'm like 'oh dang there's a cute little pigeon' ... or if there's a random butterfly, I think that's one of my favourite moments - you're just walking out and about minding your business, then a butterfly comes along.

... just to look at something and be like 'wow that's just dang pretty, let me remember that' I just love a good sky. And then, walking with my dog just by 
myself. I just like going for a nice little quiet walk and then, just kind of thinking to yourself - a lot of the time if I'm by myself I do listen to music but I also do it without headphones to reflect on stuff and think about whatever I'm thinking about.

(Nich, 22, They/Them, Pansexual, Queer)

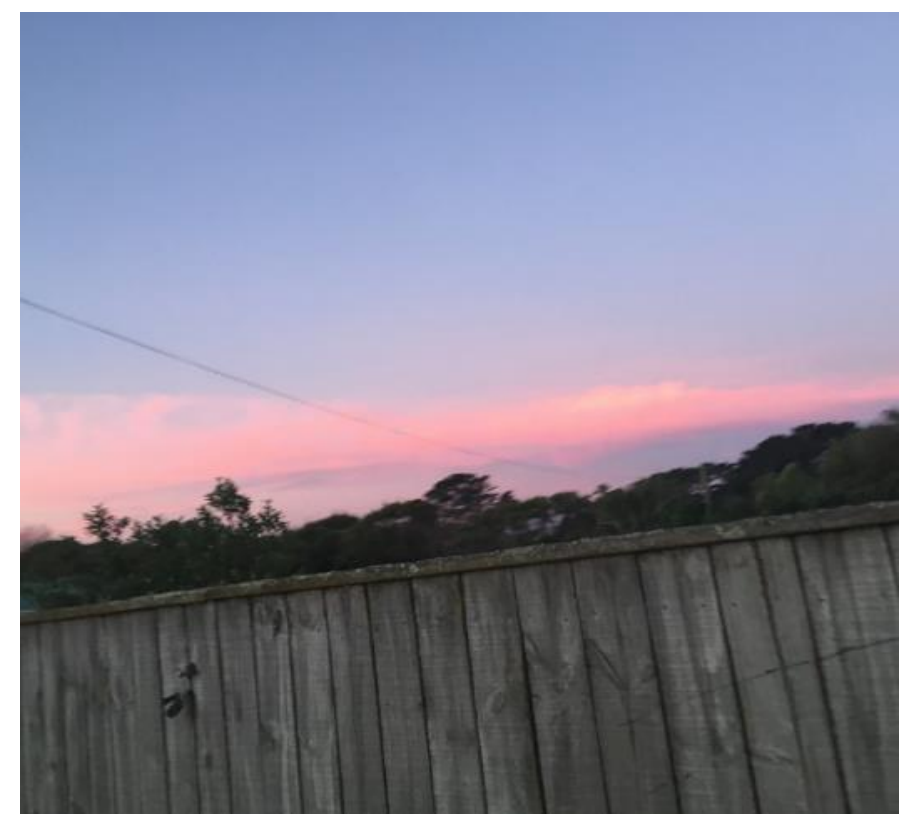

Figure 18 I just love looking at the sky, it's so pretty all the time! I feel peaceful looking at it. The sky isn't judging me for my sexuality, it's just there above us being cute! (Nich)

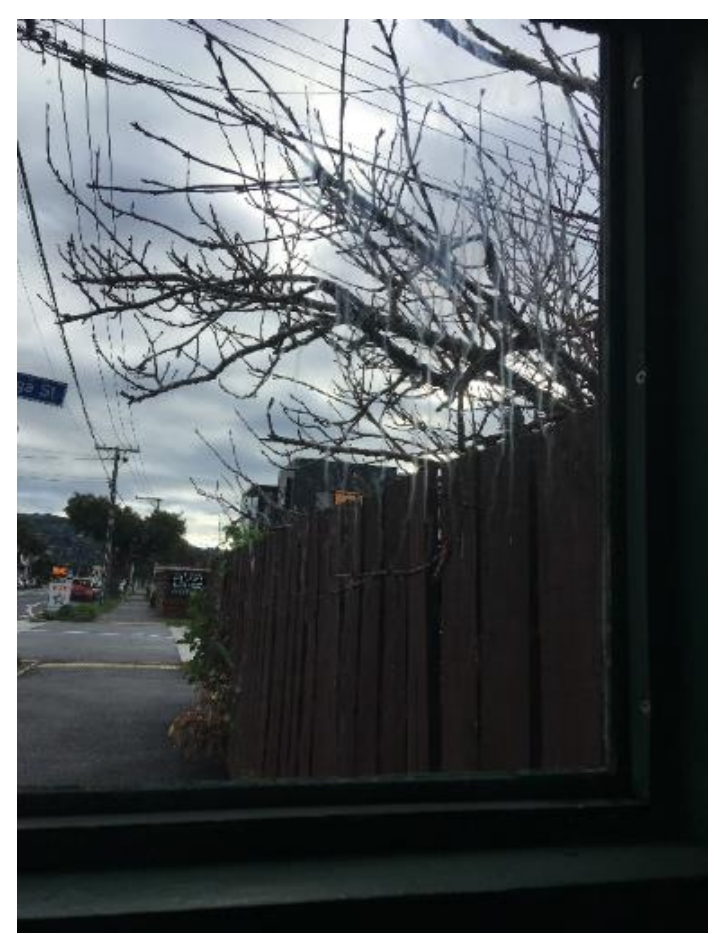

Figure 19 Taking the Time to Appreciate Nature (Nich) 
There was also significance of enjoying landscape views for Shay when they took a photograph overlooking the city from atop a hill, and another of the city waterfront (Figure 20 and Figure 21). For them, like for Charlie and Nich, being out in nature enabled Shay to be removed from the mundane and repetitive drain of day-to-day life. Shay says that these photographs represent:

Peacefulness. I have always felt more peaceful around heights and views and I have always loved being in cities at night time because I find especially when there's a height or something like an ocean and you can see a whole bunch of lights, it’s refreshing, it fills me with energy more positively than everyday life.

(Shay, 20, They/Them, Queer)

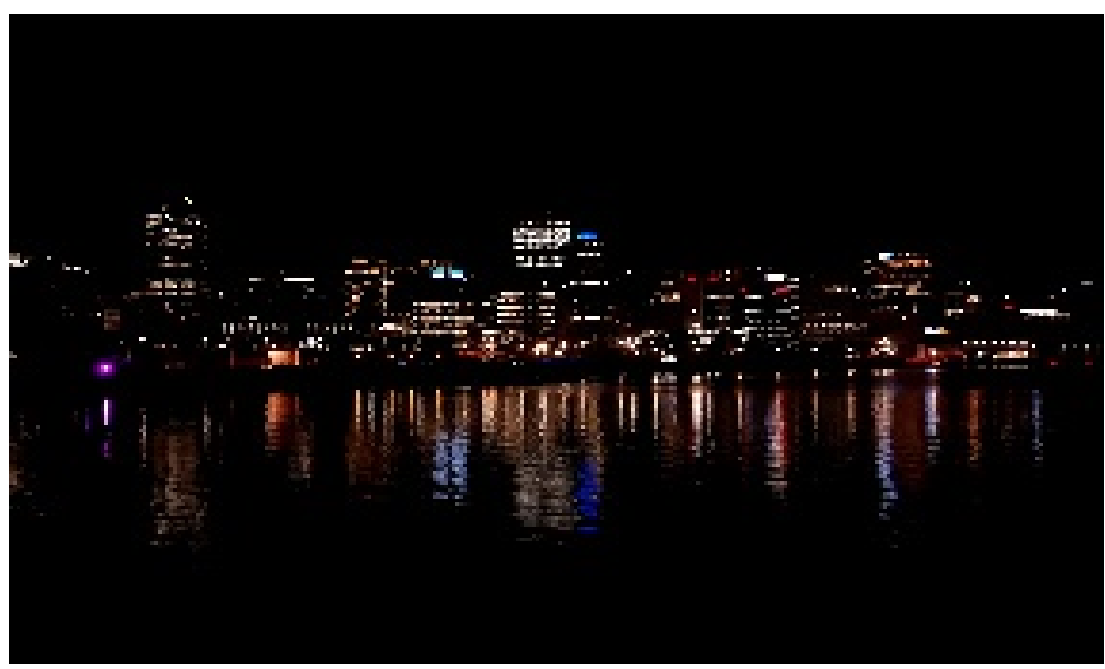

Figure 20 In Darkness there is Light (Shay)

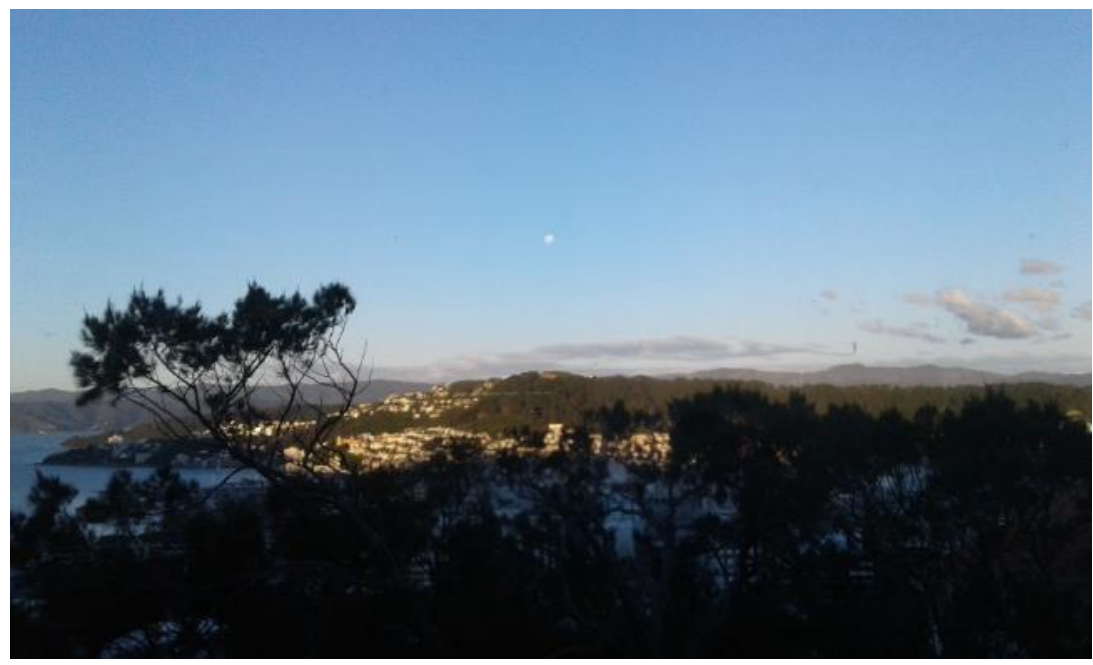

Figure 21 Over the Campus (Shay) 
The contrasting day and night photographs Shay presented in the project show two different atmospheres: The view from the city was refreshing and provided them with more positive energy than their other everyday spaces; while the nightscape, portrayed Shay's ability to find the metaphorical 'light' in the 'darkest' of places (represented by the twinkling lights of the night-time city).

\subsubsection{School}

Within the schooling space, participants shared stories of both positive and negative experiences of the space. Accepting teachers and students, pride festivals, and student groups provided many of the queer youth with experiences that contributed to their feelings of safety, inclusion, and positivity. However, institutional and historic norms also contributed greatly to the negative experience of the schooling space.

As an active and lead member of their QSA, Lillian took numerous photographs of school spaces around the time of their pride week, showing pride flags, banners, streamers, and the events which she organised. The first event that opened pride week was a drag show in the school hall, shown from behind the stage curtain in Figure 22.

Lillian said the event was the most popular event of the week. The hall reached capacity before the event had started and they had to remove people due to overcrowding. She proudly said that all the performers got standing ovations while students and teachers were approaching Lillian to congratulate her on a successful show. However, as Lillian explained, there were some procedural hurdles she had to overcome to let the event go ahead:

So for this one, the process was a little bit tricky, 'cause I organised this with one of the senior staff ... and she made it clear that we had to be really really careful and conservative with organising this first event because, worst case scenario, if it were to get to the media or something and one of the performers had done something sexual or adult or just any way inappropriate and somebody got wind of that, if one of the girls were filming it and put it on social media then it could be taken and blown out of proportion and we wouldn't be able to maintain relationship with the [drag group] who are the ones we invited, and you know, actually pride week itself might of come under fire if this were to like I guess, 'get out' - which feels awful saying - and be misinterpreted. So we had to be careful, we had to like cover all the windows 
that went into the hall, 'cause you know, 'Mrs' was like we have to be, regardless of how much we want a progressive society, we've got to be respectful of where people's mind-sets are right now if we want to maintain this, so we had to cover the windows again so anybody that would be offended by it, for like religious or moral purposes, wouldn't have to see it.

\section{(Lillian, 16, She/Her, Female, Queer)}

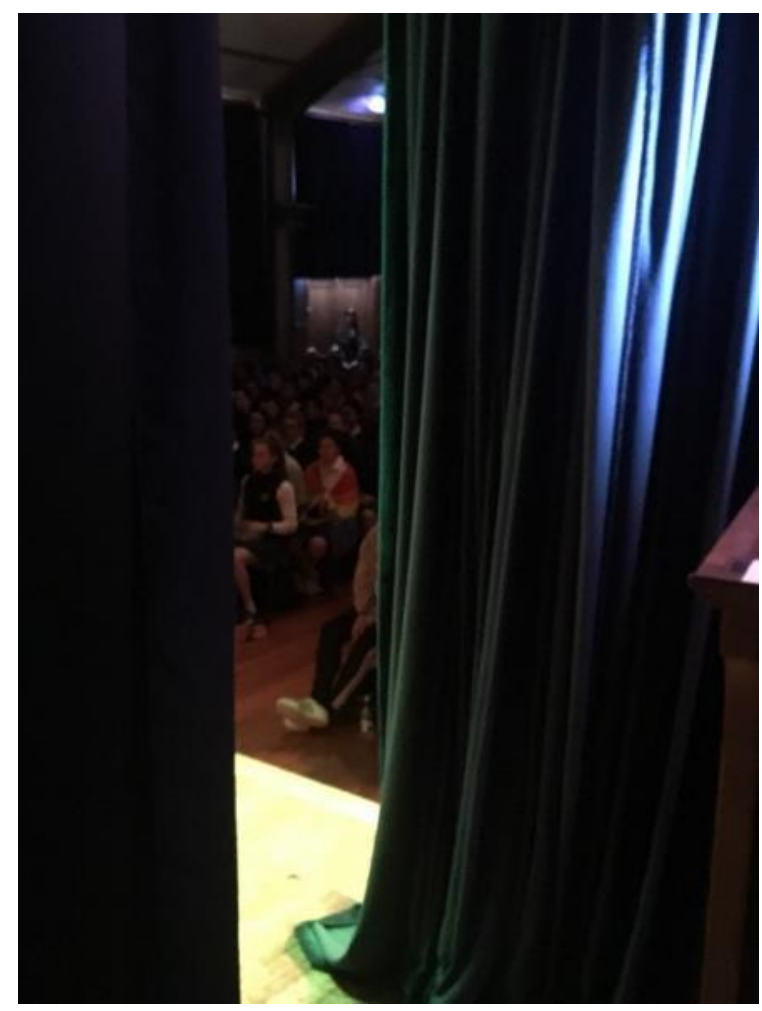

Figure 22 From Behind the Drag Curtain (Lillian)

The strong restrictions placed on the event to maintain it as a potentially long-standing tradition of the school's pride week also included censoring drag performers names, applying dress codes, and limiting advertisements around the school. As Lillian shared, although she was not particularly happy with the arrangements put in place, she was willing to implement these if the event could remain a regular feature of pride week. She mentioned that the event was "a small-step” in the right direction and that hopefully in the future these restrictions could be removed to make the space more welcoming and inclusive. 
The rest of pride week involved smaller events like bracelet making, increasing awareness to the use of gender-neutral pronouns, and decorating the school with pride colours - as can be seen in Figure 23.
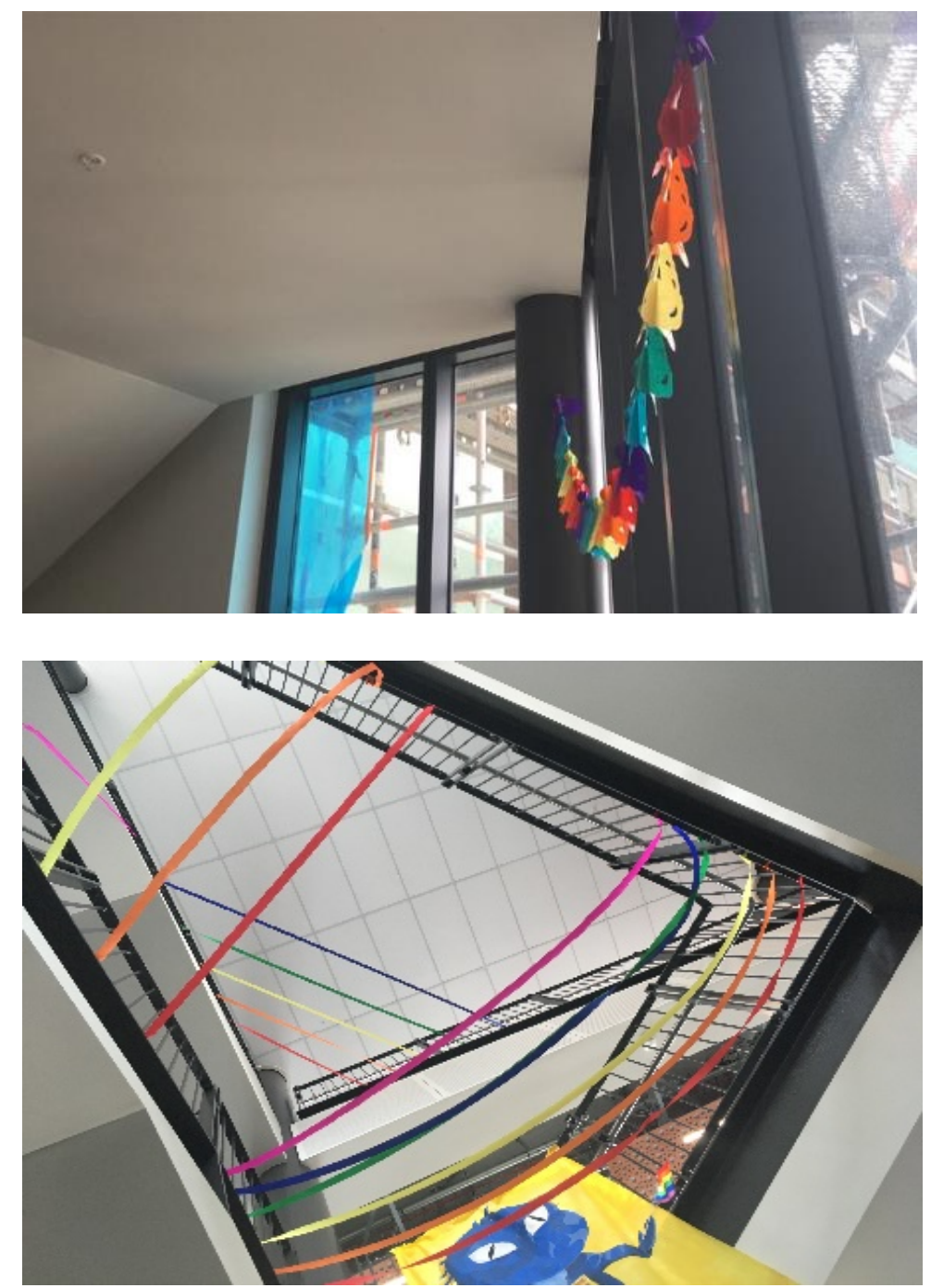

Figure 23 Pride at School (Lillian)

Lillian said that it was very empowering to see such a visible display of pride while also rewarding to see cis-gendered heterosexuals engage with the queer material and posters around the school. For Lillian, one of the most important things for them about pride week is the increased visibility of queer subjectivity around the school space. They said, “... it’s something to be celebrated, it’s cool being part of a community like this you know, it's something to take pride in, so why shouldn't we celebrate that?” Lillian mentioned that the reason events like this were a big success is because the school and teaching environment allowed it. She said that the teachers are hugely supportive of pride week, and eager to participate in the activities. She said she was 
fortunate to be in a school space that allowed such diversity in thoughts, people, and experiences.

Participants also took photographs of their queer students' social spaces and QSA meeting rooms in recognition of the safety and comfort they provided within the school. Lillian's photograph shows the entrance way to room 66, the location of their QSA lunchtime meeting space (Figure 24).

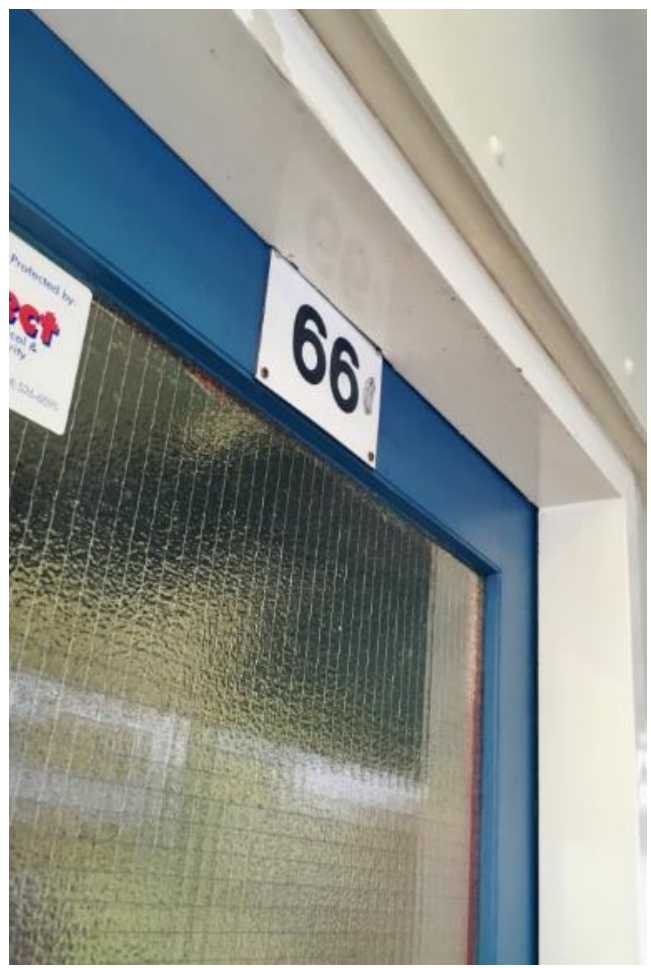

Figure 24 Room 66 (Lillian)

Lillian included this photograph in the project because it was an important space in their everyday life. It was the space that she helped set up and that will continue to be used as a safe retreat for queer students after she leaves the school. It is a symbol of her hard work, determination, and the fond memories she has with her friends and fellow queer students. It is a point of pride and of comfort for her that the picture can be shown and recognised in the project.

Outside of pride festivals and recognised queer spaces within the school, there were mixed feelings about the queer youth participants' interaction with the school as a space. In the focus group, Nich talked about how rumours and gossip would spread between fellow students about themselves and their sexuality such as: 
I remember one of my mates who I did date at one point, she's like my bestie but like she remembers hearing some school girls from the year group below us in the music room and they were like, 'Oh I think Nichola is bi-sexual', 'Oh my God. Really?' and then for any role we had in plays and stuff, I would play a guy and then, you know, for chicks who were like, 'If I was going to be like a little bit gay then I, maybe I, would go for the most masculine-looking girl so it would be like, we'll pick Nich'.

(Nich, 22, They/Them, Pansexual, Queer)

Many of the participants currently, or in the past, attend a single-sex secondary school. These participants said that their attendance came with the assumption that all students at that school were one gender.

Kate spoke about their experience of being in a single-sex school and shared with the group the following reflection:

... it was a single sex girls' school and it was kind of not necessarily the safest place to be a queer person, not because of violence or anything, just because of social opinions. It's like obvious that everyone there is fine, but also it's that anxiety about like people not wanting to talk with you, or people being uncomfortable with you, or like just like all these kind of thoughts that came up when you are fifteen and you know, just coming out and experiencing that kind of stuff for the first time.

\section{(Kate, 20, They/Them, Non-binary, Queer)}

In some instances shared to the group, there was the blatant lack of respect for gendervariant subjects in schooling spaces from the teachers. Compass shared their experience to the reaction of teachers referring to their chosen name instead of their ‘dead name’ (birth name) and said to me:

It wasn't even like, 'oh this is my preferred name can we use that?', it was mostly like the [school staff] would change it in the system but then teachers would just either forget and call me by my dead name or they would just be like 'I am not going to call you that'.

(Compass, 17, They/Them, Queer) 
Some participants also recalled moments where teachers would automatically assume students' gender and sexuality because they are enrolled at an all-girls school. In some instances, teachers made conscious decisions to not acknowledge some of the participants' gender expression and continued to use incorrect gender pronouns for them in class.

But on occasions where school staff made the effort to use Compass's new name, it made the space and experience a positive one. Compass said, “... actually my English teacher at the moment she is doing such a fantastic job, calling me Compass all the time and it's just like ‘aaaahhhh', and it just makes me happy”.

Friends were also able to make the school space more positive. Shay's photograph (Figure 25) shows one of Shay's friends in their university accommodation. Speaking to the photograph, they said, "A friendship. A friendship is a spark of light that has kept me so much more stable this past [university] trimester”. They too believed that friends made spaces feel safe and easy, and make up an important element to where Shay decides to spend their time. It was also important for Shay to see and interact with other people that had similar experiences and subjectivities to them in the spaces they engage with everyday.

Gendered school uniforms were discussed by some of the other participants in the focus group. In Compass's high school, there were a set male and female uniform. In sports teams and at school, Shay said they outright refused to wear skirts or dresses that were prescribed to them:

... when I was going into high school, the first thing I said in front of the principal was I am not wearing a skirt ... I just told them straight up I was not wearing a skirt and I never did.

\section{(Shay, 20, They/Them, Queer)}

If gender non-binary queer youth wanted to wear an androgynous uniform, or the uniform of their choosing, they would either have to formally apply or get parents or guardians' approval to do so. And for some of the youth who were not out to their family, this was not an option. There were also some more positive instances with regards to the students' uniforms. Charlie said that their old school is being a little more "forward thinking” and are changing to uniforms to either 'uniform A' (with a 
skirt) or 'uniform B' (with pants) and students were allowed to choose between the two.

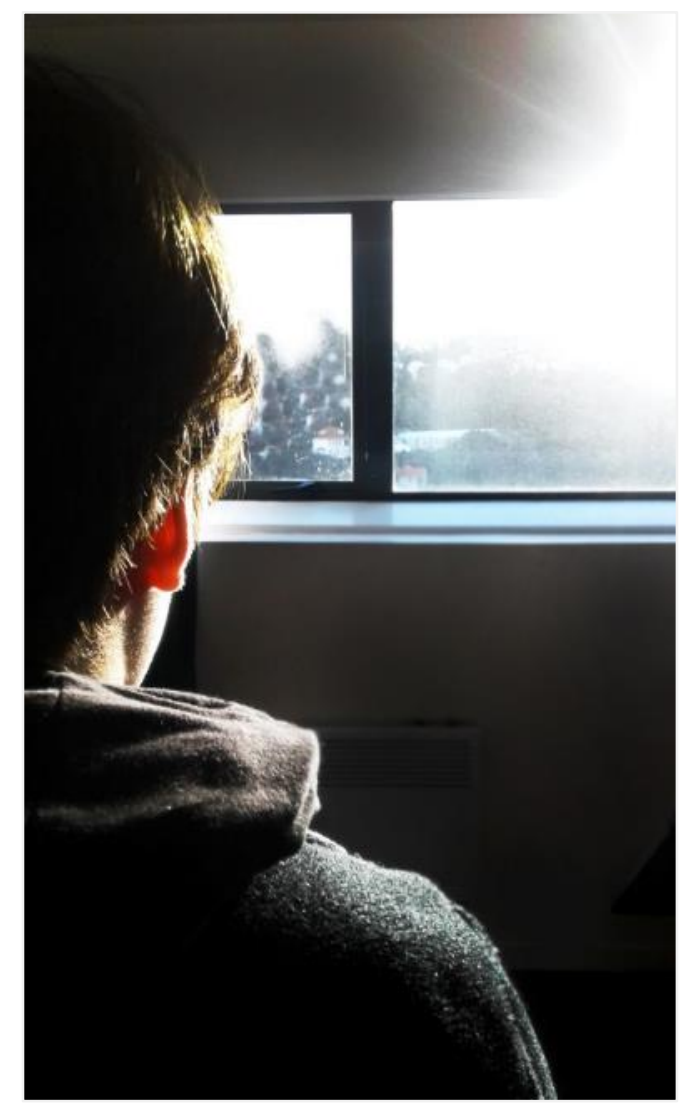

Figure 25 Friend is light, light is friend Yoda speak (Shay)

In regards to their sexuality, attending a gendered school as an out gay or lesbian student also had implications on how they were positioned in the school. Nich said that during their time at an all-girls school, they were routinely acknowledged as the “gay and queer kid”. In most cases this was not an issue for Nich, but because they were at an all-girls school, their subjectivity became a problem in spaces like bathrooms and changing rooms.

\subsubsection{Bathroom and Changing Rooms}

Queer youth spoke about their difficulty in engaging with bathrooms and changing rooms in the photovoice project. Although the inclusion of many gender-neutral bathrooms and changing facilities is becoming more common, there are still countless spaces where queer youth felt the need to 'pick a gender'. 
Kate took two photos of bathroom spaces (Figure 26 and Figure 27) where they felt displacement and rejection:

I am interacting with spaces that are gendered female, and I think I have a discomfort, no, I know I have a discomfort, no it is really changing my own language when I speak it aloud, like I know I have a discomfort with this, but I don't ever necessarily voice that discomfort or kind of, not make a fuss, or I guess even acknowledge it in myself that it bothers me. So I think it was good for this project to specifically look that in the face and be like, 'No, this does annoy me, this consistent binary and consistent needing to choose', and you know I truly believe [the] gender binary is [a] really harmful thing to people, especially young queer people who are still working themselves out, yeah.

(Kate, 20, They/Them, Non-binary, Queer - Kate’s emphasis)

Kate believed that they are pressured into the feeling that they "should" be able to fit in to one of these spaces because of the everyday, normalised presence of gendered toilets. These feelings manifest before Kate even enters the space. With only two doors offered to non-binary youth like Kate, often they are tasked to pick a door (or gender) that they are not. Kate said that being forced to choose what bathroom space to enter has implications and effects on what their "identity is and means" on a regular, everyday basis. To them, their subjectivity no longer exists in these spaces.

Compass also discussed being forced to pick a bathroom, but unlike Kate did not feel annihilated. Rather their choice depended on which binary gender category they felt that they most looked like on any given day. Through this contingent and flexible process, Compass eliminated the frequency of double takes, comments, and potential for violence in the bathroom, but Shay reflected on their experience of 'double takes' they received and the need for them to 'speak' before they might be accepted in the toilet of their choice:

I have always expressed myself this way since I was a little child. So child me, I was told go into the women's bathroom but then half the time I got told 'no, go into the men's bathroom' and then as I got a bit older I was getting double takes wherever I went. It just became an awkward situation because I've expressed myself in the same way. People that know this are fine, otherwise I get a double take until I speak.

(Shay, 20, They/Them, Queer) 


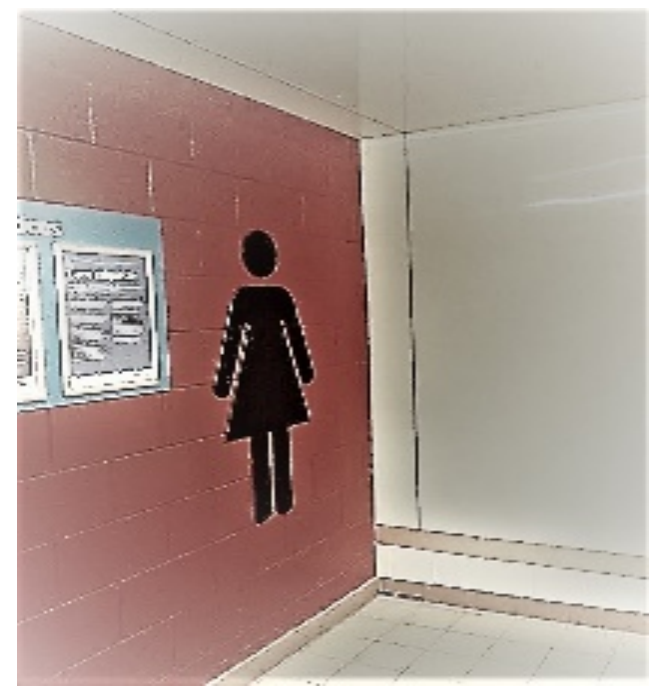

Figure 26 Gender Anxiety Part 1 (Kate)

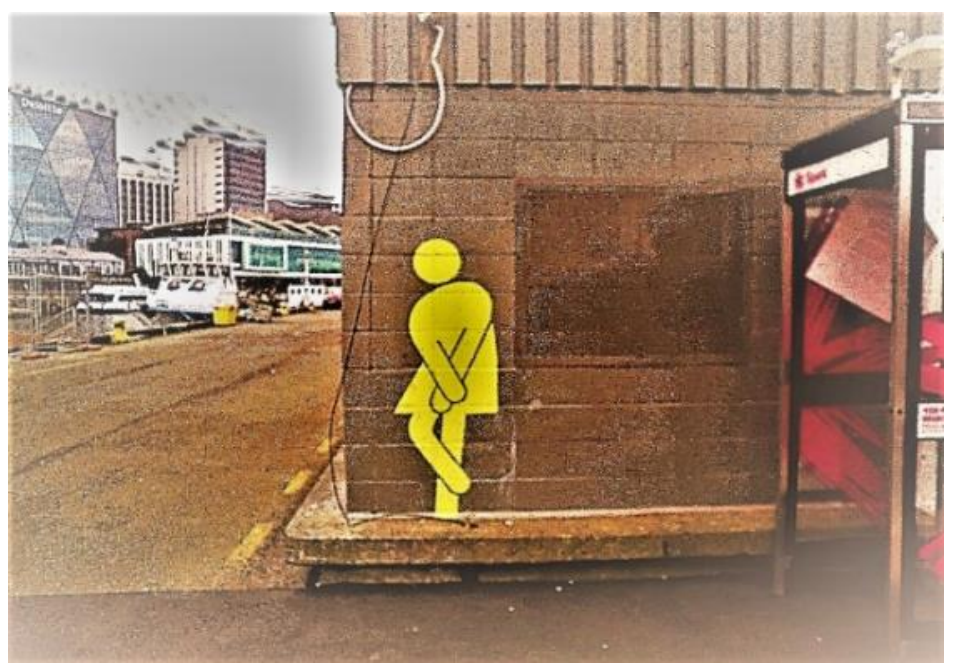

Figure 27 Gender Anxiety Part 2 (Kate)

Similar experiences also manifested themselves within changing room spaces (Figure 28). Kate shared an experience that was commonly felt amongst the other participants when they said:

... everyone else was like comfortable getting changed around each other. It was just something I could never do because I was sooooo freaked out that someone would think I was looking at them and like it was just such an intense fear that I hated PE so much.

(Kate, 20, They/Them, Non-binary, Queer) 


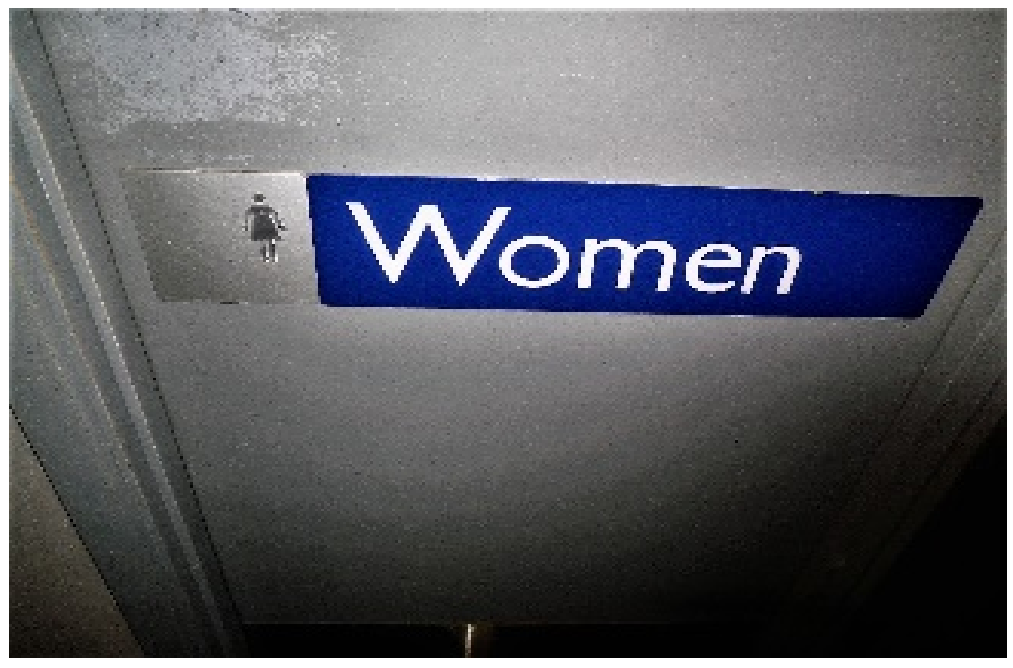

Figure 28 Gender Anxiety Part 3 (Kate)

Charlie also said that people at their school used to block them from entering the changing rooms because they thought they were lesbian and that they might "perv" on the other girls. Teachers would then punish Charlie for not being ready on time for gym class because they used to have to wait for everyone to leave the changing room before they could change.

These participants felt angry with these spaces and signage. Kate and Nich both found it difficult to be in spaces that were not consistent with their own understandings and expressions of themselves. The discomfort that all these participants felt was thought to be because of wider (and out-dated) societal assumptions that everyone falls into the male/female gender binary, something most commonly reinforced through iconography on bathroom, toilet, and changing room walls or doors. With regard to this, Kate said:

... she is wearing a dress, it is just like there is really obvious symbols of blatant sexism and misogyny and even this image [Figure 27] it kind of looks a bit awkward and bit funny, kind of like almost for me, especially it is making fun of women and it is kind of, doesn't sit good with me and I guess for my own identity it is kind of like, 'Oh you are ...'. Everyone is assuming that 'cause you are in that bathroom, [it] means this and this and this about you.

(Kate, 20, They/Them, Non-binary, Queer) 
There were some contradictory dimensions of these spaces. Lillian shared a photograph with the group (Figure 29) that showed one way queer people can reclaim spaces like the bathroom and changing room and challenge binaries through performative acts.

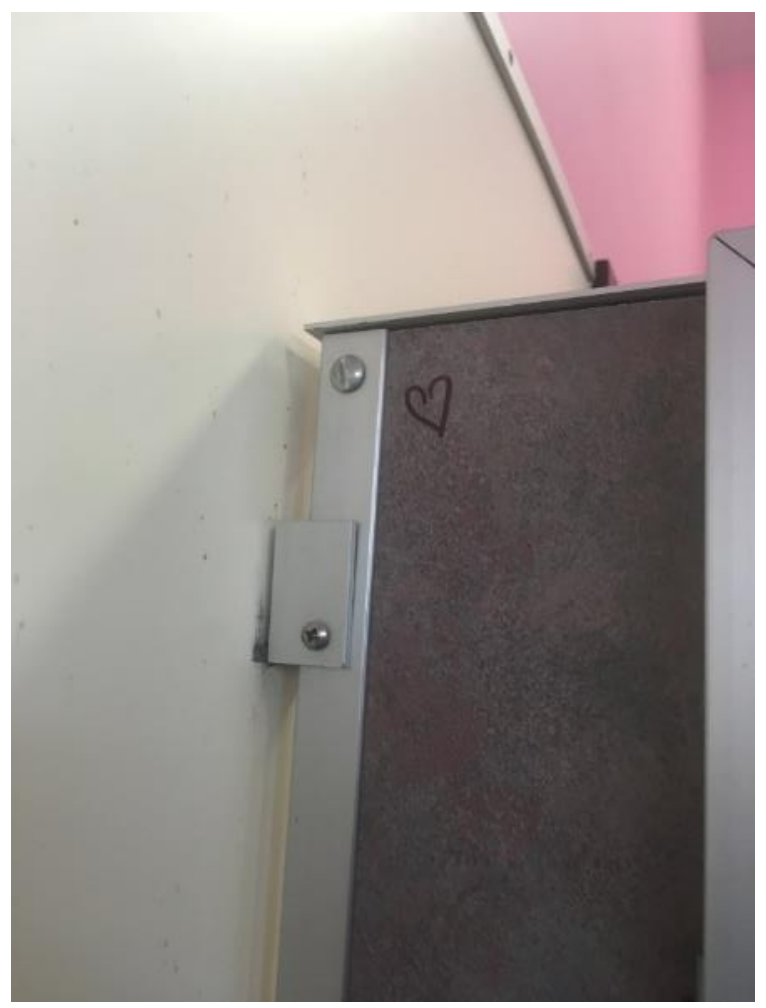

Figure 29 Bathroom Stall $<3$ (Lillian)

Lillian's photograph is taken from inside one of the toilet stalls at their school which shows a small heart drawn in permanent marker on one of the cubical panels. Speaking to the photograph, Lillian said:

... the stuff that gets graffitied on bathrooms at school is always like super personal or inspirational so I feel like it's always messages like, it’s cheesy stuff like, 'Be who you are, I love you', but it's at the same time, it's anonymous and very personal which I feel like a lot of queer kids can relate to, like finding communities on the internet and stuff, and revealing themselves to total strangers but also not connecting with the people like right next to them. So I feel like that is kind of reflected in the way that people leave these like 
anonymous but very very personal but heartfelt messages like in the bathroom stalls and stuff.

\section{(Lillian, 16, She/Her, Female, Queer)}

The bathroom wall, in Lillian's eyes, is a space that can provide anonymity to youth that want to express their innermost thoughts. It becomes a message board where reflections and thoughts can be heard by others, but are often never answered. Physical connections between people are made, but never in person. Designed to separate people in the stalls, it does not separate the thoughts and connections of people and their thoughts.

\subsubsection{Sports Teams}

In the photographs taken by the queer youth, sports teams and sporting spaces were sites that were heavily enforced along gendered binaries.

Compass shared their story about being forced to move from the male gymnastics team to the female one once the coach realised that they were female assigned at birth (FAB). The coach only realising this when Compass wore a 'feminine' Winnie the Pooh t-shirt to class one day and questioned their gender. Compass said that they saw a very distinct difference between the teaching in the male and female teams, mentioning that the males were doing more 'masculine' things like hand-stands and cartwheels, while the female team were doing more "basic" moves like rolly-pollies.

Even more of a negative experience for Compass was the language used by the coach in this very experience. Compass spoke openly to the group telling us that the coach spoke of them in a way that removed any form of subject or personhood, saying; “... [the coach] took me over [to the females team] half way through a class and they were like 'this is in your girls' group now'” (emphasis by Compass).

Instances like this express how heteronormativity is engrained in everyday spaces like that of sporting spaces - and the effect it can have on queer youth in them. Compass's subjectivity as a queer, gender non-binary sportsperson is precariously located spatially on the edge of both the female and male teams, with neither one accepting Compass in. Rather, Compass is seen as a 'this' not a person. Gatekeeping mentalities that control entry into 'male' and 'female' teams were often described to me as moments that required the queer youth to pick a gender to be included. 
Gendered stereotypes were also discussed on two occasions during the project. Compass said that when they were younger they enjoyed to climb trees and because of this would often get called a tom-boy. And again in a sporting space, Nich shared an experience at their local karate dojo:

I have super hairy legs, well not super, I mean they are quite hairy, but yeah I remember I was doing some stretches one day and then this woman was like really nice, and she looked at me and was like 'That's disgusting!' at my hairy legs ... I guess she wasn't used to body hair on young girls.

(Nich, 22, They/Them, Pansexual, Queer)

\subsubsection{The Stage}

Artistic spaces like the theatre and performance stages, and the expressions like drawing, acting, and poetry that were performed in these spaces, provided positive experiences for all of the participants that engaged with such spaces everyday.

Shay took their photograph of the theatre stage (Figure 30) at their university just before an audition. Shay explained that one of their biggest passions is to be on stage and to do creative things. The experience of the stage was clearly a positive one for Shay, as shown by our discussion in the following excerpt:

Matt: what is it about performing that you enjoy?

Shay: being able to be something authentic and to share it with people and I guess in a sense be something that I wish existed when I needed it

$[\ldots]$

Shay: ... I think it's the mixture of the people I am around, and the fact that on the stage for performers is a sacred space and when we go onto there you are passionate, you focus on the craft and if you're performing to people you are in serious mode. It, I don't know, is a sacred space and it feels pressuring but safe in the sense that if I am passionate about my craft, it will be okay.

(Shay, 20, They/Them, Queer) 


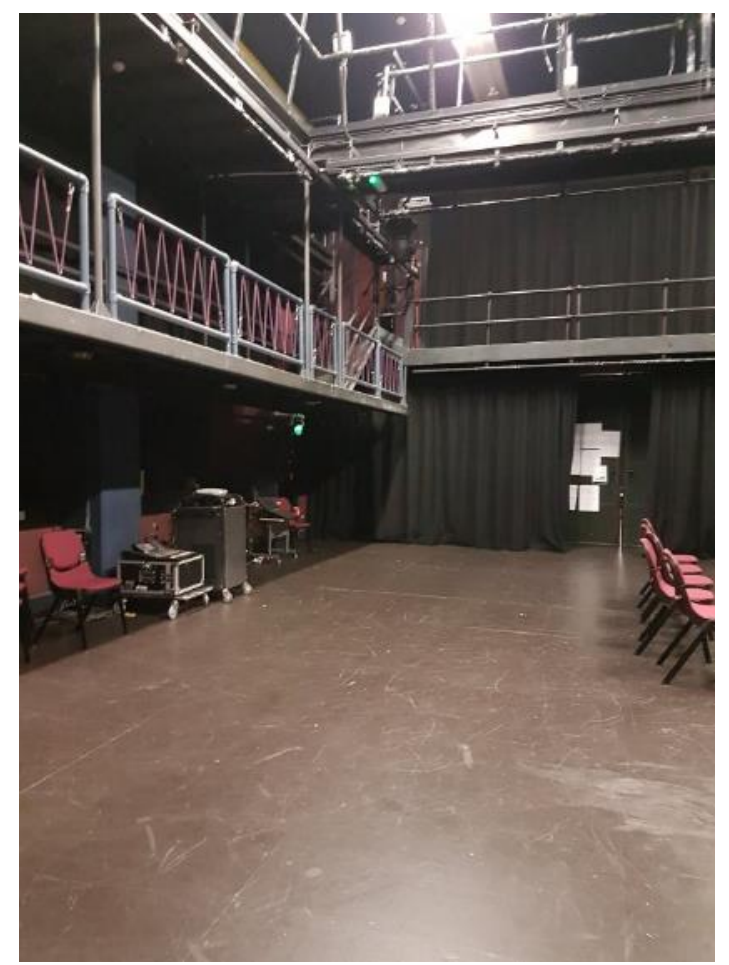

Figure 30 The Stage is sacred, so are you (Shay)

Kate had very similar experiences to Shay when performing their poetry on stage. For Kate, the ability to get up on stage, have control of the dialogue, and to share their views and experiences was often not afforded in other spaces they frequented. Kate's experiences, portrayed in their photographs (Figure 31 and Figure 32), were shared in their interview:

I think in these spaces, I am allowing myself to break down all of the constructor's stuff and allowing myself to engage in a conversation and dialogues that sometime I choose to not engage, whether it is for safety, or like, convenience, cause sometimes it's like I don't want to have a conversation with someone about gender or sexuality because it is like quite taxing and I do it a lot for a job, so just doing it in my free time is like, arrrrrg. Yeah, so it was kind of nice to have these spaces where I was being asked to speak and like I was having my voice and opinion valued and people were listening regardless of where they came from and what their experience of that kind of stuff was.

(Kate, 20, They/Them, Non-binary, Queer) 
Kate said that in these moments they were able to share facets of their queer subjectivities, about relationships they have had, and mental health challenges - all parts of their subjectivity that they never really discuss in other public spaces. They said that they feel like they are breaking or contrasting the everyday narrative of gender and sexuality in these spaces.

... in these spaces I am being given permission to change that narrative or, just present my own, whatever it may be.

... asking people to speak and share because it is totally on them on what they present and it is not, you know, you have to be this, you have to be that, it is like, no - tell us who you are, rather than us guessing.

\section{(Kate, 20, They/Them, Non-binary, Queer)}

But there is an air of caution in these spaces, something Kate says creates an "on the edge” feeling in these spaces, because they are sometimes unaware of the people they are performing to and may have to tailor performances to the audience and their views. Compass portrayed what Kate called an 'on the edge' performing space in one of their photographs too. Compass enjoys playing a wide variety of musical instruments, and piano is their favourite. But when their piano teacher invited them to play at a local church, as shown in Figure 33, Compass said they felt uncomfortable about the situation. According to Compass:

I always hated performing and it definitely comes back to that thing of 'I am my own person'. This certain thing [playing] doesn't need to be for anyone else, this is for me, and then going to those recitals was always like you have to bare your soul for these people that don't like the way you look, don't like the way you play, and it was kind of like, I didn't like it.

(Compass, 17, They/Them, Queer) 


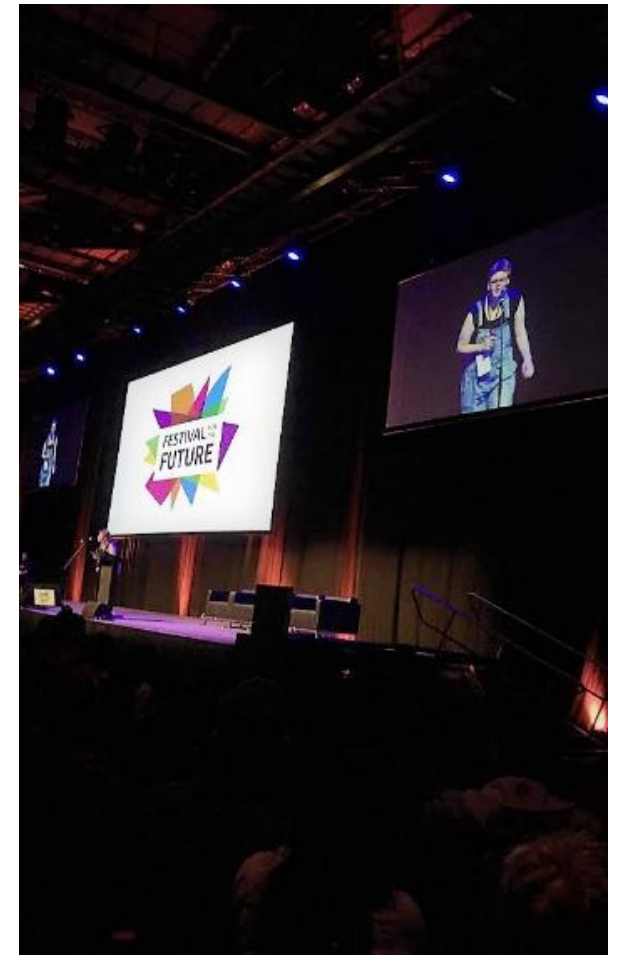

Figure 31 Honestly Performing (Kate)

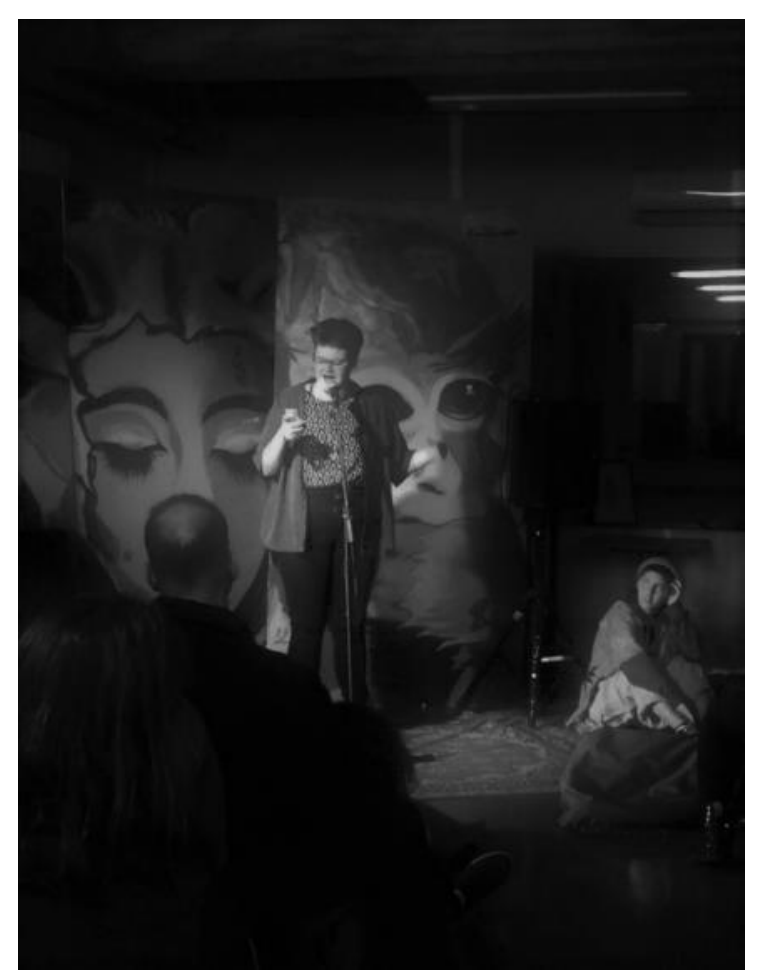

Figure 32 Performing Honestly (Kate)
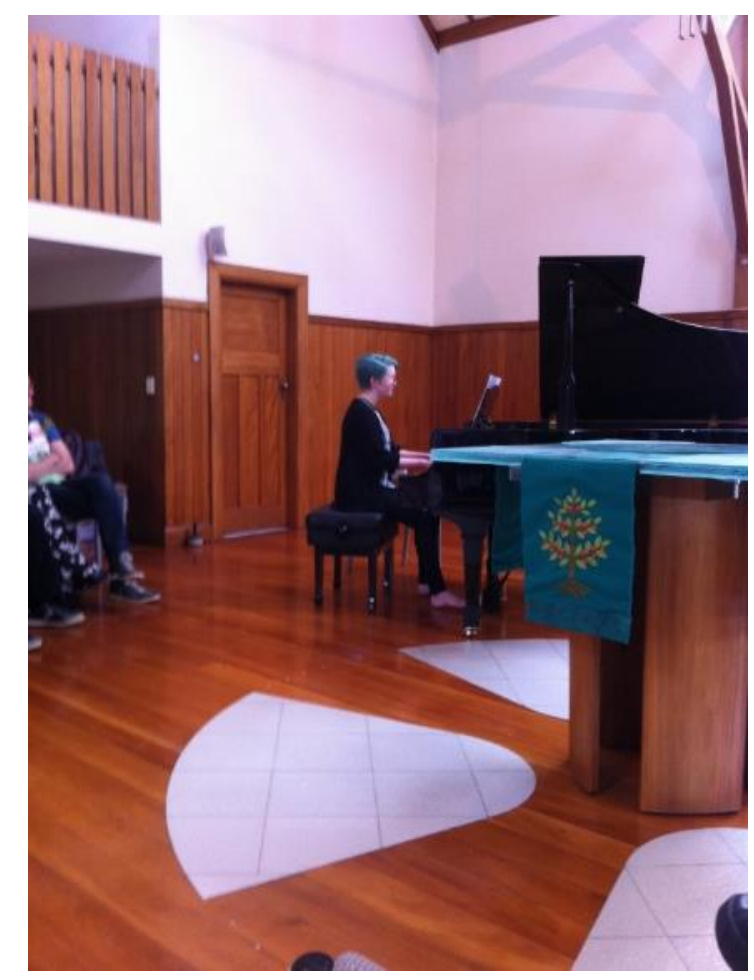

Figure 33 Church was never safe for me (Compass)

For Compass, the act of performing to the audience at the church was difficult. Fear arose because of the pressure to perform by their piano teacher in an environment that they already knew would have differing opinions on their subjectivity. Compass 
believed that instead of focusing on the music they were to perform, the audience would be more focused on what Compass was wearing. They detailed their emotions of the photograph and said:

I was standing and there was these crowds of families with their little kids and I was standing there and I had turquoise hair. I was wearing these finger-less gloves with skulls on them. I was wearing black and not wearing any shoes. I was like, 'I look so different' and I can feel that everybody just wants me to stop looking different ... What you can't see, but everybody there was so white, so suburban, so normal, and then I had my turquoise hair.

\section{(Compass, 17, They/Them, Queer)}

Compass said that they prefer to play in spaces where they are not being judged on appearances, but rather on the art form that they are performing. For them it is important that they first feel like they can be safe as a creator and as a performer before their art can become loved and appreciated. One of these spaces that allowed this to happen for Compass was on the stage in their drama group growing up. It was an example of how flexible and safe performing arts spaces can be in respect to performers' gender and sexuality while also speaking back to the normal and heteronormative constructions of subjectivity:

... in my drama group it was kind of you got the role that you were best for, not based on genders, so like we have a lot of like FAB people playing male characters and vice versa and it was kind of like you got the role that you were good for. It was definitely a lot of younger children or teenagers so it was nice to see that.

\section{(Compass, 17, They/Them, Queer)}

Much like the other spaces discussed in the photovoice project, the queer youth believed that the people in the space - the artists, performers, and creative people on the stage - contributed greatly to their positive feelings of the space. The queer youth believed that these people are a lot more open and aware to the world around them, in particular because of their need to portray and embody other characters, life worlds, and experiences on stage. In front of these people, Shay felt like they could fail but yet feel okay about it. There were not many spaces Shay felt like this was possible. 


\subsubsection{Church}

Like in a previous photograph, religious spaces were photographed by Compass to represent the dominant presence it had at one point in their life. Their experiences were symbolised in Figure 34 where the composition of the photograph helps to portray the feelings of a dominant and restricting pressure that they felt in religious spaces. When we discussed the photograph, Compass said:

The people in the church were very nice, I was friends with them and all that, but it was definitely like that thing of like I was the 'other' and I would like walk into wherever we were having a service and I would be immediately be like 'there is nobody else here that looks like me, at all'.

... I feel like Protestant churches are just like a lot more high and mighty in ways that they just like, 'I understand the real world but I also understand religion so I am better than you and I can tell you. So yeah it was definitely quite overwhelming to be around but also being like I don’t think I have ever believed in God but I kind of had to.

(Compass, 17, They/Them, Queer)

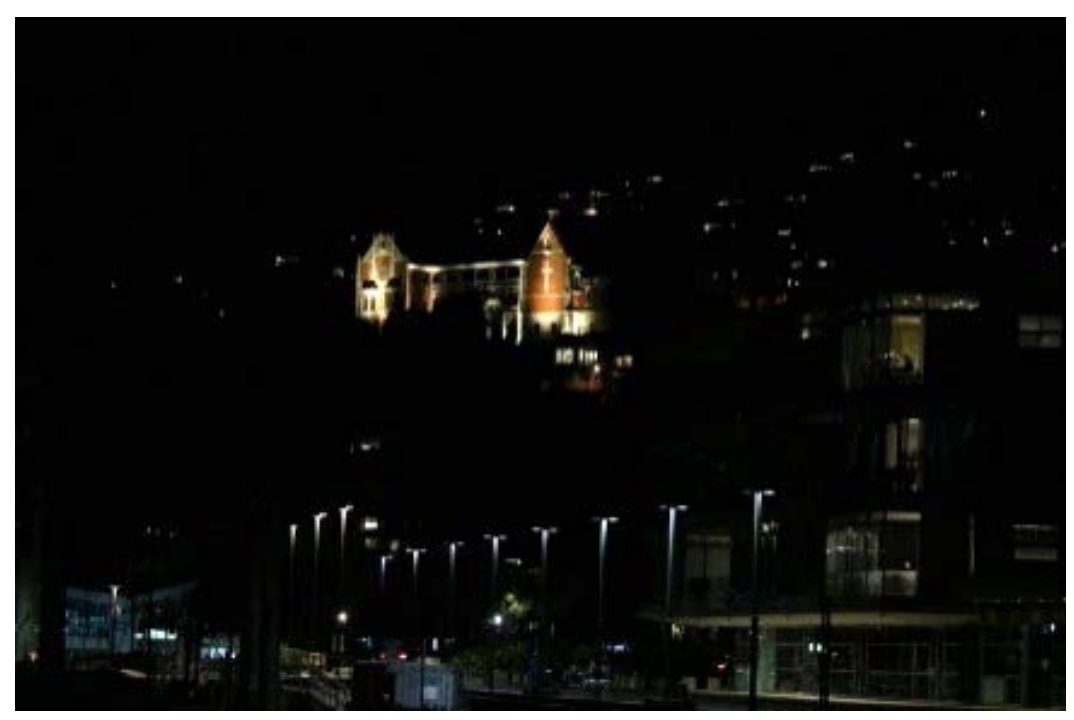

Figure 34 Church was never safe for me Part 2 (Compass)

Compass's experiences were also dominated by the feeling that they knew there was not really a space for them to bring up different ways of thinking and believing in the 
church. Being queer in religious spaces was seen by Compass as something historically negative. The space became particularly difficult to navigate when Compass's family required them to attend church together. Compass further explained “... it was definitely kind of trying to figure out 'If I leave this church will I have a family afterwards? What are the repercussions of leaving here?’”.

Compass said that they felt very fortunate growing up. Compass's parents would allow them to dress however they liked. One on occasion, Compass shared a story about their parents allowing them to attend church in fancy dress:

My mum was really good at letting me just cut my hair, dress how I wanted, she was more focused on the fact that we felt comfortable which I was just like '\#blessed', so I don’t think I ever, I can’t recall ever being like I am going to stop dressing this way but I do remember, and again at church, it was the first meeting we had the first time we went to the church that my parents still go to when we moved here from England, I dressed up as a Padawan, as a Jedi, cause I love love Star Wars and I just remember feeling so self-conscious.

\section{(Compass, 17, They/Them, Queer)}

However, Compass reflected on this experience and said that even though they got the opportunity from their parents to dress how they wanted, they got push-back from the church. The church's power on their subjectivity was felt by Compass, saying that the church felt alienating and lonely. They saw the church as a space of uniform and identical people, all prescribed by the faith and the teachings of the church.

Although Compass felt the pressure to conform to the normal look at church, they told me proudly, "I am never going to wear something that I felt uncomfortable in". Compass said that this feeling of being true to themselves comes from their mother.

I think my mum always drilled into me that, or I might just be like how I think is, that being your own unique individual is really good. So it didn't really bother me that I didn't look like other people in the church, it mostly bothered me that everybody else thought that I should look like them.

(Compass, 17, They/Them, Queer) 


\subsubsection{Marae}

When culture and space intersect, Shay found it difficult to negotiate their subjectivity in these spaces. These were especially prominent where traditional cultural views on gendered bodies contrasted against their 'gender expression'.

Shay detailed their experiences as being Māori in different spaces, like the marae (meeting house), and said that:

I mean I am Māori and all the time it's very very gendered, men stand at the front, the women stand at the back, there's tapu [spiritual and social code] regarding men and women and how they interact, everything you do and say, and makes me really confused and uncomfortable cause it's kind of like the public toilet thing again, only its more cultural and when family is integrated into that (sighs).

(Shay, 20, They/Them, Queer)

When present in such spaces, Shay said that they feel the need to "hide, hide, shut down, avoid, low-key try and fight and then walk off or something”.

\subsubsection{Online}

The engagement with social media, online, and internet spaces were discussed in the project with varying experiences.

For Kate, who uses online spaces like Instagram and blogs to speak about their experiences as a queer person, said that online spaces are a positive thing:

I think the online space informs a lot of what I do, 'cause like it informs a lot of what all of us do at the moment, yeah, I think it's quite helpful. I like it because it means I can interact with my friends, family, and people I kind of know and don't really know all at the same time.

\section{(Kate, 20, They/Them, Non-binary, Queer)}

Kate also shared their thoughts about how online spaces can form 'communities' of queer people. They said:

I think I feel more safe online because I know that all my queer friends are going to see stuff, whereas in public it is just me and like, there is like the whole power in numbers thing and power in community and being connected 
and especially on Facebook like I can have conversations with people who are in Auckland and what's happening up there.

\section{(Kate, 20, They/Them, Non-binary, Queer)}

Although a positive and safe space for the queer youth, Kate did mention that they have to filter friend and follow requests to their social media accounts to make sure the space is maintained as a safe one.

Charlie also thought similarly to Kate, and said that the Internet enables queer people to find people "who are like us" more easily. It was also a space to share inside jokes, express feelings, and receive support. For Charlie and Nich, the ability to grow up with access to the internet (and the myriad of resources the internet holds) made a positive difference in developing their queer subjectivity. For instance, Charlie told me about their feelings on the Internet by saying:

... when I was first exploring my identity and sexuality it was nice because the internet is there, it's like a bigger dictionary than the dictionary, because printed stuff becomes out of date so quickly these days and the internet changes (snaps figures) as quickly as we change so you can keep up to date with everything. So it was nice learning all these new terms and seeing how they fit with me, especially when I learned about pansexual. When I first started learning about sexuality and stuff I was like there's gay, lesbian, bisexual and this was back when it was like 'bisexual means you're attracted to boys and girls, not two genders or more' and then I found out about pansexual and I was like 'oh yeah, it feels comfortable, it fits'.

\section{(Charlie, 22, They/Them, Non-binary, Pansexual, Queer)}

Nich also shared their experience and told me that “... in high school I just remember being on tumblr.com and somebody mentioned non-binary stuff and so I read up on it and I was like 'oh this kind of feels comfortable”.

\subsubsection{Activism Spaces}

All of the queer youth felt that queer groups, such as the QSA sessions, InsideOUT meetings, and after-school queer activities were spaces that provided them with feelings of safety and positivity. These space are especially designed for the inclusion of queer youth, provide opportunities to express their queer subjectivity, learn from other people, and socialise with people of similar experiences. They were also spaces 
of opportunity to create change and increase awareness to the struggles (and pride) within the queer community.

One of these groups which was attended by Lillian was 'Schools Out', an after school group for queer youth. Lillian told me that:

Going to Schools Out was proabably my first time being in a queer space, like in my life, or at least in my adolescent life, yeah I look back on those memories so so fondly and even now I know that like going up there I definitely will be in a safe space.

\section{(Lillian, 16, She/Her, Female, Queer)}

Kate's photograph of the entrance to the InsideOUT office (Figure 35), brought up the way the spaces can give queer youth the space to be themselves. Kate said that when in a queer group space:

I can be mentally ill, I can have anxiety, I can be queer, I can have contrasting opinions and thoughts, I can be unsure of myself, I can fail, all these beautiful things in this space .... I think [queer people] are a lot more open about having conversations about other things specially for me like mental illness and I think that kind of I guess intersectionality and stuff about honouring the Treaty and like we understand, most of us, have an understanding of why this stuff is important cause we felt that ourselves, yeah.

\section{(Kate, 20, They/Them, Non-binary, Queer)}

There were also photographs of activism being used by participants to reclaim and queer different spaces. Compass shared a photograph (Figure 36) with the group of a sticker on a stairwell wall at their school. The 'stand together' sticker was from an anti-queer bullying event organised by Compass. Speaking to the photograph, Compass explained to me why they are engaged in promoting queer subjectivities and safety:

I would say that I like doing that kind of stuff. I like being proactive ... I like making sure that people are educated and know and that. I know how it feels when you see something that kinds of validates you, so I was like, 'I am going to do that for an entire school of people'.

(Compass, 17, They/Them, Queer) 


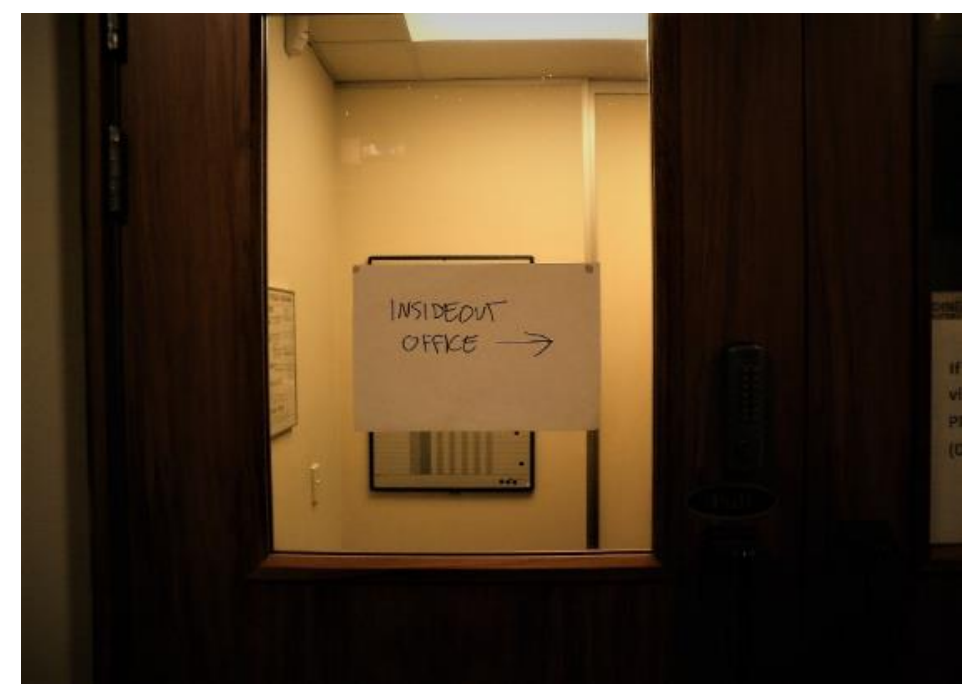

Figure 35 Coming Home (Kate)

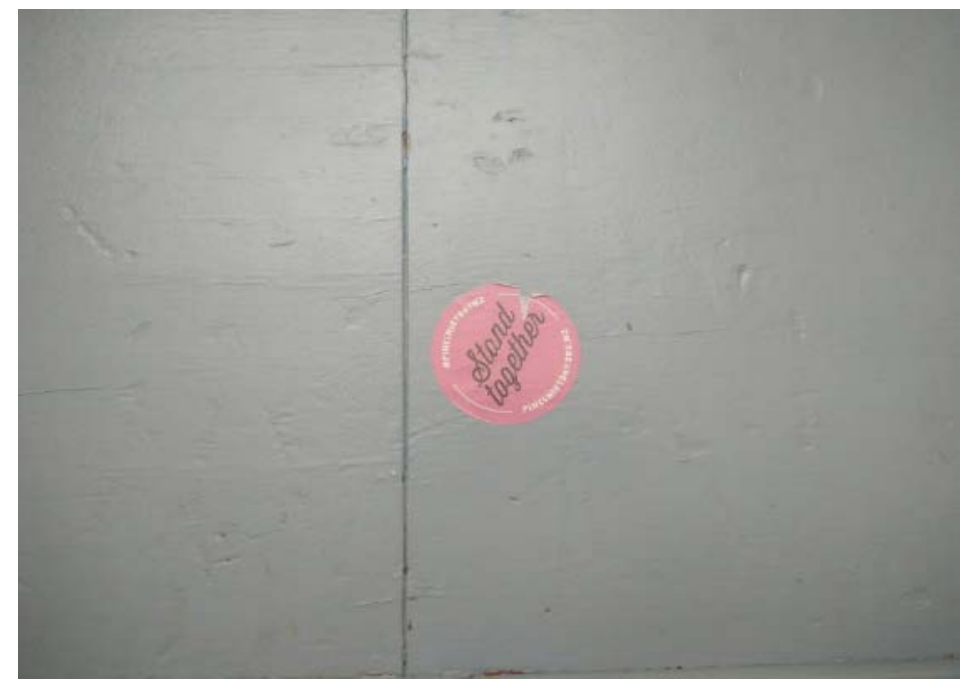

Figure 36 Stand Together (Compass)

\subsection{Private Spaces}

Finally, the last section of this chapter groups photographs of spaces deemed to be private by the queer youth. These spaces align with historic views of the public/private binary around the right to privacy to express sexuality and gender in one's home, but also highlight how heteronormativity is still present within certain private spaces and under particular circumstances for queer youth. 


\subsubsection{Home}

Queer youths' subjectivity within the home was highly dependent on how parents viewed ideals around gender and sexuality. As already portrayed in cultural and religious spaces, these values are shaped by other beliefs and discursive structures that impact on how youth experience a space. This is no different in the home.

One of the most common discussions on the experience of the home was the way language and culture were used in the home to signify or authenticate subjectivity, or not. Charlie explained that in the Chinese language there are no gender neutral words for girlfriend or boyfriend and husband or wife like there can often be in English (partner or spouse). This was also the case for Compass who said that in German, there are no gender neutral pronouns so it was impossible to refer to someone without assigning a gender to them. It made communicating about themselves and their lives difficult, either having to gender themselves or their partners.

In these home spaces, the inability to accurately and authentically describe themselves and their partners without gendering the language creates annoyance and frustration on the part of the individual. It also had implications on how subjectivity is validated within these cultural spaces. For Charlie, their subject position within their household and family spaces is confounded by the incapability to use language to explain their gender subjectivity or their relationship with Nich. It is portrayed by the participants that culture and language had real effects on how queer youth experience the home space and how they are positioned and validated in these spaces.

Charlie also spoke about their experience with culture in the home when they spoke about being both Chinese and queer in the photovoice project. When at home with their family, cultural norms around marriage, careers, child rearing, and home life are common conversations in the space. Charlie said, "I feel like with my mum and with my family I feel like there is certain expectations about how I should act”. Charlie's Mum still believes that one day Charlie will follow Chinese tradition and will marry a man, raise children, and build a career.

It was also expected of Charlie to conform to normalised gender roles within the house, even though they do not identify with a gender. Their mother expected them to do feminine type activities around the home - dishes, washing, and cooking - while their brother would be responsible for the more masculine chores like heavy lifting, or 
nothing at all. It was also expected of Charlie, especially as a younger queer person, that they should dress "more appropriately" for the gender their mother saw them as. Speaking of one occasion when Charlie began to dress more androgynously, Charlie said:

... my parents were like 'what the hell' and because my mum is incredibly feminine she like really pushes that onto my sister and I, she basically wanted us to be mini versions of her which is weird. Because you know we are our own people as well, but it was just really difficult and like it's only when I finally moved out that I could actually feel more free, even though I was doing it against their wishes, it felt like you were going against the grain.

\section{(Charlie, 22, They/Them, Non-binary, Pansexual, Queer)}

But, since Charlie was not meeting these expectations, they spoke about how they see their subjectivity within their family and said to me in their interview:

I'm the rainbow and the black sheep because a lot of things that I do my parents don't agree with - like they don't know about my tattoos and stuff. So I choose not to share a lot of myself with my family and that is a thing. And I originally wasn't going to come out to them but then that just kind of happened ... My Dad doesn't want to talk about my [queer subjectivity] which is also quite traditional ‘Asian man', where he’s like 'I'm just going to pretend this isn’t happening' kind of.

\section{(Charlie, 22, They/Them, Non-binary, Pansexual, Queer)}

Similarly for Compass, they also felt like they do not fit into their family because of their subjectivity and its place in their religious household:

I would say, my family, 10 out of 10, love them, but it was definitely like that thing of like I internalise everything and that I saw how the people in their church acted, or talked about [my queer subjectivity]. 'Oh that obviously means that [my family] think that as well'.

\section{(Compass, 17, They/Them, Queer)}

For these reasons, Compass found solace in escaping away from the house to another space in the back garden. When I asked what the feelings were that triggered Compass in taking the photograph of them on a swing in the garden (shown in Figure 37), they said: 
... when I lived [at home], it was kind of like that I felt like really closed off from my family and it was mostly because, like, queerness and they were really religious, I was like 'I am not sure how they are going to handle this you know', ... I would go sit at the bottom of the garden on that swing ... I use to draw there as well and that was kind of something I did, I looked at landscapes and I would sketch them.

\section{(Compass, 17, They/Them, Queer)}

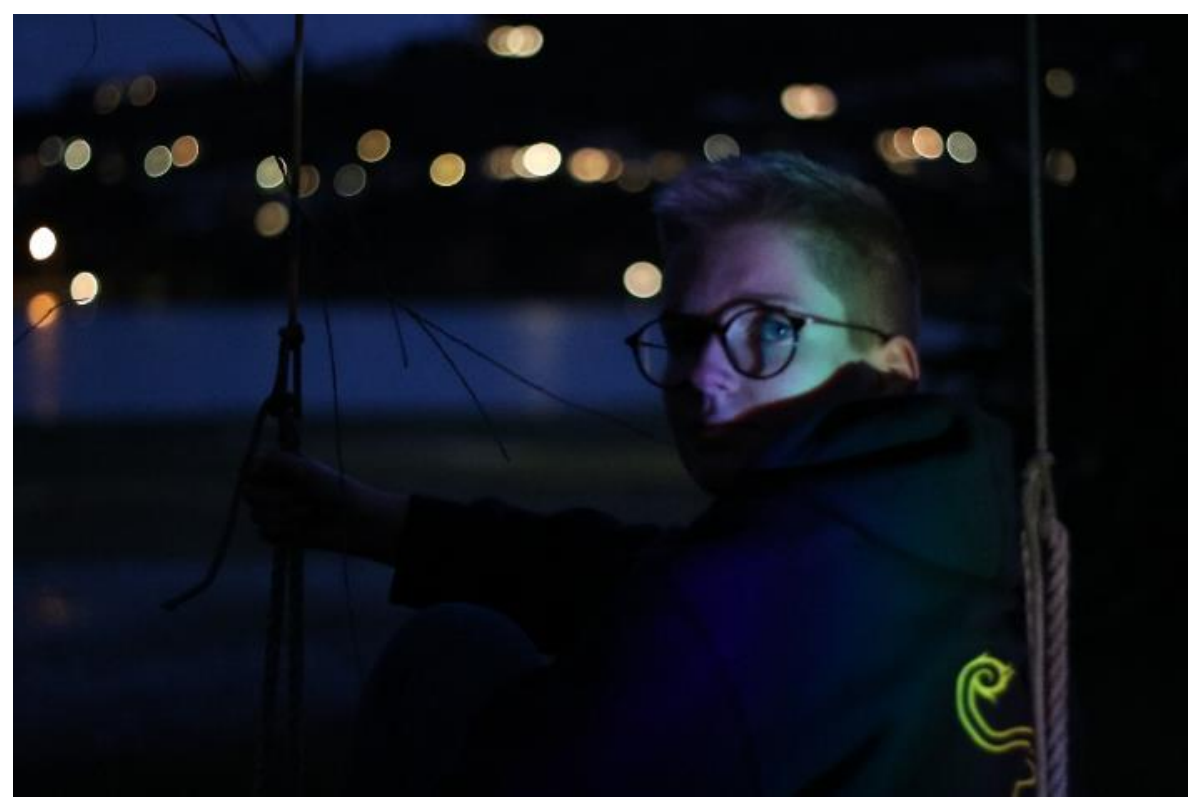

Figure 37 Cold, Serene, Safe (Compass)

In addition to the garden, bedrooms were also another space within the home that queer youth photographed for the photovoice project.

\subsubsection{Bedrooms}

Photographs of participants' bedrooms were frequent, with the bedroom providing security and privacy from the outside world, and a place to rest, relax, and be open about their true selves.

Nich and Charlie, who are partners, both shared pictures of their bed and bedrooms (Figure 38 and Figure 39). For Nich, their bed was an important space that they shared with Charlie. The ability to relax and be comfortable, eat, joke, sleep, and confide in each other were some of the shared feelings and experiences of bed spaces: 
The bed is where I hang out a lot with my partner. It's just us together being ourselves. Sleeping, eating, relaxing, and being romantic. I'm not sure I could be as open anywhere else, especially in public compared to this private space that's ours

(Nich, 22, They/Them, Pansexual, Queer)

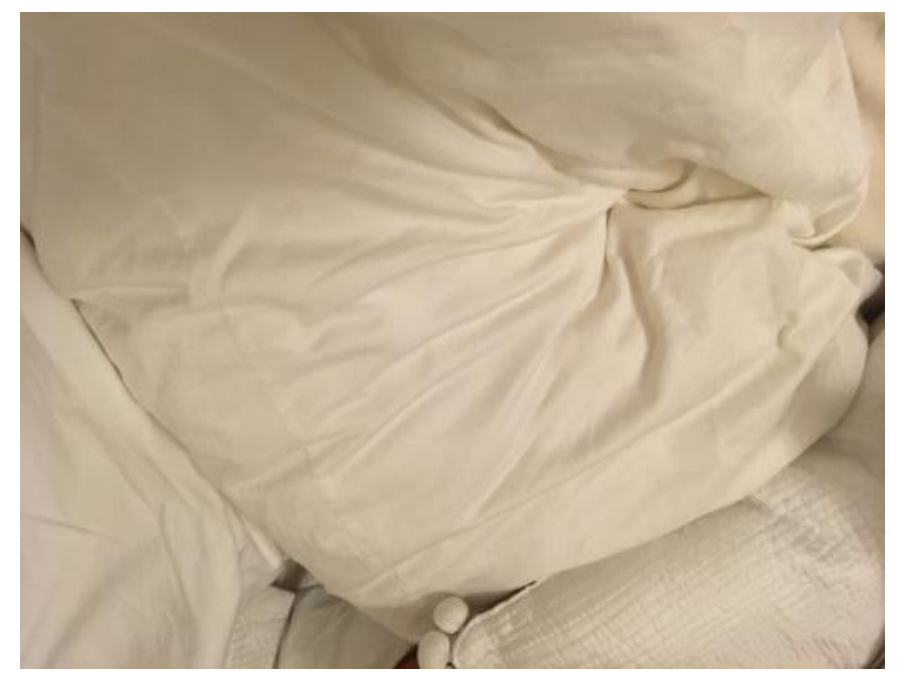

Figure 38 The bed is where I hang out a lot with my partner. It's just us together being ourselves. Sleeping, eating, relaxing and being romantic! I'm not sure I could be as open anywhere else, especially in public compared to this private space that's ours (Nich)

For Charlie, the bed was a space that had both physical representations of their relationship with Nich and memories of shared good times. It was a space they passed time, sheltered from the world, and where there was no expectations of 'normal' behaviour. Charlie said that they both liked to do art projects, like Nich's drawing and their crocheting in the space.

Some youth spoke about the ability to escape and separate from public pressure in their bedroom. Lillian said of her photograph of her bedroom (Figure 40):

So this is just the corner of my bedroom, just the safest space really, just to do whatever, to be whatever - nobody can see so we are fine, it's not in public. [In public] I kind of have to be aware, but [in bed] I can just do whatever, so just the ultimate comfort zone is the bedroom.

\section{(Lillian, 16, She/Her, Female, Queer)}




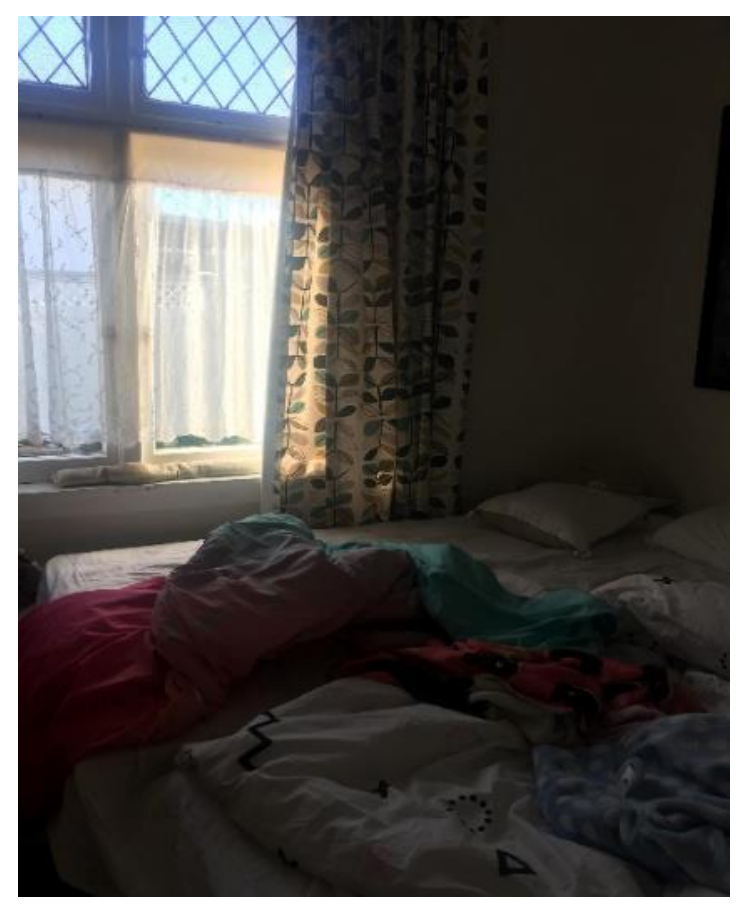

Figure 39 Comfort at its Best (Charlie)

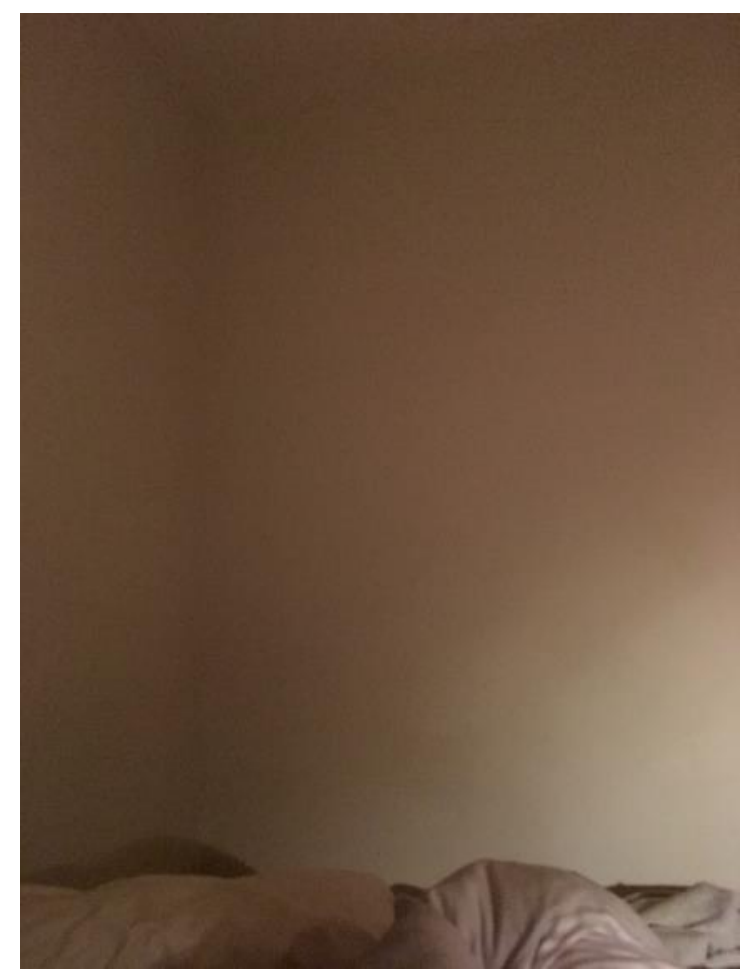

Figure 40 Safety of my Bed (Lillian)

And for Compass, the bedroom was a space to run to when times got tough:

I would say, I was home schooled as well, so I would spend a lot of time in my room, and just kind of, I would say I was quite closed off. 
I definitely think I am one of those people that my coping mechanism is 'instead of facing it head on I am just going to like close my bedroom door and not talk'.

\section{(Compass, 17, They/Them, Queer)}

But in a later reflection, Compass developed a different experience of their bedroom and said that the space became an important space that led them to coming out as queer. In the photograph seen in Figure 41, Compass began to discuss coming out and the meaning shown in the photograph:

... When we went to Germany, I stayed in my Mum's old room, and she had this wind chime that hung from one of the pillars in the room, and basically that room I think was the room where I discovered all of my queer identity. And the wind chime, the reason I did the wind chime instead of the room in its entirety is that because it had a wind chime in that room, I used to, for years, I use to wake up at midnight on the dot hearing a wind chime because I used to stay there so much and the wind chime would go during the night and I would just wake up and it kind of became a part of me so that room kind of is a part of my brain and part of my experience of figuring out who I was.

(Compass, 17, They/Them, Queer)

Compass explained to me that now when they hear a windspiel (wind-chime), they are transported back to that very bedroom and their memories of building their queer subjectivity by reading queer books, watching YouTube videos, and silently coming out to themselves.

In this same reflection, Compass shared two photographs (Figure 42 and Figure 43) that highlighted the growth they had achieved since they were younger while also using the set of photographs as a metaphor to speak to their younger self and reassure them that life in the future will work out. 


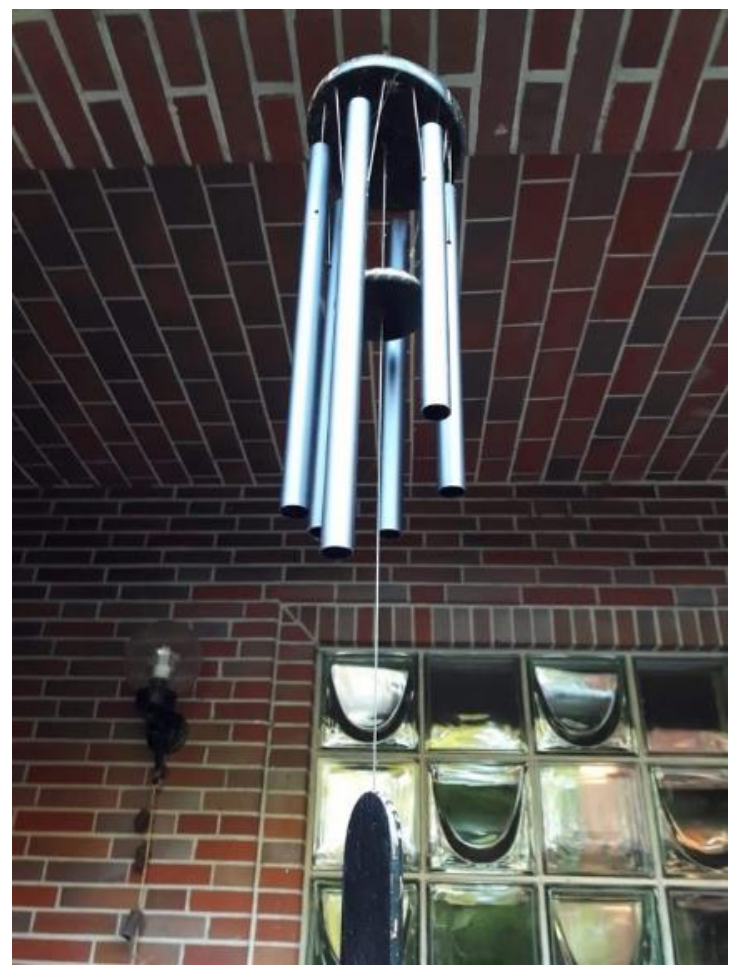

Figure 41 Windspiel (Compass)

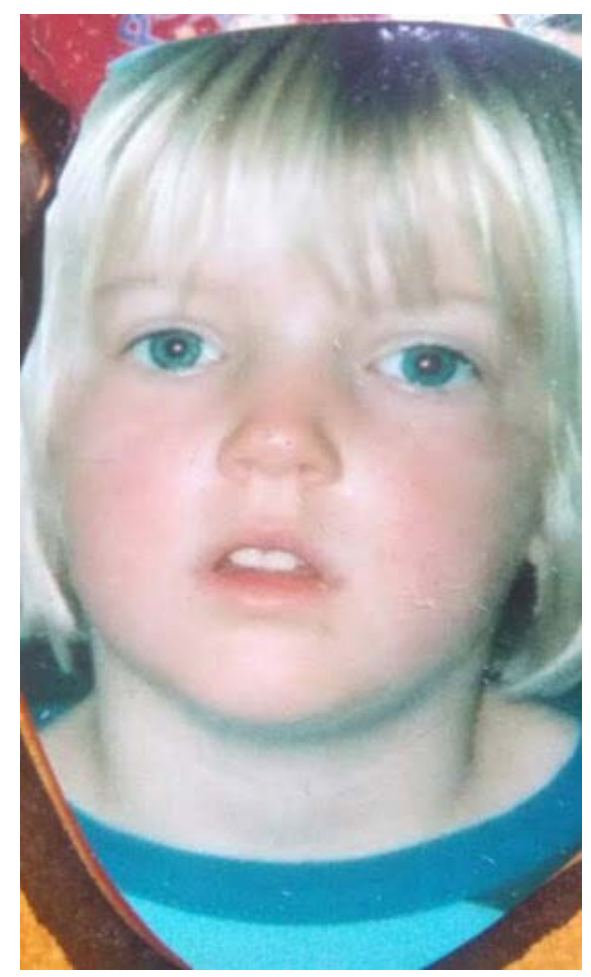

Figure 42 I'm grateful that I was allowed to dress up as Robin Hood as a kid because I know a lot of people weren't so lucky (Compass)

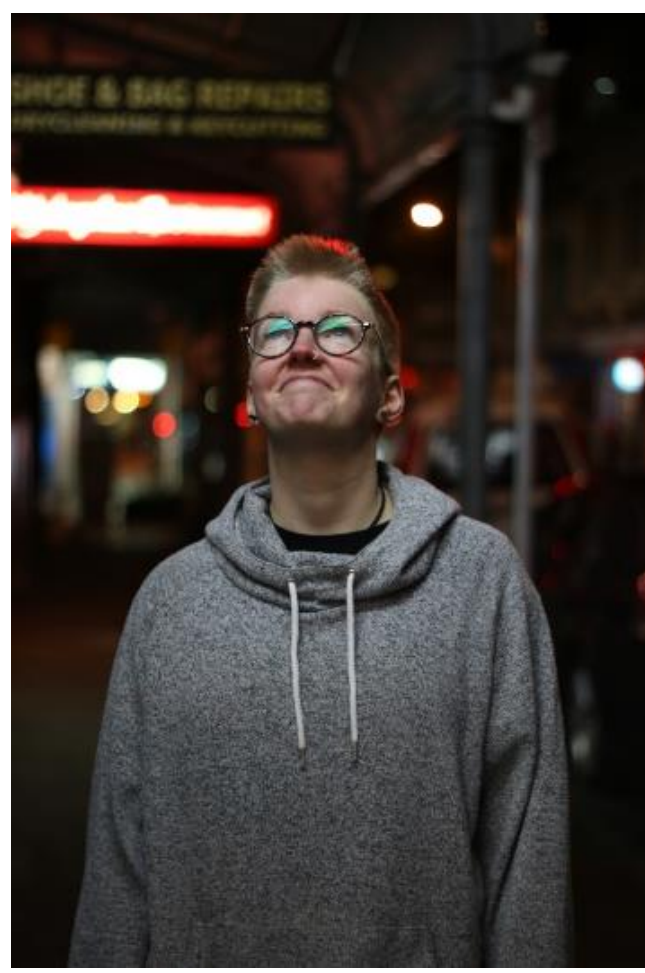

Figure 43 I'm happy to be myself (Compass) 


\subsection{Chapter Summary}

In this chapter, I presented the photographs and discussions of the queer youth participants generated during the photovoice project to work towards answering my first research question. These photographs - grouped into those pertaining to public, semi-public, and private spaces - show diverse understandings and experiences of queer youths' everyday spaces. As a result of heteronormativity, both positive and negative experiences of space were shown visually and discussed verbally calling attention to the effects of heteronormativity in their lives. Participants also identified spaces where they felt free from these discursive structures. Commonalities and differences between participants both in the photographs and during the focus group discussion were evident and will help to answer my remaining two research questions in my next and final chapter. 


\section{CHAPTER SIX: DISCUSSION}

In this chapter, I reflect upon and analyse the participants' photographs, interview transcripts, and focus group dialogue to better understand experiences of subjectivity, discursive structures, and everyday spaces. I begin by discussing how heteronormativity informed participants' experiences of public, semi-public, and private spaces. Following this, I detail what aspects of everyday spaces make them 'safe' for Wellington queer youth and how these aspects can be enhanced to counter some of the constraining or harmful effects of heteronormativity.

\subsection{Heteronormativity in Everyday Spaces}

For the queer youth in this research, heteronormativity - or the assumptions, practices, and beliefs that reinforce heterosexuality and cis-gendered bodies as normal and natural - was regularly portrayed as contributing to their experiences of everyday spaces. However, as previous work within geographies of sexuality have shown, the spatial and political dichotomy between private and public spaces can control, exclude, or accept different subjectivities (Duncan, 1996b), and influence the degree to which heteronormativity is imposed upon queer bodies.

\subsubsection{Public Spaces}

Regulation and control over gender and sexuality were most commonly experienced in public spaces by these queer youth. Within city streets and on public transport, queer youth felt the need to constantly be on the defensive, with many commenting on the feeling of isolation and exclusion from the space. Kate said it was very uncommon to get positive recognition within public space, saying that “... it is something that happens indoors and in conversations with people or online, I rarely feel affirmed in that kind of way in public”. Violence, threats, and negative comments also made public space uncomfortable and unsafe.

The cause of these experiences stemmed from how the queer youth were positioned as abnormal, or dissidents or transgressors of the taken-for-granted (heterosexual) norms of public spaces. Most commonly, this positioning took the form of policing of gender and sexuality through laughter, disapproving comments, threatening behaviour, or acts of violence from 'straight' others. Many participants experienced 
homophobic or transphobic comments from passing members of the public especially if these youth had shown visible displays of affection or were seen to be 'acting queer'. In other cases, heteronormative policing took on a less violent and confrontational form. Instead, participants were subjected to forms of what Foucault termed 'an inspecting gaze' as (straight) members of the public enacted disapproving stares. Participants were sensitive to these acts and internalised their implications which lead to self-disciplining behaviours in an attempt to conform to more dominant expectations of gendered and sexual subjectivities in these places.

Similar dynamics were at work in public spaces of transport. For example, Charlie, Nich, and Shay found buses and trains to be public spaces which did not convey a welcoming of individuality and uniqueness. They were aware that their oftenandrogynous fashion choices made them stand out from the 'darkness' and regularity of attire of others taking public transport to and from work (see Shay's photograph in Figure 15). They felt the monotony of commuting and the expectations of behaviour in these spaces made them different to the people around them and they were treated as such. People would often stare at them and make comments very similar to the ones experienced on city streets. Participants also noted the prevalence of heteronormativity through their attention to commonplace greetings using gendered welcomes like 'hello ma'am', and small-talk conversations involving the need to gender partners.

What became clear through this photovoice project was the connection between queer visibility and the severity and frequency of heteronormative enforcement and policing. As Shay and Lillian both experienced, if queer youth are able to 'pass' as being either heterosexual or cis-gendered within public spaces, the occurrence of threats, inspecting gazes, and the need to self-discipline declines. Being able to dress more feminine or masculine, being with friends of the opposite 'gender', and conforming to ritualised heteronormative behaviour enabled many youth to avoid being singled out in public space for being 'queer'.

\subsubsection{Semi-Public Spaces}

Ambivalence and variety of experience was most common in semi-public spaces, the most prominent of which was the stage. Even though the space is open to the public gaze from the audience, when queer youth were on the stage, they were able to transcend heteronormative understandings and expectations relating to gender and 
sexuality to some extent. Geographers looking into performing arts spaces have shown that music (Revill, 2000; Saldanha, 2005), drama (Nagar, 2002), and art spaces can be effective in giving power and control to people over the representation of their subjectivities (Rogers, 2012). The stage allowed queer youth like Kate and Shay to perform a subjectivity, character, or feeling how they wanted it to be portrayed, rather than one that was dictated to them by heteronormative expectations. The youth were given the authority to speak as a performer or artist and the right to be heard by an audience regardless of their sexuality or gender.

Participants' experience of nature presented it as space that youth go to escape away from the pressures of the world. In parks, landscapes, and on waterfronts, there no obvious or exclusionary feelings of heteronormativity which made them spaces where queer youth could just be themselves. Many of the participants put these feelings down to the fact that were often never many people in the space to be mindful of. It also was peaceful, tranquil, and contributed to positive feelings of one's self outside of their queer subjectivity also.

Online spaces were another semi-public space where participants were often able to be self-expressive and truthful about their subjectivities. Online queer communities used a number of strategies to limit heteronormative influences, like blocking friend requests, filtering through comments on social media, and making blogs open only to queer people and allies. But, they noted that other online spaces had the potential to alienate and exclude queer subjectivity through online trolls, cyber-bullying, and queer-phobic messaging.

Schools also highlighted the pervasive influence of heteronormativity. In same-sex schools, gender non-conforming participants were constantly faced with their abnormal subject positioning through the policing of uniforms and changing rooms. Gendered language was also a reminder to participants of their 'otherness' within this space. However, there were also instances where queer youth were able to create their own - sometimes temporary but private - spaces within the school: Regular QSA meetings became spaces that allowed for variation in gender and sexual expression and were a social space for students to meet. Student-led pride events, authorised by teachers, were beginning to resist and offer alternatives to heteronormative discourse 
by making clear that queer youth had a space within the school, even if it had to be 'closed' or censored to avoid potentially negative interruptions.

Marae were experienced as potentially exclusionary when queer youths' subjectivities conflicted with culturally-regulated understandings of normal sexuality and gender. Shay's experiences of being on their marae highlighted the significance that genderculture expectations have on someone that is both Māori and queer. Shay shared to the focus group that the tikanga (custom) that regulates these spaces makes for an uncomfortable experience at times. Heteronormative gendered roles (male/female) during a pōwhiri (welcome ceremony) or whaikōrero (speeches) dictate who can speak and where they stand on a marae. Māori who experience gender dysmorphia or see themselves as takatāpui (or queer) can often experience difficulty in their relationship with this space as a result, as Mika (2007, p. 139) noted:

Many takatāpui are allowed back on their marae ... as long as it is with an asexual visage. The thought of takatāpui taking a partner along to a function on the marae often causes visceral reactions ... Some takatāpui are prevented from speaking on the marae âtea [courtyard], even though tikanga dictates they can, due to some perceived equivocality over their gender.

In some regards, Māori tikanga can reflect forms of a 'heteronormative' discourse via its set of values of what are considered to be 'proper' kaupapa (practices) in relation to aspects of gender as McBreen, Waitaha, and Tahu (2012, p. 25) argue:

Just as in New Zealand's dominant European heteronormative culture, many of us who are not heterosexual have experienced silencing - we are expected to be discreet about our sexuality, while our heterosexual whanaunga [kin] are not. Many of us have been excluded at some time because of our sexuality, and some have been physically attacked. Too many have not survived. Tikanga Māori is often used as an excuse for this violence, hatred and fear.

However, they note that modern-day tikanga has become largely a heteronormative force because of colonial influence. The pre-colonial existence of sexual fluidity for Māori was documented and was accepted until Western medicine, the demonising of homosexuality in religion, and criminalisation under colonial laws were introduced (McBreen et al., 2012). 
Shay's subjectivity as both Māori and queer meant they fell within a nexus of intersecting subjectivities that both experience forms of spatial repression and control. 'Survival' might mean choosing between their sexual subjectivity, their Māori subjectivity, or living two separate lives - one as a 'straight, cis-gendered' Māori or one as a white ‘queer’ (McBreen et al., 2012).

Religion was also another factor that enforced heteronormativity and influenced experiences of semi-public and private spaces. Religious enforcement, Taylor (2016, p. 80) noted, is not just experienced within the place of worship, it "infiltrates the everyday, intimate, and political spaces of family, community, and subjectivity, which are shaped and stretched by gendered sexual-religious relations”. Heteronormativity is often promoted as 'natural law' in a range of religions, and gendered and sexual binaries are policed through theological or spiritual teachings and practices. For queer youth who are spiritual or have a religious faith, such beliefs and practices can result in their marginalisation. For example, Compass felt like they were treated as an outsider within the church and the home. Luckily however, their parents accepted Compass and their choice to stop attending Sunday Mass, but for others, the consequences may be significantly different. Internal struggles with personal acceptance of one's queer subjectivity combined with the external conflict with close family and friends can create additional pressure on queer youth in spaces where they ideally would be able to go for solace and support.

Public toilets and bathroom spaces also revealed challenges and contradictions. Lynda Johnston (2019) believes that the bathroom space is created for, protected by, and favours heterosexual and cis-gendered persons. She sees two reasons for this: The first comes from notions of dirt, impurity, and how and where 'proper' bodies go to get clean:

The contemporary toilet is a place where gender variance and homosexuality are linked to dirt, disease and public danger. Those who are recognizably trans are subject to persecution for using the 'wrong bathroom' in ways that are not only callous and cruel but compulsive and curious. The urgency with which one seeks to clarify the gender identity of another or to expunge gender-variant folk from the public lavatory entirely is beset by worries about disease and disorder that, in the present day, are overlaid by angst about radicalized and class-specific purity (Cavanagh, 2010, as quoted in Johnston, 2019, p. 63) 
Secondly, it is commonly assumed that the need to 'protect' vulnerable heterosexual women from predatory heterosexual men justifies the existence of cis-gender-specific spaces like bathrooms. Without this division, media purport, some men may dress or appear as women to enter into a female space for threatening reasons.

Hence, bathrooms and toilets are places where “... all bodies ... become objects of other people's gazes so that our gender is under scrutiny every time [queer people] use a bathroom” (Johnston, 2019, p. 68). In Johnston’s thinking, Kate, Charlie, and Nich’s experiences presented here could be said to be subject to Foucault's ideas of the inspecting gaze, self-correction, and self-surveillance. When a body is deemed to be 'out of place' - like when gender-variant or trans people enter into a gendered bathroom - queer people adopt strategies to blend in and become inconspicuous.

For these queer youth, the everyday battle of engaging with gendered bathrooms led to both an unpleasing experience of space, and also the marginalisation of their subjectivities. In having to perform to the regulated gender of the bathroom, the queer youth spoke about their feelings of discomfort and loss of subjectivity. Subject positions begin to erode away in these spaces to the point where their very existence is viewed as dirty and improper. Bathroom spaces epitomise heteronormative spatial discourse and continue to be a site of debate as activists work to assure better rights and access for gender variant, trans, and gender non-binary persons.

\subsubsection{Private Spaces}

Within private spaces, queer youth were able to find solace within the space knowing that there were no heteronormative expectations on their behaviour, like the ones that were experienced in everyday public and semi-public spaces. Participants saw private spaces as a location for self-discovery and subjectivity development. It was here that they came to understand their feelings about sexuality and gender without fear of violence, retaliation, or regulation.

For these reasons, the bedroom for many was a sanctuary, where participants chose to go for privacy, romance, reflection, and to simply be themselves. Bedrooms provided a retreat from the heteronormative practices present in their wider private spaces like their family homes (Schroeder, 2015). When queer youth were unable to perform to normalised subjectivities and meet the expectations around traditional marriage, high 
paying careers, child rearing, and gendered roles within the home, they often felt isolated, excluded, and viewed as an 'other'.

For Charlie, these expectations of appropriate behaviour and subjectivity were further compounded by cultural expectations on gender and sexuality. Although Charlie is out to their family, the continual heteronormative expectations from their mother that they will grow up to be a "good Chinese daughter" placed Charlie within what Švab \& Kuhar (2014) call a 'transparent closet' within the home. The transparent closet refers “... to those social situations where a child's homosexual orientation is acknowledged within the family but is not further discussed. Parents (or other family members) refuse to accept and deal with the consequences and meanings of the new information” (Švab \& Kuhar, 2014, p. 20). The lack of acknowledgement and acceptance of Charlie's subjectivity by their mother resulted in them feeling like the "rainbow sheep" within the family. Charlie mentioned that their mother would still refer to Charlie and Nich (their partner) as 'friends' to close family and friends to avoid the discussion on having a gender non-binary and queer child.

Language used within the home was also another way in which heterosexuality and cis-gendered subjectivities were normalised. For two participants, the absence of identification terminology in their native languages impacted on their experience of private spaces. Charlie spoke about the lack of gender-neutral terms for words like 'partner' in the Chinese language, which made it incredibly difficult to explain their relationship with Nich. Similarly, Compass shared that in German, they cannot use gender-neutral pronouns or terminology to explain themselves or to show relations to other people. The participants' inability to accurately and authentically describe themselves and their partners through language created significant implications for how queer subjectivity was positioned and experienced within the home.

\subsubsection{Conclusion}

In conclusion, within most public spaces, there are clear and distinct heteronormative regulations of the interactions between space and subjectivity which queer youth experience as mostly negative, but within semi-public spaces, queer youth are also able to transcend beliefs of normalised sexuality and gender that construct these spaces and begin to rework them by carving out more inclusive, 'private spaces' within them. 
Private spaces vary too, with some being subject to very strict public regulation - like toilets - and others being freer, such as bedrooms.

The participants' discussions and photographs also reflected that private spaces are heterogeneous and cannot be assumed to be 'safe' in contrast to 'unsafe' public spaces. For some queer youth, they do enjoy the freedom of sexual and gender expression within their private spaces. Bedrooms become sanctuaries for individual expression and relaxation. However, if or when parents and family enforce expectations on how queer youth should perform their gender and sexuality, private spaces can feel rather open to public enforcement. Culture, religion, and language in the home become tools through which heteronormativity is experienced and regulated.

Ultimately, it is evident that these queer youth navigate heteronormativity every day in almost all spaces. Gender and sexuality are policed by the general public and regulated by threats of violence, homophobic/transphobic comments, and through inspecting gazes. Language, culture, and religion further conflate the experiences of heteronormativity and lead to feelings of isolation, excision, and othering. They also impact on how some queer youth view and manage different aspects of their subjectivities within a particular space like a church, marae, or family home.

\subsection{Safe Spaces and Feelings of 'Safety'}

As noted in the previous section, safe (often semi-public or private) spaces were vital in creating positive experiences in queer youths' lives. However, because of varying subjectivities, daily routines, and access to safe spaces, there were different views on what a safe space was and what it meant to feel safe. Considering the everyday interactions queer youth have with heteronormativity and its subsequent effects on their experience of space, it is important to understand where queer youth find safety and how this can be enhanced. To do this, I present and analyse the spaces participants viewed as 'safe'. Much like the work of Rosenfeld \& Noterman (2014), my focus in this section is to not create a 'recipe' for queer safe space, rather to “... treat safe space as a living concept, identifying tendencies and variations in its use, and recognizing its situatedness in multiple contexts” (p. 1347).

\subsubsection{Safe Spaces and What Makes them Safe}

Four common spaces were identified to be 'safe' for queer youth. The most photographed of these were in natural spaces in which queer youth could go to escape 
away from the world since there were no pressures to conform to heteronormative ideals or no need to censor their subjectivity in these spaces. The participants believed that nature offered a form of anonymity as many felt their subjectivity did not matter or define them when engaged in such spaces.

Performing arts, music, and art spaces facilitated the ability to be self-expressive, take command, and provide a platform to speak about what may not be heard in other spaces. Artistic spaces were also a space to make new friends and build relationships with like-minded people.

Online spaces gave a voice to many of the participants and provided them the right to discuss their experiences and be heard by accepting people. Here they could discover new understandings of sexuality and gender, reach out for help, and support others.

Finally queer groups like the QSA sessions and InsideOUT meetings and offices were spaces that provided all participants with safety through opportunities to express their queer subjectivity, learn from other people, and socialise with people with similar life experiences.

Within these spaces, there were three common feelings of 'safety' that were expressed:

For participants, the opportunity to be authentic and honest about their mental health, passions, and relationships as well as being able to display their subjectivities through affection and dressing androgynously were important. The ability to share all aspects of one's subjectivity in a space without fear of retaliation reflected Hartal et al.'s (2014) understandings about the importance of being honest, sharing opinions, and having the opportunity to reveal one's 'true' self.

Secondly, safety was being able to escape from heteronormative pressures and enabled participants to be free from the weight of being a sexual and gender minority for a period of time. Lastly, having other queer people around provided feelings of safety and the ability to share experiences, thoughts, and time. Comradery, relationships, and friendships generated some of the strongest feelings of safety in everyday spaces.

In the rest of this section, I explore these dimensions further with reference to participants' visual and verbal texts and existing published research. I first discuss in more detail aspects of spaces that contributed to participants' feelings of safety. Next, 
I detail how youth queer heteronormative space as a method of constructing safety and reflect on the role that conforming strategies had in the creation of safe(r) space. Lastly, I present the challenges that queer youth face in constructing safe space.

\subsubsection{Aspects of Safe Spaces}

Photographs of safe spaces showed two aspects of Hartal's (2017) elements of queer safe space (as noted in Chapter 3.2.3). InsideOUT offices and QSA meetings were spaces of inclusivity, where the agreed upon ways to communicate and accept each other created the right to dignity and respect while removing the fear of violence, stigma, and shame experienced elsewhere. Nature and bedroom spaces created a sense of anonymity contributing to individuals' right to privacy. In these spaces agency rested with the youth to either disclose their subjectivity, or not. These spaces contrasted with bathrooms and changing rooms where the right to anonymity is often removed, and queer youth must disclose their subjectivity in order to enter the space.

However, these were the only two elements of Hartal's (2017) queer safe space that were apparent. This may come down to the fact that Hartal's view on the aspects of safe space centred on human rights logic and creates a utopian view of safe spaces for queer people. It may also be that the model reflects adult priorities rather than those of young people.

Within my research, the absence of explicitly enforced or regulated heteronormative gender and sexuality binaries, evidence of queer representation, and the presence of friends or animals were also contributors to the construction of safe spaces.

In spaces where rules and regulations on gender, sexuality, and bodies were removed or contested, participants found safety and comfort in being able to 'be themselves'. As this discussion has already shown, the stage, the bedroom, and online were all spaces that did not enforce dominant discourses. Within the semi-public spaces, queer youth were also able to build spaces of safety for themselves and other queer youth at times, or find ways to resist heteronormative expectations.

These visible and physical representations of queer subjectivity in everyday spaces provided safety in the feeling that the queer youths were 'part of the everyday', appreciated, and respected. Safety developed in these spaces because queer youth perceived the space to be accepting of their subjectivity and this increased their confidence to express themselves and/or resist heteronormative discourses. 
The presence of people, friends, or animals in a space also contributed greatly to feelings of safety because they acted as a bridge between safe and unsafe space. The presence of friends and animals often put the participants into their own micro-space, secluding them from the rest of the world and transforming feelings of danger, discomfort, or neutrality into safety. The introduction of people and animals changed the composition and configuration of previously space and the bodily relations within it (Rosenfeld \& Noterman, 2014).

\subsubsection{Young People Conforming to and Queering Space}

Reflecting similar strategies noted by Gill Valentine (1993), queer youth in this research were able to use conforming strategies to minimise the visibility of their queer subjectivity and create more feelings of safety for themselves as a result. For example, in bathroom and changing room spaces, some talked about sheltering in the corner of the space and "mind[ing] their own business" when getting dressed to minimise risks of being seen as a voyeur. Others youth did not enter school changing rooms until everybody had left to avoid confrontation with the other students.

In public spaces like public transport, positioning of their body to face away from other passengers helped Charlie to resist the mundane, routine, and heteronormative feeling they encountered, and elsewhere Shay decided to avoid their marae in order to evade the conflict of having to decide whether to stand with males or females in the space.

Participants were also able to draw on the internal spaces of memories and their imagination to stay safe in external spaces. Some spoke about the fictional stories and romances they would tell students at school to fit in with the normalised sexuality expected there. While some took to altering their appearance and creating a fictional 'straight appearance' to fit in with parental expectations and conform to normalised family life and to be less noticeable in public spaces. In moments with animals (Figure 12 and Figure 13) and through reminiscing about 'coming out of the closet' in an old bedroom space (Figure 41), participants also created distance from negative experiences of the spaces around them, reducing anxiety and enhancing feelings of safety.

These examples are clear signs of Fox and Ore's (2010) argument of queer people's inability to construct, and fully experience, safe space. Instead they feel safe(r) through the use of negotiating and conforming strategies to limit possible aggression and 
violence. In doing so, they were able to create more feelings of safety for themselves but also served to reinforce heteronormative discourse within these everyday spaces. Rather than taking a stand against heteronormative discourse, conforming strategies alter, remove, and diminish queer subjectivities to meet the expectations heterosexual and cis-gendered spaces have. Kate said that their experience of conforming to the norms of bathroom spaces had serious implications and effects on how they viewed their own subjectivity.

However, I stop short from concluding that it is never possible to construct safe space. Through the use of queering space strategies, queer youth were able to make their place in the heteronormative world and stake claim to their own queer spaces. The most prominent way was through activism. Through overt actions like pride events, drag shows, placing queer-affirming stickers around school, and online blogging the youth participated in strategies to undermine heteronormative control. Such strategies aimed to push queer-friendly messaging and stigmatise phobias against queer subjectivities and personhood.

Activism has been at the heart of queering's focus, beginning with Stonewall in 1969, and although the youth saw their actions as comparatively small, Kate believed that, “... its small things that change cultural consciousness and cultural narrative”. Small actions towards the queering of space were also important on city streets - generally understood to be unsafe public spaces. Seeing representations of queer subjectivity, discussing queer things while in the street, or by being with a queer person actively changed the power relations in the space and provided comfort and safety.

Queer youth also used digital spaces to subvert heteronormative discourse much like Brown (2000) and Sedgwick's (2008) ideas of 'closeted' space. Brown (2000) saw the closet as a space where queer people often perform their gendered and sexualised subjectivities. In the 'closet' queer people are either able to conform to or transgress heteronormativity. Either way, such spaces are central to queer people's performativity and construction of their queer subjectivity.

Acting like the closet, online spaces provide a location where queer bodies can begin to work against the heteronormative forces present and this, “... allows queer people to form and understand their queerness and represents a space where they learn how to do queer” (Fraser, 2010, p. 32). For the queer youth in this research, online spaces 
helped them work against oppressive heteronormative forces (through actions detailed by the participants like coming out on social media or anonymously on blogs), understand what queer subjectivities might mean (through searching for the right terminology to use for their feelings), and interact and connect with other queer youth (through chat rooms, Facebook and Instagram pages, and blogs) (Fraser, 2010).

It is vitally important for queer youth to have access to online spaces, as Fraser (2010, p. 31) continues to mention, because the:

... [online] space is highly necessary in contemporary society because people are exploring and identifying their sexuality at younger ages, thus rendering public spaces (e.g. pubs, bars, and clubs) traditionally used by queer people inaccessible for individuals at stages where they are most in need of support and community.

\subsubsection{Challenges in the Construction of Queer Safe Space}

The strategies discussed above do provide some safety to queer youth in their everyday spaces, and can queer space for periods of time, but many challenges still exist. Firstly, the lack of safe spaces identified by the participants in the home and in 'private' highlight the challenge of queer youth building safe spaces within close proximity to parents and inside the familial home. Hartal (2017) believes that a safe space should incorporate the right to control one's self and the space one is in, however, with queer youth this is not always possible. The influence and control from parents, whānau, and caregivers can limit queer youths' access to safe spaces, especially within the private sphere. To develop access to safe spaces within the home, queer youth need to have more accepting and open adults in their private spaces (Sibley, 1995).

Secondly, the feelings of safety often do not feature in space with definitive boundaries. When one compares the experiences of queer youth to that of queer adults who have the agency (especially through access to capital) to create more noticeable safe spaces like queer saunas, nightclubs, and bars, (which are all exclusively created for, and by, queer adults), it is clear to see the how much more difficult it is for queer youth to create similar fixed and bounded safe spaces. Consequently, safety for queer youth is often experienced as temporary - like patting a dog or walking down the street with a friend. Perhaps not surprisingly, Knopp (2007) acknowledges queer people are likely to be "suspicious, fearful and unable to relate easily to a fixity and certainty 
inhering in most dominate ontologies of place” (p. 23). As a result, queer youth often find solace, comfort, and safety in being in motion, or nowhere at all (Knopp, 2007) and are used to making the most of transitional, temporary, and semi-anonymous spaces (Bech, 1997).

Thirdly, even apparent queer safe spaces may not always be inclusive of every queer youth. This research affirms Fox \& Ore's (2010) argument that it is impossible to claim homogeneous feelings of safety for queer youth especially within a diverse group of individuals and experiences of the everyday. During the focus group discussions, it was evident that some participants had contradicting opinions on what spaces were safe for them. Any explicit attempt to construct queer safe space therefore needs to incorporate and reflect other intersectional subjectivities to be truly representational of queer youth such as culture, ethnicity, religion, and body size/shape.

Lastly, it is vital to acknowledge that there can be exclusionary practices within the queer community that impact queer youths' sense of safe space. As Valentine \& Skelton (2003) noted in their research, marginalised groups can often create a safe and united space with borders of their own, valuing a specific subsection of queer subjectivity over others. This can be constructive but it is important to recognise that "[v]ulnerable social groups are not just marginalized/oppressed, but can also marginalize and oppress each other” (Valentine \& Skelton, 2003, p. 863). When this occurred on Cuba Street for Compass, for example, they felt isolated and further distinguished as an 'other' in their everyday space. It also undermined one of the key aspects of safe space for queer youth - inclusivity and sharing common subjectivities.

In light of these challenges, I now consider the possibilities for enhancing safe spaces and resisting heteronormativity in everyday space.

\subsubsection{Enhancing Queer Safe Spaces}

To work towards answering the remaining part of my last research question, I reflect on how queer safe space and its aspects can be enhanced to improve queer youths' safety in their everyday space.

To enhance safe space and increase access to safe spaces, it is important to acknowledge the immense role adults play in creating and defining queer youth and their safe spaces. Although queer youth need to construct their own safe spaces away 
from adult control, it is through adults' agency and access to capital that common safe spaces like the InsideOUT office, after-school groups, and QSA meetings are possible. When adult allies are located within family/whānau, safe spaces within the home and bedroom become more accessible too.

The role of activism is a positive aspect in queer youths' lives that contributes towards destabilising wider hegemonic structures in their everyday spaces. Positive experiences of pride events, sticker drops, and QSA groups highlight that activist spaces not only contributes to the destabilisation of heteronormative discourse, but also work to enhance other forms of acceptance and safety in regards to issues like mental and sexual health.

Queer friendly and inclusive images, representations, and signs on public streets are one small aspect that can be enhanced to increase feelings of inclusivity and safety. These messages should aim to incorporate other forms of subjectivity as well, including aspects like body size, culture, religion, and fashion sense.

Establishing and promoting safety to express one's sexual and gender subjectivities within cultural and religious spaces could help improve feelings of inclusivity in these heteronormative spaces. Queer friendly churches and marae, which have been set up in Wellington, are one clear example of this.

The participants were also very vocal about challenging the enforcement of gender performance within same-sex schools. If schools can take reasonable steps to remove the institutional enforcement of gendered performance within their school, and work towards addressing policing behaviour on the part of teachers and students, then one of the queer youth's most frequented spaces can become a lot safer.

\subsection{Chapter Summary}

My discussion chapter began with analysing and presenting the different ways that heteronormativity influences participants' experiences of their subjectivity in public, semi-public, and private spaces. As a result, this affected where and how participants also feel safety in their everyday spaces.

Public spaces were heavily regulated and controlled by discursive structures. Violence, threats, and inspecting gazes contributed to negative experience of city streets and public transport. Experiences of semi-public spaces were varied, with 
heteronormativity able to inform subjectivity in marae, churches, and school. More open and accepting spaces include that of nature, the stage, and online. Private spaces were also varied in their heteronormative enforcement, with bedrooms providing sanctuary to participants, homes reinforcing gendered expectations, and bathrooms becoming sites of gendered regulation.

Safe space for participants meant the ability to express one's self, being surrounded by friends and queer people, and seeing clear representations of their subjectivity in space. Safety was most commonly experienced on the stage, at QSA meetings, in bedrooms, and in nature. 


\section{CHAPTER SEVEN: CONCLUSION AND FUTURE RESEARCH}

My final chapter is dedicated to reflecting on my research journey. I begin by revisiting my research questions and distilling my key arguments and discussions. I conclude with reflecting on the limitations of my research and presenting areas for future research.

\subsection{Conclusions}

\subsubsection{Experience of Everyday Space and Subjectivity}

This research has been able to present meaningful photographs of, and conversations about, queer youths' everyday spaces because of the rapport built between the participants and myself, as well as because the photovoice method was able to create embodied and reflective data. Using visual methods in this research contributed to the ability of participants to reflectively discuss their experiences since photographs were able to, “... induce an awareness of place by holding up mirrors to our own experience; what had been felt can now be seen, what was formless and vacillating is now framed and still” (Tuan, 1979, p. 161).

Participants' photographs and discussions during the photovoice process show that their subjectivities played a significant part in where they went and the experiences they had in those spaces. As the focus of this research was to understand the experiences of the everyday and routine spaces of queer youth, photographs of school, transport, city streets, and bathrooms were frequent. Nature and the stage, although not an everyday space to all the participants, was an important space for queer youth to document for this project.

Most of the participants' photographs in this research were located within traditionally public and semi-public spaces, leading me to believe that queer youth experience their subjectivity the most in these such everyday spaces. I also consider the lack of photographs taken of private spaces, like within the home, may highlight that participants' subjectivity was not a contributing factor in how they experience these spaces. Or alternatively, that their experience of subjectivity was connected to more 
private aspects of familial spaces, like family, culture, religion, and animals, that were discussed more frequently.

For the most part, participants were content with how they experience everyday space. However, in specific spaces, heteronormativity did influence queer youths' experiences through exclusionary, unsafe discourse and practices which led to negative experience of their subjectivities in space.

\subsubsection{Heteronormativity in Everyday Space}

"It is through understanding the spaces with which one interacts that one can grasp the formative work of discourse on the body within space” (Fraser, 2010, p. 31).

In nearly all the participants' everyday spaces, heteronormativity informed how their subject position was placed. Many participants felt that their gender and sexuality were seen as abnormal or 'othered' in spaces because of society's ideals and norms around sexuality and gender.

Visual displays of non-heterosexual affection were met with negative remarks and physical appearance seen as polluting the space. Heteronormativity was also heavily enforced from 'straight' people through inspecting gazes, violence, stares, laughter, and passing in public spaces, while language, culture, and religion contributed to regulation of queer bodies within semi-public and private spaces.

As a result, bathrooms, public transport, and city streets were seen as unsafe and created the most common feelings of displacement and exclusion. Home and bedrooms were seen as both sanctuaries and as prisons for participants, and depended on parents, family, and whānau expectations on their child and acceptance for queer subjectivities.

In light of these experiences of heteronormativity however, queer youth were able to contend with negative discourse and experience safety in a wide range of everyday spaces.

\subsubsection{Queer Safe Space}

Experiences of safety in everyday space were varied in this research. Queer youth found safety when spaces enabled them to be true to themselves without fear of 
reprisal or danger. Safety was also being able to escape away from the pressure of the everyday and be surrounded by queer people and animals.

These experiences of safety were visible in spaces like QSA meetings, InsideOUT offices, nature, online spaces, and the stage. Bedrooms were also commonly seen as safe, with some exceptions. In these spaces, heteronormative expectations and norms were either non-existent or overshadowed and gave authority over to queer youth to create their own expectations and possibilities in the space.

Queer youth were also able to create their own safe spaces with their own narrative and expectations through queering space and conforming strategies. Activism abled many of the participants to work towards undermining the heteronormative discourse within their school and begin to create a culture of acceptance and pride within the space. Online spaces were also easily queered by creating, sharing, and regulating space for a queer only community that enabled participants to be honest with other accepting people. Conforming and performing strategies (Valentine, 1993) were important to overcoming risk within unsafe space, but came at the cost of freedom of expression and individuality while only creating instances of safe(r) spaces (Fox and Ore, 2010).

Challenges do remain for queer youth in constructing and accessing safe space. Intersecting subjectivities (like culture and religion) placed some queer youth differently than others in everyday space. There were also challenges from within the queer community in some cases about what is, or how people should be, 'queer'. Significant differences between the construction of safe space for queer adults and youth - like more transitional and temporary space and less safe spaces within the home - mean that age does have a noteworthy impact on both experiences of heteronormativity and access to safe space.

As a result of these and other conclusions in this research, I propose that Geography pay more attention to how safe spaces are experienced in different contexts. A solution that addresses all these concerns is one that shifts our thinking of what a safe space is and sees that:

... new [safe] places must be forged in coalitions through a process of struggle, examining our own assumptions and privileges, challenging not only others' ignorance, but our own ignorance, and seeking new ways of interacting with 
those who are differently positioned from ourselves (Fox and Ore, 2010, p. $630)$.

\subsubsection{Thesis Contribution: Transformative Action and Change}

Whilst this project was too small to create wide social change, it did conclude with three important outputs. First, as a key part of photovoice’s underpinnings (Wang \& Burris, 1994, 1997), participants benefited from taking part, stating that the process was both fun and rewarding. The project promoted critical dialogue within the group and was an opportunity for participants to discover something new about themselves and their subjectivity.

Secondly, this research's findings will contribute to the gap in the geographies of sexuality literature pertaining to the queer youth experience of their everyday space and subjectivity. It also has provided an understanding of queer subjectivity in the local context of Wellington.

Thirdly, the production of a zine and this research's conclusions will be given to InsideOUT to inform their future work. The zine will also be distributed to local libraries and made available to students and their schools as a resource that will hopefully stimulate change.

\subsection{Limitations and Future Considerations}

One limitation of this research was the time and monetary constraint placed upon the photovoice project that stopped the queer youth fully participating in the research design, implementation, and analysis stages. Although the participants were able to share their thoughts, agree and disagree with each other, and make decisions on safe and unsafe spaces in the focus group and interviews, the project was conceived of by me and ultimately, I carried out the final analysis and chose how to represent participants and their information in this thesis.

A further limitation of this research is that I was only able to recruit participants over sixteen years of age. This thesis was unable to give voice to younger youth and excludes experiences of space and subjectivity in what quite critical years for selfdiscovery and realisation. It is also foregrounds the voices of gender non-binary youth and those with 'questioning' sexualities as a result of the recruitment process. 
Several of the limitations of the research could be overcome with further research into the following areas:

Future research could explore the experiences of younger people, and people with other subjectivities under the queer label that were absent from this research, such as the perspectives of takatāpui, gay males, trans, asexual, and intersex subjectivities. It is also important that future research values and includes other intersecting subjectivities like culture, religion, age, and aspects like family relationships, whether they youth are out or not, and experiences with mental health.

Although this research and photovoice project does intend to disseminate the finished zine to InsideOUT and the wider community, future research or projects could work towards increasing awareness of heteronormativity with queer youth though avenues like policy, education, and campaigning. 


\section{REFERENCE LIST}

Advocates for Youth. (2016). Lesbian, Gay, Bisexual, and Transgender (LGBT) Youth in the Global South. United States of America. Retrieved from https://advocatesforyouth.org/resources/fact-sheets/lesbian-gay-bisexual-andtransgender-lgbt-youth-in-the-global-south/

Anderson, G. (2017). 'Why Can’t they Meet in Bars and Clubs like Normal People?': The Protective State and Bioregulating Gay Public Sex Spaces. Social \& Cultural Geography, 19(6), 1-21.

Banks, M. (2007). Using Visual Data in Qualitative Research. London, United Kingdom: SAGE Publications.

Batiste, D. P. (2013). “0 Feet Away”: The Queer Cartography of French Gay Men’s Geo-social Media Use. Anthropological Journal of European Cultures, 22(2), 111-132.

Bech, H. (1997). When Men Meet: Homosexuality and Modernity. Cambridge, United Kingdom: Polity Press.

Bell, D. (2009). Heteronormativity. In R. Kitchin \& N. Thrift (Eds.), International Encyclopedia of Human Geography (pp. 115-119). Oxford, United Kingdom: Elsevier.

Bell, D., Binnie, J., Cream, J., \& Valentine, G. (1994). All Hyped Up and No Place To Go. Gender, Place \& Culture, 1(1), 31-47.

Bell, D., \& Valentine, G. (1995). Mapping Desire: Geographies of Sexualities. London, United Kingdom; New York, NY: Routledge.

Blunt, A., \& Dowling, R. (2006). Home. London, United Kingdom: Taylor \& Francis. 
Bristow, J. (1989). Being Gay: Politics, Identity, Pleasure. New Formations, (9), 6181.

Brown, M. (2000). Closet Space Geographies of Metaphor from the Body to the Globe. London, United Kingdom: Routledge.

Browne, K. (2007). (Re) Making the Other, Heterosexualising Everyday Space. Environment and Planning A, 39(4), 996-1014.

Browne, K. (2009). Queer Theory/Queer Geographies. In R. Kitchin \& N. Thrift (Eds.), International Encyclopedia of Human Geography (pp. 39-45). Oxford, United Kingdom: Elsevier.

Browne, K., \& Nash, C. J. (2010). Queer Methods and Methodologies: An Introduction. In K. Browne \& C. J. Nash, Queer Methods and Methodologies: Intersecting Queer Theories and Social Science Research (pp. 1-23). London, United Kingdom: Ashgate.

Butler, J. (1990). Gender Trouble: Feminism and the Subversion of Identity. New York, NY: Routledge.

Butler, J. (1993). Bodies That Matter: On the Discursive Limits of "Sex.” New York, NY: Routledge.

Butler, J. (2008). Gender Trouble: Still Revolutionary or Obsolete? [Interview]. Cameron, J. (2005). Focussing on the Focus Group. In I. Hay (Ed.), Qualitative Research Methods in Human Geography (2nd ed., pp. 152-172). Oxford, United Kingdom: Oxford University Press.

Carlson, E., Engebretson, J., \& Chamberlain, R. (2006). Photovoice as a Social Process of Critical Consciousness. Qualitative Health Research, 16(6), 836852. 
Carroll, A., \& Mendos, L. (2017). State-Sponsored Homophobia 2017: A World Survey of Sexual Orientation Laws: Criminalisation, Protection and Recognition. Geneva, Switzerland: International Lesbian, Gay, Bisexual, Trans and Intersex Association. Retrieved from https://ilga.org/statesponsored-homophobia-report

Cavanagh, S. (2010). Queering Bathrooms: Gender, Sexuality, and the Hygienic Imagination. Toronto, Canada: University of Toronto Press.

Childress, H. (2005). Teenagers, Territory and the Appropriation of Space. Childhood: A Global Journal of Child Research, 11(2), 195-205.

Collins, D. (2009). Private/Public Divide. In R. Kitchin \& N. Thrift (Eds.), International Encyclopedia of Human Geography (pp. 437-441). Oxford, United Kingdom: Elsevier.

Coughlan, T. (2018, October 16). Gender Reassignment Surgery Cap Lifted. Newsroom. Retrieved from https://www.newsroom.co.nz/2018/10/15/277680?slug=genderreassignment-surgery-cap-lifted

Courpasson, D. (2017). The Politics of Everyday. Organization Studies, 38(6), 843859.

Cresswell, T. (2009). Place. In R. Kitchin \& N. Thrift (Eds.), International Encyclopedia of Human Geography (pp. 169-177). Oxford, United Kingdom: Elsevier.

Creswell, J. W. (2014). Research Design: Qualitative, Quantitative, and Mixed Methods Approaches (4th ed.). Thousand Oaks, CA: SAGE Publications. 
Desyllas, M. C., \& Sinclair, A. (2014). Zine-Making as a Pedagogical Tool for Transformative Learning in Social Work Education. Social Work Education, 33(3), 296-316.

Dilley, P. (1999). Queer Theory: Under Construction. International Journal of Qualitative Studies in Education, 12(5), 457-472.

Doan, P. (2007). Queers in the American City: Transgendered Perceptions of Urban Space. Gender, Place \& Culture, 14(1), 57-74.

Dowling, R. (2005). Power, Subjectivity, and Ethics in Qualitative Research. In I. Hay (Ed.), Qualitative Research Methods in Human Geography (2nd ed., pp. 26-39). Oxford, United Kingdom: Oxford University Press.

Duncan, N. (1996a). Renegotiating Gender and Sexuality in Public and Private Spaces. In N. Duncan (Ed.), Body Space: Destabilising Geographies of Gender and Sexuality (pp. 127-145). London, United Kingdom: Routledge.

Duncan, N. (1996b). Sexuality in Public and Private Spaces. In N. Duncan (Ed.), Body Space: Destabilising Geographies of Gender and Sexuality (pp. 127145). London, United Kingdom: Routledge.

Dunn, K. (2005). Interviewing. In I. Hay (Ed.), Qualitative Research Methods in Human Geography (2nd ed., pp. 101-138). Oxford, United Kingdom: Oxford University Press.

Evans, B. (2008). Geographies of Youth/Young People. Geography Compass, 2(5), $1659-1680$.

Fischer, H. (1977). Gay Semiotics. San Francisco, CA: NFS Press.

Foucault, M. (1970). The Order of Things: An Archaeology of the Human Sciences. London, United Kingdom: Tavistock. 
Foucault, M. (1972). The Archaeology of Knowledge. (S. Smith, Trans.). London, United Kingdom: Tavistock.

Foucault, M. (1979). Discipline and Punish: The Birth of the Prison (1st ed.). New York, NY: Pantheon Books.

Fox, C., \& Ore, T. (2010). (Un) Covering Normalized Gender and Race Subjectivities in LGBT “Safe Spaces.” Feminist Studies, 36(3), 629-649.

Fraser, V. (2010). Queer Closets and Rainbow Hyperlinks: The Construction and Constraint of Queer Subjectivities Online. Sexuality Research and Social Policy, 7(1), 30-36.

Freitag, M. (2013). A Queer Geography of a School: Landscapes of Safe(r) Spaces. Confero: Essays on Education, Philosophy and Politics, 1(2), 123-161.

Frith, H., \& Harcourt, D. (2005). Picture This: Using Photography to Explore Women’s Experience of Chemotherapy. Health Psychology Update, 14(3), 2-9.

Gagnier, R. (1991). Subjectivities: A History of Self-Representation in Britain, 18321920. Oxford, United Kingdom: Oxford University Press.

Giffney, N. (2009). Introduction. The “Q” Word. In M. O’Rourke \& N. Giffney, The Ashgate Research Companion to Queer Theory (pp. 1-13). Surrey, United Kingdom: Ashgate.

Gorman-Murray, A. (2007). Reconfiguring Domestic Values: Meanings of Home for Gay Men and Lesbians. Housing, Theory and Society, 24(3), 229-246.

Gorman-Murray, A. (2012). Queer Politics at Home: Gay Men's Management of the Public/Private Boundary. New Zealand Geographer, 68(2), 111-120. 
Gorman-Murray, A., \& McKinnon, S. (2015). Queer Geography. In J. D. Wright (Ed.), International Encyclopedia of the Social \& Behavioral Sciences (2nd ed., pp. 759-764). Oxford, United Kingdom: Elsevier.

Government Equalities Office. (2018). National LGBT Survey (No. GEO - RR001) (p. 304). Retrieved from www.gov.uk/government/consultations/nationallgbt-survey

Hall, D. E. (2004). Subjectivity. New York, NY; London, United Kingdom: Routledge.

Hartal, G. (2017). Fragile Subjectivities: Constructing Queer Safe Spaces. Social \& Cultural Geography, 19(8), 1-20.

Hartal, G., David, Y., \& Pascar, L. (2014). Safe Space. Mafte'akh - Lexical Review of Political Thought, (8), 93-120.

Harvey, D. (2002). Spaces of Capital: Towards a Critical Geography. New York, NY: Routledge.

Holtby, A., Klein, K., Cook, K., \& Travers, R. (2015). To Be Seen or Not To Be Seen: Photovoice, Queer and Trans Youth, and The Dilemma of Representation. Action Research, 13(4), 317-335.

Honeychurch, K. G. (1996). Researching Dissident Subjectivities: Queering the Grounds of Theory and Practice. Harvard Educational Review, 66(2), 339356.

Hubbard, P. (2005). Space/Place. In D. Sibley, P. Jackson, \& N. Washbourne (Eds.), Cultural Geography: A Critical Dictionary of Key Ideas (pp. 41-48). London, United Kingdom; New York, NY: I.B. Tauris.

Hubbard, P. (2008). Here, There, Everywhere: The Ubiquitous Geographies of Heteronormativity. Geography Compass, 2(3), 640-658. 
Hubbard, P., Kitchin, R., \& Valentine, G. (Eds.). (2004). Editor’s Introduction. In Key Thinkers on Space and Place. London, United Kingdom: Sage Publications.

Human Rights Campaign. (2018). LGBTQ Youth Report. Human Rights Campaign. Retrieved from https://www.hrc.org/resources/2018-lgbtq-youth-report/ Inness, S. A. (1997). The Lesbian Menace: Ideology, Identity, and the Representation of Lesbian Life. Boston, MA: University of Massachusetts Press.

Jagose, A. (1996). Queer Theory. Dunedin, New Zealand: Otago University Press. Johnson, C. (2002). Heteronormative Citizenship and the Politics of Passing. Sexualities, 5(3), 317-336.

Johnston, L. (2015). Sexuality and Space. In J. D. Wright (Ed.), International Encyclopedia of the Social \& Behavioral Sciences (2nd ed., pp. 808-812). Oxford, United Kingdom: Elsevier.

Johnston, L. (2019). Transforming Gender, Sex, and Place: Geographies of Gender Variance. Oxon, United Kingdom; New York, NY: Routledge. Johnston, L., \& Longhurst, R. (2010). Space, Place, and Sex: Geographies of Sexualities. Plymouth, United Kingdom: Rowman \& Littlefield. Kindon, S., Pain, R., \& Kesby, M. (2007). Participatory Action Research: Origins, Approaches and Methods. In S. Kindon, R. Pain, \& M. Kesby (Eds.), Participatory Action Research Approaches and Methods: Connecting People, Participation and Place. (pp. 9-18). London: Routledge. Kitchin, R. (2009). Space II. In International Encyclopedia of Human Geography (pp. 268-275). Oxford, United Kingdom: Elsevier. 
Knopp, L. (2007). From Lesbian and Gay to Queer Geographies: Past, Prospects and Possibilities. In K. Browne, J. Lim, \& G. Brown (Eds.), Geographies of Sexualities: Theory, Practices, and Politics (pp. 21-28). Hampshire, United Kingdom: Ashgate.

Lefebvre, H. (1991). Critique of Everyday Life. London, United Kingdom: Verso.

Lucassen, M., Clark, T., Moselen, E., \& Robinson, E. (2014). Youth'12 The Health and Wellbeing of Secondary School Students in New Zealand: Results for Young People Attracted to the Same Sex or Both Sexes (p. 45). Auckland, New Zealand: Adolescent Health Research Group. Retrieved from https://oro.open.ac.uk/43995/1/Same\%20Sex\%20Report_14NM.pdf

Massey, D. (1994). Space, Place and Gender. Minnesota, MN: University of Minnesota Press.

Massey, D. (1999). Power-Geometries and the Politics of Space-Time. Heidelberg, Germany: University of Heidelberg.

Massumi, B. (1992). A User's Guide to Capitalism and Schizophrenia: Deviations from Deleuze and Guattari. Cambridge, MA: MIT Press.

McBreen, K., Waitaha, K. M., \& Tahu, N. (2012). Ahunga Tikanga and Sexual Diversity. Te Tākupu, Te Wānanga o Raukawa, 21-35.

McDowell, L. (1995). Body Work: Heterosexual Gender Performances in City Workplaces. In D. Bell \& G. Valentine (Eds.), Mapping Desire: Geographies of Sexualities (pp. 75-95). London, United Kingdom; New York, NY: Routledge. 
McIntosh, P. (1988). White Privilege and Male Privilege: A Personal Account of Coming To See Correspondences through Work in Women’s Studies. Wellesley College Center for Research on Women. Retrieved from https://www.racialequitytools.org/resourcefiles/mcintosh.pdf

Mertens, D. (2008). Transformative Research and Evaluation. New York, NY: Guilford Press.

Mika, C. (2007). Locating the Lisp Gene. In J. Hutchings \& C. Aspin (Eds.), Sexuality and The Stories of Indigenous People (pp. 133-140). Wellington, New Zealand: Huia Publishers.

Nagar, R. (2002). Women's Theater and the Redefinitions of Public, Private, and Politics in North India. ACME: An International Journal for Critical Geographies, 1(1), 55-72.

Nash, C. (2011). Trans Experiences in Lesbian and Queer Space. The Canadian Geographer, 55(2), 192-207.

Nash, C. (2015). Queer Conversations. In K. Browne \& C. Nash (Eds.), Youth Cultures and Subcultures: Australian Perspectives (pp. 129-142). Surrey, United Kingdom: Ashgate.

Newell, F., \& Shams, L. (2007). New Insights into Multisensory Perception. Perception, 36(10), 1415-1417.

Nusser, S. P., \& Anacker, K. B. (2013). What Sexuality Is This Place? Building a Framework for Evaluating Sexualized Space: The Case of Kansas City, Missouri. Journal of Urban Affairs, 35(2), 173-193.

Nykiforuk, C. I. J., Vallianatos, H., \& Nieuwendyk, L. M. (2011). Photovoice as a Method for Revealing Community Perceptions of the Built and Social Environment. International Journal of Qualitative Methods, 10(2), 103-124. 
Penn, D. (1995). Queer: Theorizing Politics and History. Radical History Review, 62, 24-43.

Pink, S. (2011). A Multisensory Approach to Visual Methods. In E. Margolis \& L.

Pauwels, The SAGE Handbook of Visual Research Methods (pp. 601-614).

London, United Kingdom: SAGE Publications.

Potter, J., \& Wetherell, M. (1994). Analyzing Discourse. In A. Bryman \& R. G. Burgess (Eds.), Analyzing Qualitative Data (pp. 47-66). London, United Kingdom; New York, NY: Routledge.

Probyn, E. (1992). Technologizing The Self: A Future Anterior. In L. Grossberg, C. Nelson, \& P. Treichler (Eds.), Cultural Studies (pp. 501-511). New York, NY: Routledge.

Probyn, E. (1994). Lesbians in Space: Gender, Sex and the Structrue of Missing. Gender, Place and Culture, 2(1), 77-84.

Probyn, E. (2003). The Spatial Imperative of Subjectivity. In K. Anderson, M. Domosh, S. Pile, \& N. Thrift (Eds.), Handbook of Cultural Geography (pp. 290-299). London, United Kingdom: SAGE Publications.

Reavey, P., \& Johnson, K. (2017). Visual Approaches: Using and Interpreting Images. In C. Willig \& W. S. Rogers, The SAGE Handbook of Qualitative Research in Psychology (pp. 354-373). London, United Kingdom: SAGE Publications.

Revill, G. (2000). Music and the Politics of Sound: Nationalism, Citizenship, and Auditory Space. Environment and Planning D: Society and Space, 18(5), 597-613.

Richardson, D. (1998). Sexuality and Citizenship. Sociology, 32(1), 83-100. 
Rodó-de-Zárate, M. (2015). Young Lesbians Negotiating Public Space: An

Intersectional Approach through Places. Children’s Geographies, 13(4), 413-434.

Rogers, A. (2012). Geographies of the Performing Arts: Landscapes, Places and Cities. Geography Compass, 6(2), 60-75.

Rose, G. (1996). Teaching Visualised Geographies: Towards a Methodology for the Interpretation of Visual Materials. Journal of Geography in Higher Education, 20(3), 281-294.

Rose, G. (2001). Visual Methodologies: An Introduction to The Interpretation of Visual Materials. London, United Kingdom: SAGE Publications.

Rosenfeld, H., \& Noterman, E. (2014). Safe Space: Towards a Reconceptualization. Antipode, 46(5), 1346-1365.

Rushbrook, D. (2002). Cities, Queer Space, and the Cosmopolitan Tourist. GLQ: A Journal of Lesbian and Gay Studies, 8(1), 183-206.

Rutgers. (2016). The Photovoice Facilitator's Guide. Retrieved from https://www.rutgers.international/sites/rutgersorg/files/PDF/PhotoVoice_Ma nual_18042016.pdf

Saldanha, A. (2005). Trance and Visibility at Dawn: Racial Dynamics in Goa’s Rave Scene. Social \& Cultural Geography, 6(5), 707-721.

Schroeder, C. (2012). Making Space for Queer Youth: Adolescent and Adult Interactions in Toledo, Ohio. Gender, Place \& Culture, 19(5), 635-651.

Schroeder, C. (2015). Sanctuary or Prison: Queer Youth and the Family, Household and Home. Social \& Cultural Geography, 16(7), 1-15.

Sedgwick, E. K. (2008). Epistemology of the Closet. Berkeley, CA: University of California Press. 
Seidman, S. (1995). Deconstructing Queer Theory or the Under-Theorization of the Social and the Ethical. In L. Nicholson \& S. Seidman (Eds.), Social Postmodernism: Beyond Identity Politics (pp. 116-142). Cambridge, MA: Cambridge University Press.

Sibley, D. (1995). Geographies of Exclusion. London, United Kingdom: Routledge. Smyth, C. (1992). Lesbians Talk Queer Notions. London, United Kingdom: Scarlet. Spargo, T. (1999). Foucault and Queer theory. Cambridge, United Kingdom: Icon Books.

Stella, F. (2015). Carving Out Queer Space: In/visibility, Belonging and Resistance. In F. Stella (Ed.), Lesbian Lives in Soviet and Post-Soviet Russia: Post/Socialism and Gendered Sexualities (pp. 111-131). London, United Kingdom: Palgrave Macmillan UK.

Strack, R. W., Magill, C., \& McDonagh, K. (2004). Engaging Youth Through Photovoice. Health Promotion Practice, 5(1), 49-58.

Švab, A., \& Kuhar, R. (2014). The Transparent and Family Closets: Gay Men and Lesbians and their Families of Origin. Journal of GLBT Family Studies, 10(1-2), 15-35.

Taylor, J., \& Dwyer, A. (2015). Sexualities and Sensitivities: Queer(y)ing the Ethics of Youth Research in the Field. In S. Baker (Ed.), Youth Cultures and Subcultures : Australian Perspectives (pp. 241-251). Ashgate.

Taylor, Y. (2016). Making Space for Young Lesbians? Gendered Sites, Scripts and Sticking Points. In Y. Taylor (Ed.), Making Space for Queer-Identifying Religious Youth (pp. 77-92). London, United Kingdom: Palgrave Macmillan UK. 
Toomey, R. B., McGuire, J. K., \& Russell, S. T. (2012). Heteronormativity, School Climates, and Perceived Safety for Gender Nonconforming Peers. Journal of Adolescence, 35(1), 187-196.

Townsend, L. (2018). Rupe, Carmen Tione [Web page]. Retrieved from https://teara.govt.nz/en/biographies/6r6/rupe-carmen-tione

Tuan, Y.-F. (1979). Space and Place: Humanistic Perspective. In S. Gale \& G. Olsson (Eds.), Philosophy in Geography (pp. 387-427). Dordrecht, Netherlands: Springer.

Tuttleman, L. (2000). Presenting a Queer (Bath)House. In J. A. Boone, M. Dupuis, K. Quimby, M. Meeker, C. Sarver, D. Silverman, \& R. Weatherston (Eds.), Queer Frontiers: Millennial Geographies, Genders, and Generations (pp. 222-257). Madison, WI: University of Wisconsin Press.

Valentine, G. (1989). The Geography of Women’s Fear. Area, 21(4), 385-390.

Valentine, G. (1993). Negotiating and Managing Multiple Sexual Identities: Lesbian Time-Space Strategies. Transactions of the Institute of British Geographers, 18(2), 237-248.

Valentine, G. (1996). Renegotiating the “Heterosexual Street”: Lesbian Productions of Space. In N. Duncan (Ed.), Body Space: Destabilising Geographies of Gender and Sexuality (pp. 146-155). London, United Kingdom: Routledge.

Valentine, G. (2001). Social Geographies: Space and Society. New York, NY: Prentice Hall.

Valentine, G. (2002). Queer Bodies and the Production of Space. In D. Richardson \& S. Seidman (Eds.), Handbook of Lesbian and Gay Studies (pp. 145-160). London, United Kingdom: SAGE Publications. 
Valentine, G., \& Skelton, T. (2003). Finding Oneself, Losing Oneself: The Lesbian and Gay 'Scene’ as a Paradoxical Space. International Journal of Urban and Regional Research, 27(4), 849-866.

Valentine, G., Skelton, T., \& Chambers, D. (2005). Cool Places: An Introduction to Youth and Youth Cultures. In T. Skelton \& G. Valentine (Eds.), Cool Places: Geographies of Youth Cultures (pp. 1-32). London, United Kingdom: Routledge.

Vanderbeck, R. M., \& Johnson, J. (2000). “That’s the Only Place Where You Can Hang Out”: Urban Young People and the Space of the Mall. Urban Geography, 21(1), 5-25.

Waitt, G. (2010). Doing Foucauldian Discourse Analysis - Revealing Social Realities. In I. Hay (Ed.), Qualitative Research Methods in Human Geography (3rd ed.). Oxford, United Kingdom: Oxford University Press.

Wang, C. (2006). Youth Participation in Photovoice as a Strategy for Community Change. Journal of Community Practice, 14(1-2), 147-161.

Wang, C., \& Burris, M. A. (1994). Empowerment through Photo Novella: Portraits of Participation. Health Education Quarterly, 21(2), 171-186.

Wang, C., \& Burris, M. A. (1997). Photovoice: Concept, Methodology, and Use for Participatory Needs Assessment. Health Education \& Behavior, 24(3), 369387.

Warner, M. (1993). Fear of a Queer Planet: Queer Politics and Social Theory. Minneapolis, MN: University of Minnesota Press.

West, C., \& Zimmerman, D. (1990). Doing Gender. In J. Lorber \& S. A. Farrell (Eds.), The Social Construction of Gender (pp. 13-38). Thousand Oaks, CA: SAGE Publications. 
Youniss, J., \& Yates, M. (1997). Community Service and Social Responsibility in Youth. Chicago, IL: University of Chicago Press. 


\section{APPENDICIES}

\section{Appendix A - Photo Information Sheet}

\begin{tabular}{|c|c|}
\hline On a daily basis as a heterosexual and cisgender person.... & $\begin{array}{c}\text { Do I feel the same privilege } \\
\text { as a queer person? } \\
\text { Yes/No/Sometimes }\end{array}$ \\
\hline $\begin{array}{l}\text { I am pretty sure my friends, family, flat mates and work } \\
\text { colleagues will be comfortable with me being cis and } \\
\text { heterosexual. }\end{array}$ & \\
\hline $\begin{array}{l}\text { If I pick up a random magazine, or turn on the TV, I am sure } \\
\text { that my cis and heterosexual identity will be represented. }\end{array}$ & \\
\hline $\begin{array}{l}\text { When I talk to others about me being cis and heterosexual I } \\
\text { will NOT be accused of pushing my gender or sexual identity } \\
\text { onto others }\end{array}$ & \\
\hline $\begin{array}{l}\text { I could walk in public confidentially holding my partner’s } \\
\text { hand and NOT have people double-take or make comments }\end{array}$ & \\
\hline $\begin{array}{l}\text { People can use and describe my cis and heterosexual identity } \\
\text { to mean positive things ('Straight as an arrow', 'Standing up } \\
\text { straight') instead of demeaning terms ('eww that's gay', } \\
\text { 'man-up') }\end{array}$ & \\
\hline $\begin{array}{l}\text { I can go a month without saying 'I am straight' or 'I identify } \\
\text { as a male' }\end{array}$ & \\
\hline I can walk into a bar/café and feel like I can be myself & \\
\hline $\begin{array}{l}\text { I see art, posters and advertising that shows other cis } \\
\text { heterosexuals }\end{array}$ & \\
\hline $\begin{array}{l}\text { I can always call my partner 'boyfriend' or 'girlfriend' to } \\
\text { strangers }\end{array}$ & \\
\hline I am always called by my preferred pronouns & \\
\hline $\begin{array}{l}\text { I always identify other cis and heterosexual people as I walk } \\
\text { down the street }\end{array}$ & \\
\hline $\begin{array}{l}\text { I can walk into the bathroom without feeling like I don’t } \\
\text { belong }\end{array}$ & \\
\hline I can dress the way I want to everyday & \\
\hline I feel comfortable taking public transport & \\
\hline
\end{tabular}

Any more you can think of? 


\section{Appendix B - Photo Information Sheet}

The Queer-Space Photovoice project is your chance to share your experiences as queer youth as you go about your day-to-day lives and interact with different spaces. Here are some ideas to get you thinking about what pictures of spaces you should take:

- Remember the topic: What are your experiences as queer youth in different everyday spaces?

- Safe spaces: Make a collection of photos of different spaces where you feel comfortable, safe, and happy to be queer.

- Not so safe spaces: Make a collection of photos of different spaces where you feel scared, uncomfortable, and shy - like you need to be different - because you are queer.

- Think queer: Take pictures of specific spaces that make you think of your queer identity.

- Use your emotions as cue: You should take photos of spaces that you interact with quite often. As you walk, rest, play, relax and socialise during your day-to-day life, if you feel something in a space take a photo, then have a think later why you took a photo of that space. The photo of a space doesn't have show anything in particular, it can just be a picture of a space where you felt something. Just ask yourself why you feel this way.... 


\section{Appendix C - Health and Safety Information Sheet}

\section{Photographing people}

- Ask permission to take photos of people before you take them

- Only photograph people you know - we need to get the person to sign an 'Image release form' if you decide to pick the photo, so we need to be able to contact them after the picture is taken.

- Do not take pictures of people who are "in private", such as through a window into their home

- Ask yourself, "Would I mind if someone took a picture of me in this situation?”

- You do not need an Image release form if people are too small to be recognizable in the space- say a picture of Civic Square

\section{Representation}

- As you take photos, remember that you need to write a caption to display along with each photo so think about what are you trying to represent in the photo

- Using a camera gives the photographer a lot of power to create a message that is visually loaded with meaning; within the image is the photographer's values and message. Viewers will see these clearly in your photos. Therefore, it's important to represent the image and the places within the image in an accurate way

- Taking a photo of someone doing something risky or incriminating or taking a picture of a dangerous space would go against the values and goals of Photovoice and risk your safety

Safety

- Don't take any risks

- Don't go anywhere you wouldn't usually go, or do anything you wouldn't usually do

- Be aware of what's around you. When taking photos in the city, be street smart and stay away from roads and busy intersections - stop, look, listen

- If you feel unsafe, leave the space. Your safety is the most important thing 


\title{
Appendix D - Interview Guide
}

\author{
Welcome
}

Overall Experience

How did you find the photovoice experience?

\section{Generating Themes}

Layout photos and get the participants to start explaining their photo. Get the participant to start grouping the photos into themes. Make notes of the groupings by writing down the number assigned to each photo in each group.

\section{Safe Spaces}

What makes a space safe for you?

- People

- Location

- Is there ever a safe space?

What makes a space unsafe for you?

- People

- Location

- How can we make these spaces safer / Can we make these spaces safer?

Was it easier taking pictures of safe or unsafe spaces?

\section{Performance}

Do you ever feel like you have to change/hide your identity in certain spaces?

- What spaces are these?

- Do you feel like you have to perform to being cis-gendered/heterosexual?

How do you change/hide who you are in a space?

Why do you feel you have to alter how you act/look/perform?

\section{Representation}

Do you feel like your gender / sexual identity is represented in your everyday spaces? Why do you think this?

\section{Private/Public}

What is your experience as a queer youth like at home?

Do you feel differently being at home than out in public?

Why do you feel more/less comfortable being at home?

What makes public spaces different to being at home?

\section{School}

What is your experience as a queer youth like at school?

What aspects of the school spaces effect how you feel in them?

\section{Other people}

How do different people in these spaces

What aspects of the school spaces effect how you feel in that space? 


\section{Captioning}

Choose 7-10 photos, one or two from each theme. The criteria for picking is:

- $\quad$ The photo does not have to be the best looking, it is whatever photo has the better meanings portrayed in them.

- Does the photos you picked best show the overall themes of your photos?

Why did you choose this photo?

What is being portrayed in this photo?

What is it about this space that made you want to take this photo?

From these questions create a caption for your photos.

[If pushed for time create as many captions as possible then ask them to create the remaining ones at home]

Discuss that these photos will be used in the thesis - Sign Image Release Form

What information would you like attributed to your photos?

Name / Age / Pronouns / Gender / Sexual Identity 


\title{
Appendix E - Participant Information Sheet
}

\author{
TE WHARE WĀNAVGA O TE ŪPOKO O TE IKA A MĀUI

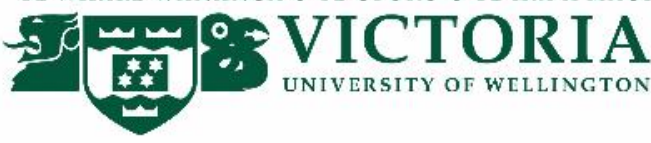 \\ Sexualised Spaces: Using Photovoice to Understand Queer Youths' experiences with
their Everyday Spaces
}

INFORMATION SHEET FOR PARTICIPANTS

You are invited to take part in this research. Please read this information before deciding whether or not to take part. If you decide to participate, thank you. If you decide not to participate, thank you for considering this request.

\section{Who am I?}

My name is Matthew Scott and I am a Master of Science student in Geography at Victoria University of Wellington. This research project is work towards my thesis.

\section{What is the aim of the project?}

This project aims to understand the experiences of self-identified queer youth as they interact with everyday spaces. It also aims to promote queer youth's experiences of space to the wider community to help foster positive queer experiences of space and place and highlight the influence of heterosexual norms. Participants will use photovoice to generate real-world experiences of space and place.

This research has been approved by the Victoria University of Wellington Human Ethics Committee 0000026050.

\section{What is Photovoice?}

Photovoice is a participatory photographic research method. It involves the participants of a study taking photographs that represent their individual perspectives and lived experiences. These pictures are then shared back with other participants and discussed to pull out connecting themes. Narratives or stories explaining the significance of the pictures will be attached to the photographs. A presentation or gallery exhibition will be produced to share the photos and experiences of the participants.

\section{How can you help?}

For the research to be beneficial to you, attendance is important at all parts of the project therefore you are committing to the following aspects of this project. Let Matt know if you cannot attend due to unavoidable events like illness.

Participants will meet three times with facilitators in audio and video recorded focus groups at a mutually agreed location. The first two focus groups will be around one hour long. There will then be a two-week gap in which you will take photographs of your perspectives and experiences that relate to, or illustrate the research topic: 'Space, Sexuality and Identity'. The third focus group will run for two hours after the photo-taking period and will involve the sharing of your photographs with the rest of the group. You will also be asked to meet 
with Matt for an hour audio-recorded interview prior to the last focus group to discuss your photos. Matt will ask question about your photos and experiences at the interview and focus groups.

After the interview and focus groups, participants can provide input into the creation of a presentation/gallery in Wellington or at your High School (this will be decided as a group). Here your photos can be displayed, with your permission, to showcase your work and the feelings and experiences self-identified queer youth have in different spaces.

The information shared during the focus groups will be confidential. That means after the focus group, you may not communicate anything that was shared to anyone else, including family members and close friends.

Participation is voluntary and you may refuse to participate or withdraw from the project at any time before $01 / 10 / 2018$ for any reason. If this occurs, you are free to choose between removing your contributions to the study or releasing them for use without your participation. Only my supervisor and I will have access to your photos, interview and focus group transcripts and summaries. The interview transcripts, recordings and summaries as well as your photos will be kept securely in locked filing cabinets and on password protected computers and will be destroyed on 04/03/2024.

\section{What will the project produce?}

The information from my research will be used in a thesis publication, future academic publications and presentations, a public exhibition and in a resource for InsideOUT. Your photos may be used with your permission in any of these outputs. You will be asked to sign a photo release form to allow Matt and his supervisor to publish your photos in the above outputs after you have taken and chosen your photos. You will have ownership over your photos, will be given proper acknowledgments and also receive a copy of your final photos.

You will have the option to have your identity made confidential in any or all of the research outputs. This means that the Matt will be aware of your identity, but your identity will not be revealed in any reports, presentations, or public documentation unless you wish it to be. If you don't want to use your own name, a pseudonym will replace your name, but your age, gender and sexual identity, and high school name will be published. You can change your mind on confidentiality at the third focus group and before the gallery if you want to.

Please note that because you will be participating in focus groups, your identity will not be confidential to the other members of the three focus group sessions. You should also be aware that in small projects like this, your identity might be obvious to others in your community or high school.

\section{If you accept this invitation, what are your rights as a research participant?}

You do not have to accept this invitation if you don't want to. If you do decide to participate, you have the right to:

- $\quad$ choose not to answer any question; 
- $\quad$ choose what photos are released for display and publication;

- $\quad$ ask for the recorder to be turned off at any time during the interview or focus groups;

- $\quad$ withdraw from the focus group while it is taking part, however it will not be possible to withdraw the information you have provided up to that point;

- $\quad$ ask any questions about the study at any time;

- $\quad$ receive a copy of your interview recordings;

- $\quad$ receive a copy of your interview transcripts;

- $\quad$ read over and comment on the transcripts of your interview;

- $\quad$ be able to read any reports of this research by emailing the researcher to request a copy.

- $\quad$ withdraw from the study before $01 / 10 / 2018$;

\section{If you have any questions or problems, who can you contact?}

If you have any questions, either now or in the future, please feel free to contact either:

\section{Matthew Scott}

MSc Geography Student

matt.scott@vuw.ac.nz

\section{Human Ethics Committee information}

\section{Sara Kindon}

Supervisor and A/Prof of Human Geography and Development Studies

School of Geography, Environment and Earth Sciences

(04) 4636194

sara.kindon@vuw.ac.nz

If you have any concerns about the ethical conduct of the research you may contact the

Victoria University HEC Convenor: Dr Judith Loveridge, email hec@vuw.ac.nz, phone (04) 4636028. 


\section{Appendix F - Participant Consent Form}

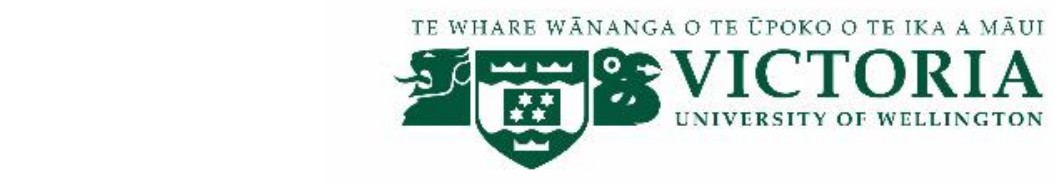

Sexualised Spaces: Using Photovoice to Understand Queer Youths’ experiences with their Everyday Spaces

\section{CONSENT TO PARTICIPATE IN PHOTO-VOICE, INTERVIEW AND FOCUS GROUPS}

This consent form will be held for five years.

Researcher: Matthew Scott, School of Geography, Environment and Earth Sciences, Victoria University of Wellington.

- I have read the Information Sheet and the project has been explained to me. My questions have been answered to my satisfaction. I understand that I can ask further questions at any time.

- $\quad$ I agree to take part in the photovoice project which would mean spending two weeks taking photos that represent my experience of the research topic.

- $\quad$ I am aware that I will be asked to participate in three audio and video recorded focus groups and one interview.

- I also agree to provide input into the creation of a presentation or gallery of the photos produced by myself and other participants

I understand that:

- I may withdraw from this study at any point before 01 October 2018, and any information that I have provided will be returned to me or destroyed, unless it is part of a focus group discussion. All identifiable information I have provided will be destroyed on 04 March 2024. If I remove myself from the study, identifiable data will be destroyed when the request is received.

- I I am agreeing to keep the information shared during the focus group confidential. I am aware that after the focus group, I must not communicate to anyone, including family members and close friends, any details about the focus group.

- $\quad$ I can withdraw from the focus group or interviews while they are in progress however it will not be possible to withdraw the information I have provided in the focus groups up to that point as it will be part of a discussion with other participants

- I understand that the results of this research will be used for thesis publication, future academic publications and presentations, a public exhibition and in a resource for InsideOUT. 
- $\quad$ I understand that the photographs I take will remain my intellectual property however I will grant the right of use to the researcher (Matthew Scott and his supervisor Sara Kindon) for thesis publication, future academic publications and presentations, a public exhibition and in a resource for InsideOUT by signing the 'Photo Release Form' after you have taken and selected your photos.

Please indicate:

- $\quad$ I consent to information, photos and quotes which I have given being attributed to me in any publications produced in this research. You can Yes No change your mind at the last focus group and before the gallery.

If no, my name will not be used in these publications, instead a pseudonym will be used. What pseudonym would you like to use:

- I would like a copy of the recording of my interview: Yes No

- I would like a copy of the transcript of my interview: Yes No

- I would like to receive a copy of the final thesis and have added my email Yes No address below.

Signature of participant:

Name of participant:

Date:

Contact details:

Email:

Cell: 